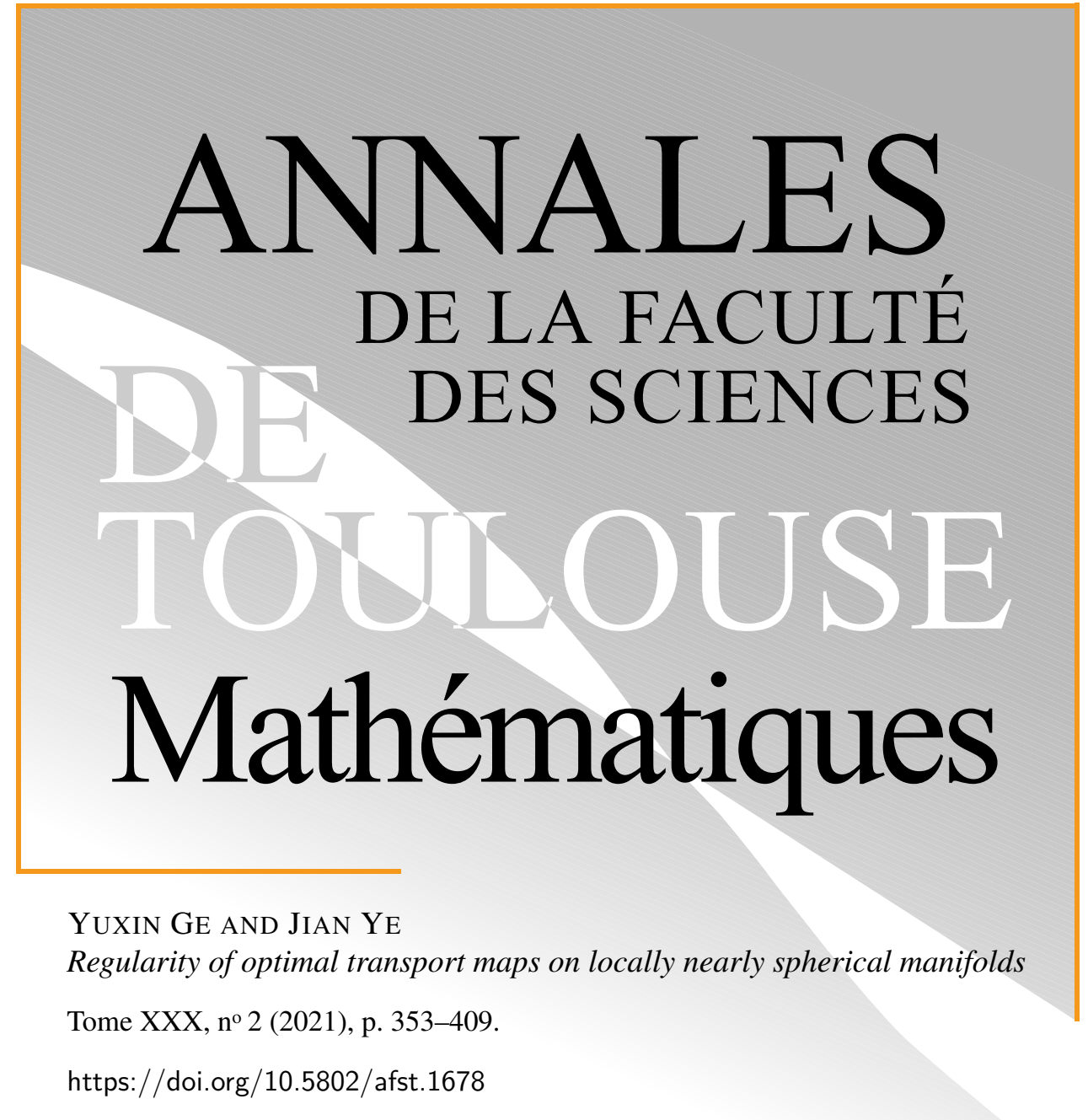

(c) Université Paul Sabatier, Toulouse, 2021.

L'accès aux articles de la revue «Annales de la faculté des sciences de Toulouse Mathématiques » (http://afst.centre-mersenne.org/) implique l'accord avec les conditions générales d'utilisation (http://afst.centre-mersenne.org/legal/). Les articles sont publiés sous la license CC-BY 4.0.

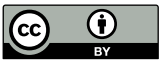

Publication membre du centre Mersenne pour l'édition scientifique ouverte MERSENNE http://www.centre-mersenne.org/ 


\title{
Regularity of optimal transport maps on locally nearly spherical manifolds
}

\author{
YUXIN GE ${ }^{(1)}$ AND JIAN YE ${ }^{(2)}$
}

\begin{abstract}
Given a compact connected $n$-dimensional Riemannian manifold, we investigate the smoothness of the optimal transport map between the smooth densities with respect to the squared Riemannian distance cost. The optimal map is characterized by $\exp (\operatorname{grad} u)$, where the potential function $u$ satisfies a MongeAmpère type equation. Delanoë [7] showed the smoothness of $u$ on the Riemannian surfaces when the scalar curvature is close to 1 in $C^{2}$ norm. In this work, we study the regularity issue on Riemannian manifolds with curvature sufficiently close to curvature of round sphere in $C^{2}$ norm in all dimensions and prove that the $\mathcal{C}$-curvature on such Riemannian manifolds satisfies an improved Ma-Trudinger-Wang condition and the Jacobian of the exponential map is positive. As a consequence, we imply the smoothness of the optimal transport map by the continuity method.
\end{abstract}

RÉSUMÉ. - Etant donné une variété riemannienne compacte connexe de dimension $n$, nous étudions la régularité de l'application du transport optimal entre les densités lisses par rapport au coût de la distance riemannienne au carré. L'application du transport optimal est caractérisée par $\exp (\operatorname{grad} u)$, où la fonction potentielle $u$ satisfait une équation de type Monge-Ampère. Delanoë [7] a montré la régularité de $u$ sur les surfaces riemanniennes lorsque la courbure scalaire est proche de 1 dans la norme $C^{2}$. Dans ce travail, nous étudions le problème de régularité sur les variétés riemanniennes avec courbure suffisamment proche de la courbure de la sphère usuelle dans la norme $C^{2}$ en toutes les dimensions et prouvons que la $\mathcal{C}$-courbure sur de telles variétés riemanniennes satisfait une condition Ma-Trudinger-Wang améliorée et le jacobien de l'application exponentielle est strictement positive. Par conséquent, nous impliquons la régularité de l'application du transport optimal par la méthode de continuité.

Keywords: regularity, optimal transport maps.

2020 Mathematics Subject Classification: 35R01, 53C21, 49N60.

(1) Institut de Mathématiques de Toulouse, Université Toulouse 3, 118, route de Narbonne, 31062 Toulouse Cedex, France — yge@math.univ-toulouse.fr

(2) School of Mathematical Sciences, University of Science and Technology of China, 96 Jinzhai Road, Baohe District, Hefei, Anhui, China — yejn573@mail.ustc.edu.cn The second author is supported by the scholarship from China Scholarship Council (CSC) under the Grant CSC NO.201506340070. 


\section{Introduction and main results}

\subsection{Background}

Let $(M, g)$ be a compact connected Riemannian manifold without boundary of dimension $n \geqslant 2$. For short, we call such $(M, g)$ as a closed Riemannian manifold. Let $d(\cdot, \cdot)$ be geodesic distance on $M$ and denote dvol the Riemannian volume form. We consider the optimal transportation problem on $M$ with the cost $c(\cdot, \cdot)=\frac{1}{2} d^{2}(\cdot, \cdot)$. The problem of optimal transportation is to find the most efficient strategy to transport an assigned mass distribution to another one. Precisely, let $\mu_{0}=\rho_{0}$ dvol and $\mu_{1}=\rho_{1}$ dvol be two positive Borel probability measures on $M$ with the density $\rho_{0}$ and $\rho_{1}$ with respect to the volume form dvol. The problem consists in minimizing the total cost functional

$$
\operatorname{Cost}(G)=\int_{M} c(x, G(x)) \mathrm{d} \mu_{0}
$$

among all Borel measurable maps $G: M \rightarrow M$ which push forward $\mu_{0}$ to $\mu_{1}$ in the sense that

$$
\mu_{1}(E)=\mu_{0}\left(G^{-1}(E)\right), \quad \forall E \subset M \text { Borel set. }
$$

The minimizers are called optimal transport maps. This problem is first posed by Monge [34] in 1781 with the Euclidean distance cost $c(x, y)=|x-y|$. One and a half centuries later, Kantorovich [20] reduced the problem to an infinite dimensional linear program. For the squared Euclidean distance cost $c(x, y)=\frac{1}{2}|x-y|^{2}$, Brenier [1] showed the existence and uniqueness of the optimal transport map which can be characterized as the gradient of some convex function. McCann [32] developed Brenier's theory on Riemannian manifolds. He showed the optimal transport map is unique and takes the form $G(m)=\exp _{m}(\nabla u(m))$ where $u$ is some $c$-convex function, that is, $\forall x \in M, u(x)=\sup _{y \in M}(-c(x, y)-v(y))$ for some function $v$ on $M$. Such function $u$ is called the potential function of the optimal transport map $G$. The aim of this paper is to show the smoothness of the optimal transport maps $G$, or equivalently, the smoothness of the optimal transport potential $u$. Ma-Trudinger-Wang [31] introduced for the first time the MTW tensor which is crucial in the study of the regularity theory of the optimal transport maps. Later on, Kim-McCann [22] interpreted the MTW tensor as a curvature tensor of some pseudo-Riemannian metric. There are a lot of developments in the past decade for example $[5,7,8,9,11,13,15,17,24,26,27,28,30,35]$ etc. For more references, see the book of Villani [36].

We recall the definition of the $\mathcal{C}$-curvature. Given $m \in M$, denote by Cut $_{m} \subset M$ the cut locus of $M$ at $m$. The closed subset Cut of $T M$ is 
defined by

$$
\text { Cut }=\left\{(m, \nu) \in M \times T_{m} M, \exp _{m} \nu \in \mathrm{Cut}_{m}\right\} .
$$

We consider the open connected component of $T M \backslash$ Cut containing the zero section and denote it by

$$
\text { NoCut }=\{(m, \nu), \forall t \in[0,1] \text { and }(m, t \nu) \notin \mathrm{Cut}\} .
$$

Given $(m, \nu) \in \operatorname{NoCut},(\xi, \eta) \in T_{m} M \times T_{m} M$, we define the $\mathcal{C}$-curvature by

$$
\mathcal{C}(m, \nu)(\xi, \eta)=-\left.\left.\frac{3}{2} \frac{\partial^{2}}{\partial s^{2}}\right|_{s=0} \frac{\partial^{2}}{\partial t^{2}}\right|_{t=0} c\left(\exp _{m} t \xi, \exp _{m}(\nu+s \eta)\right) .
$$

For the more intrinsic geometric interpretation, we use the aforementioned pseudo-Riemannian metric $h$ on $M \times M$ and set Sect $_{h}$ for its sectional curvature tensor viewed as a field of quadratic forms on $\bigwedge^{2} T(M \times M)$, for each $(m, \bar{m}) \in M \times M \backslash \mathrm{Cut}_{M}$ with $\mathrm{Cut}_{M}=\exp (\mathrm{Cut})$ the cut locus of $M$ and each $(\xi, \bar{\xi}) \in T_{m} M \times T_{\bar{m}} M$, the associated cross-curvature is defined in $[22]$ :

$$
\operatorname{cross}_{(m, \bar{m})}(\xi, \bar{\xi}):=\operatorname{Sect}_{h}[(\xi \oplus 0) \wedge(0 \oplus \bar{\xi})] .
$$

Kim and McCann [22] observed that it must vanish for some choice of $(\xi, \bar{\xi})$. Trudinger et al. noted [31, p. 164] that one identically recovers $\frac{1}{2} \operatorname{cross}_{(m, \bar{m})}(\xi, \bar{\xi})$ at $\bar{m}=\exp _{m}(V)$ with $(m, V) \in$ NoCut and $\bar{\xi}=$ $d\left(\exp _{m}\right)(V)(\nu)$, by calculating the quantity:

$$
\mathcal{C}(m, V)(\xi, \nu):=-\frac{D^{2}}{d \lambda^{2}}[A(m, V+\lambda \nu)(\xi)]_{\lambda=0}
$$

where $A(m, V)(\xi):=\nabla d\left[p \rightarrow c\left(p, \exp _{m}(V)\right)\right]_{p=m}(\xi, \xi)$ with $\nabla$ the LeviCivita connection of the Riemannian metric $g$ and where $D$ stands for the canonical flat connection of $T_{m} M$.

When $\nu=0$, Loeper [28] observed that the $\mathcal{C}$-curvature is just the sectional curvature. In fact, it follows from the Taylor expansion for the geodesic distance [33, p. 5] that

$$
\begin{aligned}
d^{2}\left(\exp _{m} t \xi, \exp _{m} s \eta\right)=|\xi|_{m}^{2} t^{2}- & 2 g_{m}(\xi, \eta) t s+|\eta|_{m}^{2} s^{2} \\
& -\frac{1}{3} R_{m}(\xi, \eta, \xi, \eta) t^{2} s^{2}+o\left(\left(t^{2}+s^{2}\right)^{2}\right) .
\end{aligned}
$$

Thus we see $\mathcal{C}(m, 0)(\xi, \eta)=R_{m}(\xi, \eta, \xi, \eta)$. More generally, we have the asymptotic expansion [24] for the $\mathcal{C}$-curvature

$\mathcal{C}(m, \nu)(\xi, \eta)=R_{m}(\xi, \eta, \xi, \eta)+\frac{1}{2}\left(\nabla_{\eta} R\right)(\xi, \nu, \xi, \eta)+\frac{1}{4}\left(\nabla_{\nu} R\right)(\xi, \eta, \xi, \eta)+o(|\nu|)$. 
We introduce several curvature conditions $[23,31,35]$

(i) A3S condition is satisfied if there exists some $\kappa_{0}>0$ such that $\mathcal{C}(m, \nu)(\xi, \eta) \geqslant \kappa_{0}|\xi|^{2}|\eta|^{2}, \forall(m, \nu) \in$ NoCut, $\forall(\xi, \eta) \in T_{m} M \times T_{m} M$ with $\langle\xi, \eta\rangle=0$

(ii) A3W condition is satisfied if $\mathcal{C}(m, \nu)(\xi, \eta) \geqslant 0, \forall(m, \nu) \in$ NoCut, $\forall$ $(\xi, \eta) \in T_{m} M \times T_{m} M$ with $\langle\xi, \eta\rangle=0$

(iii) the non-negatively c-curved (or NNCC) condition is satisfied if $\mathcal{C}(m, \nu)(\xi, \eta) \geqslant 0, \forall(m, \nu) \in$ NoCut,$\forall(\xi, \eta) \in T_{m} M \times T_{m} M$

(iv) the almost-positively c-curved (or APCC) condition is satisfied if NNCC condition is satisfied and $\mathcal{C}(m, \nu)(\xi, \eta)=0$ if and only if the span of the vectors $(\nu, \xi, \eta)$ has dimension at most 1.

The A3S condition is satisfied on the round sphere [29] and on nearly spherical manifolds $[8,14,17,30]$. The NNCC condition is stable under Riemannian products, unlike A3W condition. Each of the above conditions is stable under Riemannian submersion [23]. The APCC condition holds on spheres [23] (see also $[8,14,29]$ ), on projective spaces $\mathbb{C P}^{k}$ and $\mathbb{H P}^{k}[23]$ and on positively curved Riemannian locally symmetric space [10]. The APCC condition is satisfied on surfaces close to 2-sphere [9]. On the other hand, the A3W condition can imply nonnegative sectional curvature[28], but the inverse is not true $[16,21,30]$.

For the regularity issue, the $\mathrm{A} 3 \mathrm{~W}$ condition is necessary for the continuity of the optimal transport map [28] and also sufficient under some suitable assumptions [35]. There are many works related to the $C^{1}$ regularity of potential $u$ (or continuity of optimal transport maps) for all measures $\mu_{0}, \mu_{1}$ (possibly not smooth). For the instance, see references [14, 15, 16, 17, 23] etc. Here we are interested in the high order regularity on closed manifolds. Such regularity result holds on flat manifolds [5], on spheres [29], on complexe or quaternionic projective spaces $[7,13,23]$ (see also [27]), on product of spheres [12,13,23], on nearly spherical manifolds with topology [8,30] and on 2 dimensional simply connected manifolds or positively curved Riemannian locally symmetric spaces [7].

\subsection{Main results and outline of the paper}

Let us recall some notations (see $[2,3,4,18])$. Let $X, Y, Z, W$ be smooth vector fields on $M$. The (3,1)-type Riemann curvature tensor of the Riemaniann manifold $(M, g)$ is defined by

$$
R(X, Y) Z=\nabla_{X} \nabla_{Y} Z-\nabla_{Y} \nabla_{X} Z-\nabla_{[X, Y]} Z
$$


where $\nabla$ denotes the Levi-Civita connection of $g$. We set Riem for the associated (4,0)-type Riemann curvature tensor ${ }^{(1)}$, that is.

$$
\operatorname{Riem}(X, Y, Z, W)=\langle R(Z, W) Y, X\rangle
$$

Here, we adopt the Einstein summation convention over repeated indexes. In a local coordinate system $\left\{x^{1}, \ldots, x^{n}\right\}$, the components of Riemann curvature tensor are given by $R\left(\frac{\partial}{\partial x^{j}}, \frac{\partial}{\partial x^{k}}\right) \frac{\partial}{\partial x^{i}}=R_{i j k}^{l} \frac{\partial}{\partial x^{l}}$ and $R_{i j k l}=g_{i p} R_{j k l}^{p}$ respectively. The Ricci tensor is obtained by the contraction $\operatorname{Ric}_{i j}=g^{k l} R_{i k j l}$ and the scalar curvature by Scal $=g^{i j} \operatorname{Ric}_{i j}$. The Riemannian metric induces norms on all the tensor bundles. More precisely, the squared norm of $(r, s)$ tensor field $T$ in the coordinate system $x=\left(x^{1}, \ldots, x^{n}\right)$ is given by

$$
|T|^{2}=g_{i_{1} k_{1}} \ldots g_{i_{s} k_{s}} g^{j_{1} l_{1}} \ldots g^{j_{r} l_{r}} T_{j_{1} \ldots j_{r}}^{i_{1} \ldots i_{s}} T_{l_{1} \ldots l_{r}}^{k_{1} \ldots k_{s}},
$$

Let $K: \operatorname{Gr}_{2}(M) \rightarrow \mathbb{R}$ be the sectional curvature defined on the Grassmann bundle of tangent 2-planes (see [7]). We always assume the sectional curvatures satisfy

$$
\min _{\operatorname{Gr}_{2}(M)} K=1 .
$$

and we define for the Riemann curvature tensor when $n \geqslant 3$

$$
\varepsilon:=\left\|\operatorname{Riem}-\frac{\text { Scal }}{2 n(n-1)} g \otimes g\right\|_{C^{2}(M, g)}
$$

Here the Kulkarni-Nomizu product $₫$ is defined as follows: given two 2covariant tensors $h$ and $k$, the Kulkarni-Nomizu product is a 4 -covariant tensor, determined by

$$
\begin{aligned}
(h \otimes k)\left(X_{1}, X_{2}, X_{3}, X_{4}\right):= & h\left(X_{1}, X_{3}\right) k\left(X_{2}, X_{4}\right)+k\left(X_{1}, X_{3}\right) h\left(X_{2}, X_{4}\right) \\
& -h\left(X_{1}, X_{4}\right) k\left(X_{2}, X_{3}\right)-k\left(X_{1}, X_{4}\right) h\left(X_{2}, X_{3}\right),
\end{aligned}
$$

where $X_{j}$ for $1 \leqslant j \leqslant 4$ are tangent vectors. If necessary, we could identify the contravariant tensor and the covariant one via the Riemannian metric $g$. In two dimension, the tensor on the right hand identically vanishes and we replace the quantity on the right hand in (1.4) by

$$
\varepsilon:=\|K-1\|_{C^{2}(M, g)}
$$

While the dimension $n \geqslant 3$, the quantity (1.4) vanishes if and only if $(M, g)$ is space form. It follows from [8] that we consider an equivalent term to (1.4) when $n \geqslant 3$ or (1.5) when $n=2$

$$
\bar{\varepsilon}:=\left\|\operatorname{Riem}-\frac{1}{2} g \otimes g\right\|_{C^{2}(M, g)} .
$$

(1) We use $g(\cdot, \cdot)$ and $\langle\cdot, \cdot\rangle$ interchangeably. 
It is known on the round spheres $\mathbb{S}^{n}$ that for $\nu \neq 0$

$$
\overline{\mathcal{A}}(m, \nu)(\xi)=|\xi|_{m}^{2}-\left(1-|\nu|_{m} \cot |\nu|_{m}\right)\left(|\xi|_{m}^{2}-g_{m}\left(\xi, \frac{\nu}{|\nu|_{m}}\right)^{2}\right)
$$

and we can calculate the $\mathcal{C}$-curvature

$$
\overline{\mathcal{C}}(m, \nu)(\xi, \eta)=-\left.\frac{3}{2} \frac{\mathrm{d}^{2}}{\mathrm{~d} s^{2}}\right|_{s=0} \overline{\mathcal{A}}(m, \nu+s \eta)(\xi) .
$$

It follows from [23] that the APCC condition holds on spheres $\mathbb{S}^{n}$. Our first main result concerns about the stability of the APCC condition.

TheOREm 1.1. - Let $(M, g)$ be a closed n-dimensional Riemannian manifold satisfying (1.3). Then there exists some universal positive constants $\varepsilon_{0}, \kappa_{0}$ such that if $\varepsilon<\varepsilon_{0}$, that is, when $n \geqslant 3$

$$
\| \text { Riem }-\frac{\text { Scal }}{2 n(n-1)} g \otimes g \|_{C^{2}(M, g)}<\varepsilon_{0}
$$

when $n=2$

$$
\|K-1\|_{C^{2}(M, g)}<\varepsilon_{0}
$$

Then for all $(m, \nu) \in$ NoCut and all tangent vectors $\xi, \eta$ in $T_{m} M$

$$
\mathcal{C}(m, \nu)(\xi, \eta) \geqslant \kappa_{0}\left(|\xi \wedge \eta|_{m}^{2}+|\xi|_{m}^{2}|\eta \wedge \nu|_{m}^{2}+|\xi \wedge \nu|_{m}^{2}|\eta|_{m}^{2}\right),
$$

where $|\xi \wedge \eta|_{m}^{2}=|\xi|_{m}^{2}|\eta|_{m}^{2}-g_{m}(\xi, \eta)^{2},|\eta \wedge \nu|_{m}^{2}=|\eta|_{m}^{2}|\nu|_{m}^{2}-g_{m}(\eta, \nu)^{2}$, $|\xi \wedge \nu|_{m}^{2}=|\xi|_{m}^{2}|\nu|_{m}^{2}-g_{m}(\xi, \nu)^{2}$.

Since the NNCC condition is stable for the Riemannian products, a direct consequence of the above theorem can be read as the following.

Corollary 1.2. - Assume $M$ and $N$ satisfy the assumptions as in Theorem 1.1. Then the A3W condition holds on the product manifold $M \times N$.

Once we prove the A3S condition, we could study the regularity of the optimal transport maps. For this purpose, we use the PDE setting. If the potential function $u$ is $C^{2}$, it satisfies the following fully nonlinear PDE

$$
\operatorname{det}\left(\operatorname{Hess}^{(c)}(u)(x)\right)=\mathcal{B}\left(x, \nabla_{x} u\right),
$$

where

and

$$
\operatorname{Hess}^{(c)}(u)(x):=[\nabla \mathrm{d} c(\cdot, q)]_{\left[x, \exp _{x}(\nabla u(x))\right]}+\nabla \mathrm{d} u(x),
$$

$$
\mathcal{B}\left(x, \nabla_{x} u\right):=\frac{\rho_{0}(x)}{\rho_{1}(G(x)) \operatorname{det}\left(\partial_{p} \exp _{x}(p)\left(x, \nabla_{x} u\right)\right)}
$$

An observation due to Delanoë [7, Lemma 3.5] is the stay away property, that is, if the potential $u$ is $C^{2}$, then for all point $m \in M$ the image point $G(m)$ of the optimal transport map does not meet the cut locus $\mathrm{Cut}_{m}$. This 
is some kind of the first order estimate for the potential $u$. With the help of Theorem 1.1, we prove the following regularity result.

TheOREM 1.3. - Let $(M, g)$ be a closed n-dimensional Riemannian manifold satisfying (1.3). Then there exists some universal positive constant $\bar{\varepsilon}_{1}$ such that if when $n \geqslant 3$

$$
\| \text { Riem }-\frac{\text { Scal }}{2 n(n-1)} g \oplus g \|_{C^{2}(M, g)}<\bar{\varepsilon}_{1}
$$

when $n=2$

$$
\|K-1\|_{C^{2}(M, g)}<\bar{\varepsilon}_{1}
$$

then for all given ( $\left.\rho_{0} \mathrm{dvol}, \rho_{1} \mathrm{dvol}\right)$ of $C^{k, \alpha}$ positive Borel probability measures on $M$ with $k \geqslant 2$ and $\alpha \in(0,1)$, the potential function of the optimal transport map is $C^{k+2, \alpha}$.

We use the continuity method to get the result. For this purpose, we need to establish the suitable estimates à priori. The main difficulties come from two parts: on one hand, we need to deduce the suitable $C^{2}$ estimates. This is solved by Ma-Trudinger-Wang [31] under the suitable positivity of $\mathcal{C}$-curvature. Thus, it is done with the help of result in Theorem 1.1; on the other hand, the optimal transport map $G$ needs to avoid the cut locus, that is, for all $m \in M, G(m)$ is uniformly away from the cut-locus of $m$. For this aim, we choose a suitable new test function to obtain the maximum principle. Delanöe [6] communicates kindly to us that he has obtained some stability result on A3S condition on the homogenous manifolds with positive sectional curvature and proved smoothness result on such manifolds.

The paper is organized as follows. Section 1 presents the main results of the paper. Section 2 is devoted to recall some notions of Riemannian geometry. In Section 3 we prove Theorem 1.1, that is, the $\mathcal{C}$-curvature satisfies APCC condition. The proof relies on a careful analysis together with the perturbative arguments comparing to the constant curvature case. It is divided into three cases: near the origin, the intermediate case and near the focalization. The asymptotic expansion of the $\mathcal{C}$-curvature gives the improved MTW condition near the origin. In the intermediate case, the fact that the improved MTW condition holds on the sphere gives rise to the improved MTW condition on $M$. We adapt the method [17] to verify improved MTW condition near the cut-locus. The last section is devoted to the proof of Theorem 1.3 by continuity method. We collect some known results and prove some technical results related to Jocobi fields in Appendix. 


\section{Preliminaries}

In this section, we collecte some notions in riemannian geometry $[2,3,4$, 18] (see also $[8,36]$ ).

\subsection{Basic notations and conventions}

Recall a fact that the tensor $g \oslash g$ is parallel, i.e.

$$
\nabla(g \oplus g)=0 .
$$

By the condition (1.3) and taking trace of the Riemann curvature tensor one and two times we get the following relations

$$
\|\operatorname{Ric}-(n-1) g\|_{C^{2}(M, g)} \simeq \varepsilon, \| \text { Scal }-n(n-1) \|_{C^{2}(M, g)} \simeq \varepsilon .
$$

The $(3,1)$-form of $\frac{1}{2} g \otimes g$ is denoted by $\bar{R}$, i.e.

$$
\bar{R}(X, Y) Z=\langle Y, Z\rangle X-\langle X, Z\rangle Y
$$

which is also the curvature tensor on the sphere $\mathbb{S}^{n}$. The components of $\bar{R}$ are given by $\bar{R}\left(\frac{\partial}{\partial x^{j}}, \frac{\partial}{\partial x^{k}}\right) \frac{\partial}{\partial x^{i}}=\bar{R}_{i j k}^{l} \frac{\partial}{\partial x^{l}}$.

By Bonnet Myers theorem [4], the normalization (1.3) implies $(M, g)$ is compact and there is conjugate point along every geodesic. Moreover, there is cut point along every geodesic [2]. Given $m \in M, \forall \nu \in T_{m} M$ with $|\nu|_{m}=1$, let $t_{C}(m, \nu)$ be the distance from point $m$ to the cut point of $m$ along the geodesic $\exp _{m}(t \nu)$, i.e.

$$
t_{C}(m, \nu)=\sup \left\{t \geqslant 0:\left.\exp _{m}(s \nu)\right|_{0 \leqslant s \leqslant t} \text { is a minimizing geodesic }\right\} .
$$

The injectivity domain at $m$ is denoted by $I(m)$, i.e.

$$
I(m)=\left\{t \nu: 0 \leqslant t<t_{C}(m, \nu), \nu \in T_{m} M \backslash\{0\}\right\} .
$$

The focal time $t_{F}(m, \nu)$ is defined by

$$
t_{F}(m, \nu)=\inf \left\{t \geqslant 0: \exp _{m}(t \nu) \text { is conjugate to } m\right\} .
$$

We recall that the cut time is smaller than the focal time, the injectivity domain is an open subset contains the origin in $T_{m} M$ and star-shaped with respect to origin. Moreover, $M=\exp _{m}(I(m)) \bigsqcup \mathrm{Cut}_{m}$, where $\bigsqcup$ means disjoint union. The exponential map $\exp _{m}: I(m) \rightarrow M \backslash \mathrm{Cut}_{m}$ is a diffeomorphism. We denote by $\exp _{m}^{-1}(y)$ all the velocities $\nu \in T_{m} M$ such that the geodesic $\left.\exp _{m}(s \nu)\right|_{0 \leqslant s \leqslant 1}$ is minimizing and $\exp _{m} \nu=y$.

The geometry of injectivity domain is complicated. But on some special manifolds they have special geometric properties. 
We state $\frac{1}{2} d^{2}(\cdot, \cdot)$ is smooth in $M \times M \backslash \mathrm{Cut}_{M}$. For any $y \notin \mathrm{Cut}_{m}$, the Gauss lemma implies that

$$
\nabla_{m} \frac{d^{2}}{2}(y, m)=-\exp _{m}^{-1} y .
$$

Given a real smooth function $u$ defined on $M$, the Hessian of $u$ at $m$ is given by the linear operator from $T_{m} M$ to $T_{m} M$ defined

$$
\forall \xi \in T_{m} M, \nabla_{m}^{2} u(\xi):=\nabla_{\xi}(\operatorname{grad} u) .
$$

It is easy to see that the Hessian is a self adjoint and can be calculated as follows

$$
\left\langle\nabla_{m}^{2} u(\xi), \xi\right\rangle=\left.\frac{\mathrm{d}^{2}}{\mathrm{~d} s^{2}}\right|_{s=0} u(\gamma(s)),
$$

where $\gamma$ is a geodesic such that $\gamma(0)=m$ and $\dot{\gamma}(0)=\xi$, where ${ }^{\circ}$ is the derivative with respect to the real variable $s$.

\subsection{Jacobi fields}

Definition 2.1. - Given $m \in M$ and $\nu \in T_{m} M \backslash\{0\}$, let $\left\{E_{1}, E_{2}, \ldots, E_{n}\right\}$ be an orthonormal basis of $T_{m} M$ with $E_{1}=\nu /|\nu|_{m}$. Let $\gamma(\cdot)$ be a geodesic with initial point $m$ and initial velocity $\nu$ and $\left\{e_{1}, e_{2}, \ldots, e_{n}\right\}$ be the parallel transport of $\left\{E_{1}, E_{2}, \ldots, E_{n}\right\}$ along $\gamma$. We define $J_{0}(m, \nu, t), J_{1}(m, \nu, t)$ as the matrix valued solutions of the second order equation

$$
\left\{\begin{array}{l}
\ddot{J}_{a}+R J_{a}=0, a=0,1, \\
J_{0}(m, \nu, 0)=0, \dot{J}_{0}(m, \nu, 0)=I_{n}, \\
J_{1}(m, \nu, 0)=I_{n}, \dot{J}_{1}(m, \nu, 0)=0 .
\end{array}\right.
$$

where the elements of $R$ are given by

$$
R_{i j}(t)=\left\langle R\left(e_{i}(t), \dot{\gamma}(t)\right) \dot{\gamma}(t), e_{j}(t)\right\rangle .
$$

As same as on the sphere, we define $\overline{J_{a}}(m, \nu, t)$ as the matrix-valued of the second order equation

$$
\ddot{\overline{J_{a}}}+\bar{R} \overline{J_{a}}=0, \quad a=0,1,
$$

with the initial condition $\bar{J}_{0}(m, \nu, 0)=0, \dot{\overline{J_{0}}}(m, \nu, 0)=I_{n}$ and $\bar{J}_{1}(m, \nu, 0)=$ $I_{n}, \dot{\overline{J_{1}}}(m, \nu, 0)=0$. The elements of $\bar{R}$ are given by

$$
\bar{R}_{i j}(m, \nu, t)=\left\langle\bar{R}\left(e_{i}(t), \dot{\gamma}(t)\right) \dot{\gamma}(t), e_{j}(t)\right\rangle .
$$


It is easy to see that $\bar{J}_{0}$ and $\bar{J}_{1}$ in the orthonormal basis $\left\{e_{1}, e_{2}, \ldots, e_{n}\right\}$ are given respectively by

$$
\bar{J}_{0}=\left[\begin{array}{cc}
t & 0 \\
0 & \frac{\sin (|\nu| t)}{|\nu|} I_{n-1}
\end{array}\right], \quad \bar{J}_{1}=\left[\begin{array}{cc}
1 & 0 \\
0 & \cos (|\nu| t) I_{n-1}
\end{array}\right] .
$$

From the homogeneity of a geodesic (see[2, p. 64]), we get the homogeneity of the Jacobi fields $\lambda J_{0}(m, \lambda \nu, t)=J_{0}(m, \nu, \lambda t), J_{1}(m, \lambda \nu, t)=$ $J_{1}(m, \nu, \lambda t), \forall \lambda>0$. For $t \in[0,1]$, we extended $J_{a}$ by continuity at $\nu=0$ by $J_{1}(m, 0, t)=t I_{n}$ and $J_{1}(m, 0, t)=I_{n}$. For simplicity, the Jacobi fields $J_{a}(m, \nu, t)$ are abbreviated to $J_{a}(t)$ unless otherwise specified. By the definition of conjugate points, the matrix $J_{0}(t)$ is invertible for $\forall t \in\left(0, t_{F}(m, \nu)\right)$. Moreover, the continuity of the Jacobi fields implies [36] $\operatorname{det} J_{0}(t)>0$ for $\forall t \in\left(0, t_{F}(m, \nu)\right)$. We first state the fundamental formula which will be used in Section 3 to calculate the $\mathcal{C}$-curvature, that is, the hessian of the squared distance can be expressed in terms of Jacobi fields and we have the representation formula for the inhomogeneous Jacobi equations. We recall some results in $[17,36]$.

Proposition 2.2. - Under the above assumptions, we have

(a) Given $\nu \in T_{m} M \backslash\{0\}$, let $J(t)$ be the Jacobi field along the geodesic $\exp _{m}(t \nu)$ determined by the conditions $J(0)=\xi, J(1)=0$ and $J(t) \neq 0$ for all $0<t<1$. Then $J(t)=-J_{0}(t) J_{0}^{-1}(1) J_{1}(1)(\xi)+$ $J_{1}(t)(\xi)$

(b) For $t \in\left[0, t_{F}(m, \nu)\right)$, let $S(m, \nu, t)$ be the linear operator from $T_{m} M$ to $T_{m} M$ whose matrix in the orthonormal basis $\left\{E_{1}, E_{2}, \ldots, E_{n}\right\}$ is given by $t J_{0}(t)^{-1} J_{1}(t)$. Then the linear operator $S(m, \nu, t): T_{m} M \rightarrow$ $T_{m} M$ is self adjoint. Moreover, if $\nu \in I(m)$, then for $\forall \xi \in T_{m} M$,

$$
\left\langle\nabla_{m}^{2} c\left(\cdot, \exp _{m} \nu\right)(\xi), \xi\right\rangle=\langle S(m, \nu, 1)(\xi), \xi\rangle .
$$

(c) (Representation formula) The solution of the matrix valued inhomogeneous Jacobi equation

$$
\ddot{J}(t)+R(t) J(t)=B(t)
$$

is given by the formula

$$
J(t)=J_{0}(t) \dot{J}(0)+J_{1}(t) J(0)+J_{0}(t) \int_{0}^{t} J_{1}^{*} B \mathrm{~d} s-J_{1}(t) \int_{0}^{t} J_{0}^{*} B \mathrm{~d} s,
$$

where $J_{a}^{*}$ is the transpose (or adjoint) of the matrix $J_{a}$ for $a=0,1$.

Remark 2.3. -

(1) (Homogeneity) From the homogeneity of the Jacobi fields, we have $S(m, \lambda \nu, t)=S(m, \nu, \lambda t)$ for $\lambda>0$. Then we can extended $S$ by continuity at $\nu=0$ by $S(m, 0, t)=I_{n}$. 
(2) The linear operator $S(m, \nu, t)$ has explicit formula on space forms [25], for instance, on the round sphere

$$
\mathbb{S}^{n}, \bar{S}(m, \nu, t)(\xi)=\xi-(1-t|\nu| \cot (t|\nu|))\left(\xi-\left\langle\xi, \frac{\nu}{|\nu|}\right\rangle \frac{\nu}{|\nu|}\right) .
$$

(3) Given some $C^{2}$ real valued function $f: I \subset \mathbb{R} \rightarrow \mathbb{R}$ defined on some interval $I$, we consider some ODE

$$
\ddot{f}+f=\phi,
$$

where ${ }^{-}$is the derivative with respect to the real variable. Hence, it is known that the representation formula holds

$$
\begin{aligned}
f(t)=f(0) \cos t+ & \dot{f}(0) \sin t \\
& +\sin t \int_{0}^{t} \phi(s) \cos s \mathrm{~d} s-\cos t \int_{0}^{t} \phi(s) \sin s \mathrm{~d} s .
\end{aligned}
$$

\subsection{Fermi coordinate system}

In this work, we use extensively the Fermi chart in the calculus. Here we recall the definition and some results in [8].

Definition 2.4 (Fermi coordinate system). - Let $(M, g)$ be a n-dimensional Riemannian manifold. Given $\left(m_{0}, v_{0}\right) \in$ NoCut with $v_{0} \neq 0$, let $I=$ $\left[0,\left|v_{0}\right|\right] \subset \mathbb{R}$ be a compact interval. Let $\gamma: I \rightarrow M$ such that $\gamma(t)=$ $\exp _{m_{0}}\left(t v_{0} /\left|v_{0}\right|\right)$ be a geodesic and $\left\{e_{1}(t), e_{2}(t), \ldots, e_{n}(t)\right\}$ be a parallel orthonormal moving frame of vector fields along the geodesic $\gamma$ with $e_{1}(t)=$ $\frac{\dot{\gamma}(t)}{|\dot{\gamma}(t)|}$. The Fermi coordinate system $\left\{x^{1}, \ldots, x^{n}\right\}$ are defined by

$$
\begin{aligned}
x^{1}\left(\exp _{\gamma(t)}\left(\sum_{\beta=2}^{n} \lambda^{\beta} e_{\beta}(t)\right)\right) & =\frac{t}{|\dot{\gamma}(t)|}, \\
x^{\alpha}\left(\exp _{\gamma(t)} \sum_{\beta=2}^{n} \lambda^{\beta} e_{\beta}(t)\right) & =\lambda^{\alpha}, \quad 2 \leqslant \alpha \leqslant n, t \in I .
\end{aligned}
$$

where $\lambda^{\beta}$ are sufficiently small so that the exponential maps are defined.

The Fermi coordinate system is generalization of the normal coordinate system. To see this, along the axis we have

$$
\forall x^{1} \in I, \forall i, j, k \in\{1,2, \ldots, n\}, g_{i j}\left(x^{1}, 0\right)=\delta_{i j}, \partial_{k} g_{i j}\left(x^{1}, 0\right)=0,
$$

where $\delta$ is Kronecker symbol. In the following, the Latin indices run over $1, \ldots, n$ and the Greek indices run over $2, \ldots, n$. We calculate higher order derivatives of the metric and Christoffel symbols in such chart. 
Lemma 2.5. - The following identities hold on the axis

$$
\begin{aligned}
\partial_{i j}^{2} g_{11} & =-2 R_{1 i 1 j}, \quad \partial_{\alpha \beta}^{2} g_{1 \mu}=-\frac{2}{3}\left(R_{\alpha 1 \beta \mu}+R_{\alpha \mu \beta 1}\right), \\
\partial_{\alpha \beta}^{2} g_{\rho \mu} & =-\frac{1}{3}\left(R_{\beta \rho \alpha \mu}+R_{\beta \mu \alpha \rho}\right), \\
\partial_{k} \Gamma_{1 j}^{i} & =R_{j k 1}^{i}, \quad \partial_{\alpha} \Gamma_{\beta \mu}^{i}=\frac{1}{3}\left(R_{\beta \alpha \mu}^{i}+R_{\mu \alpha \beta}^{i}\right), \\
\partial_{\alpha \beta}^{2} \Gamma_{11}^{i} & =\nabla_{1} R_{\beta \alpha 1}^{i}+\nabla_{\alpha} R_{1 \beta 1}^{i}, \\
\partial_{\alpha \beta}^{2} \Gamma_{1 \mu}^{1} & =\frac{1}{3}\left(\nabla_{1} R_{\beta \alpha \mu}^{1}-\nabla_{1} R_{\mu \beta \alpha}^{1}\right)-\nabla_{\alpha} R_{\mu 1 \beta}^{1}, \\
\partial_{\alpha \beta}^{2} \Gamma_{1 \mu}^{\rho} & =\frac{1}{2}\left(\nabla_{\alpha} R_{\mu \beta 1}^{\rho}+\nabla_{\beta} R_{\mu \alpha 1}^{\rho}\right)+\frac{1}{6}\left(\nabla_{1} R_{\alpha \beta \mu}^{\rho}+\nabla_{1} R_{\beta \alpha \mu}^{\rho}\right) .
\end{aligned}
$$

Proof. - All identities are proved in [8, Lemma 2] except (2.13). However, its proof is same. We leave the detail for the readers.

\section{Proof of Theorem 1.1}

We give first the expression of the $\mathcal{C}$-curvature. We adapt the presentation in [17]. The proof of theorem relies on a careful analysis together with the perturbative arguments comparing to the constant curvature case.

\section{1. $\mathcal{C}$-curvature calculation in dimension $n$}

In this subsection we calculate the $\mathcal{C}$-curvature. Fix $m_{0} \in M, \nu_{0} \in$ $I\left(m_{0}\right) \backslash\{0\}$ and $(\xi, \eta) \in T_{m_{0}} M \times T_{m_{0}} M$. Since the $\mathcal{C}$-curvature is homogeneous with degree 2 in both $\xi$ and $\eta$, it suffices to assume that $|\xi|_{m_{0}}=$ $|\eta|_{m_{0}}=1$. Fix an orthonormal basis $\left\{E_{1}, E_{2}, \ldots, E_{n}\right\}$ of the tangent space $T_{m_{0}} M$ so that $\nu_{0}=\left|\nu_{0}\right|_{m_{0}} E_{1}, \xi=\xi_{1} E_{1}+\xi_{2} E_{2}+\xi_{3} E_{3}, \eta=\eta_{1} E_{1}+\eta_{2} E_{2}$ and identify the tangent vectors at $m_{0}$ with their coordinates in this basis. Then the metric at the point $m_{0}$ is given by the canonical scalar product of $\mathbb{R}^{n}$. We denote $\tau=\left|\nu_{0}\right|$. It will be implicitly understood throughout the calculations that the inner product and the Riemann curvature are evaluated at the point $m_{0}$.

Combining (1.2) and (2.8), we have

$$
\begin{aligned}
\mathcal{C}\left(m_{0}, \nu_{0}\right)(\xi, \eta) & =-\left.\frac{3}{2} \frac{\mathrm{d}^{2}}{\mathrm{~d} s^{2}}\right|_{s=0}\left\langle\nabla_{m_{0}}^{2} c\left(\cdot, \exp _{m_{0}}\left(\nu_{0}+s \eta\right)\right)(\xi), \xi\right\rangle_{m_{0}} \\
& =-\left.\frac{3}{2} \frac{\mathrm{d}^{2}}{\mathrm{~d} s^{2}}\right|_{s=0}\left\langle S\left(m_{0}, \nu_{0}+s \eta, 1\right)(\xi), \xi\right\rangle_{m_{0}} .
\end{aligned}
$$


For any $s \in \mathbb{R}$ small enough, we can write $\nu_{0}+s \eta=t(s)\left(\cos \theta E_{1}+\sin \theta E_{2}\right)$, where

$$
t(s)=\left|\nu_{0}+s \eta\right|_{m_{0}}, \theta(s)=\tan ^{-1}\left(\frac{s \eta_{2}}{\tau+s \eta_{1}}\right) .
$$

Together with Remark 2.3, it follows that

$$
\mathcal{C}\left(m_{0}, \nu_{0}\right)(\xi, \eta)=-\left.\frac{3}{2} \frac{\mathrm{d}^{2}}{\mathrm{~d} s^{2}}\right|_{s=0}\left\langle S\left(m_{0}, \cos \theta(s) E_{1}+\sin \theta(s) E_{2}, t(s)\right)(\xi), \xi\right\rangle_{m_{0}} .
$$

We give some notations now. Let $\gamma_{\theta}(t)$ be the geodesic with initial point $m_{0}$ and initial velocity $\cos \theta E_{1}+\sin \theta E_{2}$. For $|\theta|$ small, let $\left\{e_{1}(\theta, t), e_{2}(\theta, t)\right.$, $\left.\ldots, e_{n}(\theta, t)\right\}$ be the parallel transport along the geodesic $\gamma_{\theta}(t)$ with $e_{1}(\theta, 0)=$ $\cos \theta E_{1}+\sin \theta E_{2}, e_{2}(\theta, 0)=-\sin \theta E_{1}+\cos \theta E_{2}, e_{i}(\theta, 0)=E_{i}$ for $i \geqslant 3$. Let $J_{0}(\theta, t), J_{1}(\theta, t)$ be the solutions of the Jacobi equation (2.5) and $R(\theta, t)$ the curvature matrix by (2.6) along the geodesic $\gamma_{\theta}(t)$. The matrix of $S\left(m_{0}, e_{1}(\theta, 0), t\right)$ in the orthonormal basis $\left\{e_{1}(\theta, 0), e_{2}(\theta, 0), \ldots, e_{n}(\theta, 0)\right\}$ is given by

$$
S(\theta, t)=t J_{0}(\theta, t)^{-1} J_{1}(\theta, t)
$$

Let

$$
Q(\theta)=\left[\begin{array}{ccc}
\cos \theta & \sin \theta & \\
-\sin \theta & \cos \theta & \\
& & I_{n-2}
\end{array}\right] .
$$

Then the matrix of $S\left(m_{0}, e_{1}(\theta, 0), t\right)$ in the orthonormal basis $\left\{E_{1}, E_{2}\right.$, $\left.\ldots, E_{n}\right\}$ is $Q(\theta)^{T} S(\theta, t) Q(\theta)$, i.e.

$$
\left\langle S\left(m_{0}, e_{1}(\theta, 0), t\right)(\xi), \xi\right\rangle=\langle S(\theta, t) Q(\theta) \xi, Q(\theta) \xi\rangle .
$$

Here, the dot stands for the derivative with respect to $t$ and the prime for the derivative with respect to $\theta$.

Differentiating (3.1) once and twice with respect to $s$ successively, we have

$$
\begin{aligned}
& \frac{\mathrm{d}}{\mathrm{d} s}\langle\left.S\left(m_{0}, e_{1}(\theta, 0), t\right)(\xi), \xi\right\rangle=\left[\left\langle S^{\prime} Q \xi, Q \xi\right\rangle+2\left\langle S Q \xi, Q^{\prime} \xi\right\rangle\right] \frac{\mathrm{d} \theta}{\mathrm{d} s}+\langle\dot{S} Q \xi, Q \xi\rangle \frac{\mathrm{d} t}{\mathrm{~d} s}, \\
& \frac{\mathrm{d}^{2}}{\mathrm{~d} s^{2}}\left\langle S\left(m_{0}, e_{1}(\theta, 0), t\right)(\xi), \xi\right\rangle \\
&=\left[\left\langle S^{\prime \prime} Q \xi, Q \xi\right\rangle+4\left\langle S^{\prime} Q \xi, Q^{\prime} \xi\right\rangle+2\left\langle S Q^{\prime} \xi, Q^{\prime} \xi\right\rangle+2\left\langle S Q \xi, Q^{\prime \prime} \xi\right\rangle\right]\left(\frac{\mathrm{d} \theta}{\mathrm{d} s}\right)^{2} \\
& \quad+\left[2\left\langle\dot{S}^{\prime} Q \xi, Q \xi\right\rangle+4\left\langle\dot{S} Q \xi, Q^{\prime} \xi\right\rangle\right] \frac{\mathrm{d} t}{\mathrm{~d} s} \frac{\mathrm{d} \theta}{\mathrm{d} s}+\langle\ddot{S} Q \xi, Q \xi\rangle\left(\frac{\mathrm{d} t}{\mathrm{~d} s}\right)^{2} \\
& \quad+\left[\left\langle S^{\prime} Q \xi, Q \xi\right\rangle+2\left\langle S Q \xi, Q^{\prime} \xi\right\rangle\right] \frac{\mathrm{d}^{2} \theta}{\mathrm{d} s^{2}}+\langle\dot{S} Q \xi, Q \xi\rangle \frac{\mathrm{d}^{2} t}{\mathrm{~d} s^{2}} .
\end{aligned}
$$


The direct computations lead to

$$
\begin{array}{ll}
\frac{\mathrm{d} t}{\mathrm{~d} s}=\frac{s\left(\eta_{1}^{2}+\eta_{2}^{2}\right)+\tau \eta_{1}}{\left|\nu_{0}+s \eta\right|}, & \frac{\mathrm{d}^{2} t}{\mathrm{~d} s^{2}}=\frac{\tau^{2} \eta_{2}^{2}}{\left|\nu_{0}+s \eta\right|^{3}}, \\
\frac{\mathrm{d} \theta}{\mathrm{d} s}=\frac{\tau \eta_{2}}{s^{2}\left(\eta_{1}^{2}+\eta_{2}^{2}\right)+2 s \tau \eta_{1}+\tau^{2}}, & \frac{\mathrm{d}^{2} \theta}{\mathrm{d} s^{2}}=-\frac{2 \tau \eta_{2}\left[s\left(\eta_{1}^{2}+\eta_{2}^{2}\right)+\tau \eta_{1}\right]}{\left[s^{2}\left(\eta_{1}^{2}+\eta_{2}^{2}\right)+2 s \tau \eta_{1}+\tau^{2}\right]^{2}},
\end{array}
$$

so that at $s=0$, we have

$$
t=\tau, \frac{\mathrm{d} t}{\mathrm{~d} s}=\eta_{1}, \frac{\mathrm{d}^{2} t}{\mathrm{~d} s^{2}}=\frac{\eta_{2}^{2}}{\tau}, \quad \theta=0, \frac{\mathrm{d} \theta}{\mathrm{d} s}=\frac{\eta_{2}}{\tau}, \frac{\mathrm{d}^{2} \theta}{\mathrm{d} s^{2}}=-\frac{2 \eta_{1} \eta_{2}}{\tau^{2}} .
$$

Writing

$$
P \xi=\left(\xi_{1}, \xi_{2}, 0, \ldots, 0\right)^{T}, \quad P^{\perp} \xi=\left(\xi_{2},-\xi_{1}, 0, \ldots, 0\right)^{T},
$$

then at $s=0$, we have

$$
Q \xi=\xi, \quad Q^{\prime} \xi=P^{\perp} \xi, \quad Q^{\prime \prime} \xi=-P \xi .
$$

Gathering (3.2), (3.3) and (3.4), we obtain

$$
\begin{aligned}
& \left.\frac{\mathrm{d}^{2}}{\mathrm{~d} s^{2}}\right|_{s=0}\left\langle S\left(m_{0}, e_{1}(\theta, 0), t\right)(\xi), \xi\right\rangle \\
& =\left[\left\langle S^{\prime \prime} \xi, \xi\right\rangle+4\left\langle S^{\prime} \xi, P^{\perp} \xi\right\rangle+2\left\langle S P^{\perp} \xi, P^{\perp} \xi\right\rangle-2\langle S \xi, P \xi\rangle\right] \frac{\eta_{2}^{2}}{\tau^{2}} \\
& \quad+\left[2\left\langle\dot{S}^{\prime} \xi, \xi\right\rangle+4\left\langle\dot{S} \xi, P^{\perp} \xi\right\rangle\right] \frac{\eta_{1} \eta_{2}}{\tau}+\langle\ddot{S} \xi, \xi\rangle \eta_{1}^{2} \\
& +\left[\left\langle S^{\prime} \xi, \xi\right\rangle+2\left\langle S \xi, P^{\perp} \xi\right\rangle\right]\left(-\frac{2 \eta_{1} \eta_{2}}{\tau^{2}}\right)+\langle\dot{S} \xi, \xi\rangle \frac{\eta_{2}^{2}}{\tau} \\
& =\langle\ddot{S} \xi, \xi\rangle \eta_{1}^{2}+\left[\frac{2}{\tau}\left\langle\dot{S}^{\prime} \xi, \xi\right\rangle+\frac{4}{\tau}\left\langle\dot{S} \xi, P^{\perp} \xi\right\rangle-\frac{2}{\tau^{2}}\left\langle S^{\prime} \xi, \xi\right\rangle-\frac{4}{\tau^{2}}\left\langle S \xi, P^{\perp} \xi\right\rangle\right] \eta_{1} \eta_{2} \\
& +\left[\frac{1}{\tau^{2}}\left\langle S^{\prime \prime} \xi, \xi\right\rangle+\frac{1}{\tau}\langle\dot{S} \xi, \xi\rangle+\frac{4}{\tau^{2}}\left\langle S^{\prime} \xi, P^{\perp} \xi\right\rangle+\frac{2}{\tau^{2}}\left\langle S P^{\perp} \xi, P^{\perp} \xi\right\rangle-\frac{2}{\tau^{2}}\langle S \xi, P \xi\rangle\right] \eta_{2}^{2}
\end{aligned}
$$

which yields

$$
\begin{aligned}
\mathcal{C}( & \left.m_{0}, \nu_{0}\right)(\xi, \eta) \\
= & -\frac{3}{2}\langle\ddot{S} \xi, \xi\rangle \eta_{1}^{2} \\
& +3\left[-\frac{1}{\tau}\left\langle\dot{S}^{\prime} \xi, \xi\right\rangle-\frac{2}{\tau}\left\langle\dot{S} \xi, P^{\perp} \xi\right\rangle+\frac{1}{\tau^{2}}\left\langle S^{\prime} \xi, \xi\right\rangle+\frac{2}{\tau^{2}}\left\langle S \xi, P^{\perp} \xi\right\rangle\right] \eta_{1} \eta_{2} \\
& +\frac{3}{2}\left[-\frac{1}{\tau^{2}}\left\langle S^{\prime \prime} \xi, \xi\right\rangle-\frac{1}{\tau}\langle\dot{S} \xi, \xi\rangle-\frac{4}{\tau^{2}}\left\langle S^{\prime} \xi, P^{\perp} \xi\right\rangle\right. \\
& \left.\quad-\frac{2}{\tau^{2}}\left\langle S P^{\perp} \xi, P^{\perp} \xi\right\rangle+\frac{2}{\tau^{2}}\langle S \xi, P \xi\rangle\right] \eta_{2}^{2} \\
= & a_{11}\left(m_{0}, \nu_{0}, \xi\right) \eta_{1}^{2}+a_{12}\left(m_{0}, \nu_{0}, \xi\right) \eta_{1} \eta_{2}+a_{22}\left(m_{0}, \nu_{0}, \xi\right) \eta_{2}^{2},
\end{aligned}
$$


where

$$
\begin{aligned}
a_{11}\left(m_{0}, \nu_{0}, \xi\right) & :=-\frac{3}{2}\langle\ddot{S} \xi, \xi\rangle \\
a_{12}\left(m_{0}, \nu_{0}, \xi\right) & :=3\left[-\frac{1}{\tau}\left\langle\dot{S}^{\prime} \xi, \xi\right\rangle-\frac{2}{\tau}\left\langle\dot{S} \xi, P^{\perp} \xi\right\rangle+\frac{1}{\tau^{2}}\left\langle S^{\prime} \xi, \xi\right\rangle+\frac{2}{\tau^{2}}\left\langle S \xi, P^{\perp} \xi\right\rangle\right] \\
a_{22}\left(m_{0}, \nu_{0}, \xi\right):=\frac{3}{2}\left[-\frac{1}{\tau^{2}}\left\langle S^{\prime \prime} \xi, \xi\right\rangle-\frac{1}{\tau}\langle\dot{S} \xi, \xi\rangle\right. & \left.\quad-\frac{4}{\tau^{2}}\left\langle S^{\prime} \xi, P^{\perp} \xi\right\rangle-\frac{2}{\tau^{2}}\left\langle S P^{\perp} \xi, P^{\perp} \xi\right\rangle+\frac{2}{\tau^{2}}\langle S \xi, P \xi\rangle\right]
\end{aligned}
$$

\subsection{Proof of Theorem 1.1 and Corollary 1.2}

The proof of Theorem 1.1 is divided into three cases: near the origin, the intermediate case and near the focalization. The asymptotic behaviour gives the improved MTW condition near the origin. In the intermediate case, we use the similar strategy in [8]: the fact that the improved MTW condition holds on the sphere gives rise to the improved MTW condition on $M$. We adapt the method [17] to verify improved MTW condition near the cut-locus.

\subsubsection{Asymptotic behaviour near the origin}

With the help of the above preparations, we can obtain our main result in this subsection as follows.

THEOREM 3.1. - Under the same assumptions as in Theorem 1.1, there exist some universal positive constants $\widetilde{\varepsilon}_{0}, \delta_{1}, \kappa_{1}$ such that if $\varepsilon<\widetilde{\varepsilon}_{0}$, then for all $\left(m_{0}, \nu_{0}\right) \in$ NoCut with $\tau=\left|\nu_{0}\right|<\delta_{1}$ and all tangent vectors $\xi, \eta$ in $T_{m_{0}} M$

$$
\mathcal{C}\left(m_{0}, \nu_{0}\right)(\xi, \eta) \geqslant \kappa_{1}\left(|\xi \wedge \eta|_{m_{0}}^{2}+|\xi|_{m_{0}}^{2}\left|\eta \wedge \nu_{0}\right|_{m_{0}}^{2}+\left|\xi \wedge \nu_{0}\right|_{m_{0}}^{2}|\eta|_{m_{0}}^{2}\right) \text {. }
$$

Proof. - We deduce first the expansion of the $\mathcal{C}$-curvature near the origin. For this purpose, by (B.25)(B.26)(B.27) below, we have

$$
\begin{aligned}
& \mathcal{C}\left(m_{0}, \nu_{0}\right)(\xi, \eta) \\
& =\left[R\left(\xi, E_{1}, \xi, E_{1}\right)+\frac{3}{4} \tau\left(\nabla_{1} R_{1212} \xi_{2}^{2}+2 \nabla_{1} R_{1213} \xi_{2} \xi_{3}+\nabla_{1} R_{1313} \xi_{3}^{2}\right)\right. \\
& \left.\quad+\frac{2}{5} \tau^{2}\left(\xi_{2}^{2}+\xi_{3}^{2}\right)+O\left(\varepsilon \tau^{2}+\tau^{4}\right)\left(\xi_{2}^{2}+\xi_{3}^{2}\right)\right] \eta_{1}^{2}
\end{aligned}
$$




$$
\begin{aligned}
& +\left\{2 R\left(\xi, E_{1}, \xi, E_{2}\right)+\tau\left[\frac{1}{2} \nabla_{2} R_{1212} \xi_{2}^{2}+\left(\nabla_{1} R_{1223}+\nabla_{2} R_{1213}\right) \xi_{2} \xi_{3}\right.\right. \\
& \left.+\left(\nabla_{1} R_{1323}+\frac{1}{2} \nabla_{2} R_{1313}\right) \xi_{3}^{2}-\nabla_{1} R_{1212} \xi_{1} \xi_{2}-\nabla_{1} R_{1213} \xi_{1} \xi_{3}\right] \\
& \left.-\frac{2}{5} \tau^{2} \xi_{1} \xi_{2}+O\left(\varepsilon \tau^{2}+\tau^{3}\right)\left(\xi_{2}^{2}+\xi_{3}^{2}+\xi_{1} \xi_{2}+\xi_{1} \xi_{3}\right)\right\} \eta_{1} \eta_{2} \\
& +\left\{R\left(\xi, E_{2}, \xi, E_{2}\right)+\tau\left[\frac{1}{4} \nabla_{1} R_{1212} \xi_{1}^{2}-\frac{1}{2} \nabla_{2} R_{1212} \xi_{1} \xi_{2}\right.\right. \\
& -\frac{1}{2}\left(\nabla_{1} R_{1223}+\nabla_{2} R_{1213}\right) \xi_{1} \xi_{3}+\frac{1}{2} \nabla_{2} R_{1223} \xi_{2} \xi_{3} \\
& \left.+\left(\frac{1}{4} \nabla_{1} R_{2323}+\frac{1}{2} \nabla_{2} R_{1323}\right) \xi_{3}^{2}\right] \\
& \left.+\frac{\tau^{2}}{15}\left(\xi_{1}^{2}+\xi_{2}^{2}+2 \xi_{3}^{2}\right)+O\left(\varepsilon \tau^{2}+\tau^{4}\right)\right\} \eta_{2}^{2} \\
& =R\left(\xi, E_{1}, \xi, E_{1}\right) \eta_{1}^{2}+2 R\left(\xi, E_{1}, \xi, E_{2}\right) \eta_{1} \eta_{2}+R\left(\xi, E_{2}, \xi, E_{2}\right) \eta_{2}^{2} \\
& +\frac{3}{4} \tau\left(\nabla_{1} R_{1212} \xi_{2}^{2}+2 \nabla_{1} R_{1213} \xi_{2} \xi_{3}+\nabla_{1} R_{1313} \xi_{3}^{2}\right) \eta_{1}^{2} \\
& +\tau\left[\frac{1}{2} \nabla_{2} R_{1212} \xi_{2}^{2}+\left(\nabla_{1} R_{1223}+\nabla_{2} R_{1213}\right) \xi_{2} \xi_{3}\right. \\
& \left.+\left(\nabla_{1} R_{1323}+\frac{1}{2} \nabla_{2} R_{1313}\right) \xi_{3}^{2}-\nabla_{1} R_{1212} \xi_{1} \xi_{2}-\nabla_{1} R_{1213} \xi_{1} \xi_{3}\right] \eta_{1} \eta_{2} \\
& +\tau\left[\frac{1}{4} \nabla_{1} R_{1212} \xi_{1}^{2}-\frac{1}{2} \nabla_{2} R_{1212} \xi_{1} \xi_{2}-\frac{1}{2}\left(\nabla_{1} R_{1223}+\nabla_{2} R_{1213}\right) \xi_{1} \xi_{3}\right. \\
& \left.+\frac{1}{2} \nabla_{2} R_{1223} \xi_{2} \xi_{3}+\left(\frac{1}{4} \nabla_{1} R_{2323}+\frac{1}{2} \nabla_{2} R_{1323}\right) \xi_{3}^{2}\right] \eta_{2}^{2} \\
& +\tau^{2}\left(\frac{1}{15} \xi_{1}^{2} \eta_{2}^{2}-\frac{2}{5} \xi_{1} \xi_{2} \eta_{1} \eta_{2}+\frac{2}{5} \xi_{2}^{2} \eta_{1}^{2}+\frac{1}{15} \xi_{2}^{2} \eta_{2}^{2}+\frac{2}{5} \xi_{3}^{2} \eta_{1}^{2}+\frac{2}{15} \xi_{3}^{2} \eta_{2}^{2}\right) \\
& +O\left(\varepsilon \tau^{2}+\tau^{4}\right)\left(\xi_{1} \xi_{2} \eta_{1} \eta_{2}+\xi_{1} \xi_{3} \eta_{1} \eta_{2}+\xi_{2}^{2} \eta_{1}^{2}+\xi_{2}^{2} \eta_{1} \eta_{2}+\xi_{3}^{2} \eta_{1}^{2}+\xi_{3}^{2} \eta_{1} \eta_{2}+\eta_{2}^{2}\right) \\
& =I_{1}+I I_{1}+I I I_{1}+I V_{1} \text {, }
\end{aligned}
$$

where $I_{1}$ (resp. $I I_{1}, I I I_{1}, I V_{1}$ ) collects all terms containing 0-power (resp. 1-power, 2-power and higher order powers) in $\tau$.

We estimate term by term from $I_{1}$ to $I V_{1}$. The key point is the fact that the combination of the zero order term and the second order term controls all others. 
The term $I_{1}$. - It is clear that $I_{1}=R_{m_{0}}(\xi, \eta, \xi, \eta)$. Recall the curvature approximation (1.3). Thus the term $I_{1}$ has the lower bound

$$
I_{1} \geqslant|\xi|^{2}|\eta|^{2}-\langle\xi, \eta\rangle^{2}=\left(\xi_{1} \eta_{2}-\xi_{2} \eta_{1}\right)^{2}+\xi_{3}^{2}\left(\eta_{1}^{2}+\eta_{2}^{2}\right) .
$$

The term $I I_{1}$. - The term $I I_{1}$ involves the first order parts $\tau \xi_{1}^{2} \eta_{2}^{2}, \tau \xi_{2}^{2} \eta_{1}^{2}$, $\tau \xi_{1} \xi_{2} \eta_{2}^{2}$ and $\tau \xi_{2}^{2} \eta_{1} \eta_{2}$. They cann't be directly controlled by the associated terms in the second order, but the combination of them composes good terms, more precisely, the term $I I_{1}$ can be stated as

$$
\begin{aligned}
I I_{1}= & \frac{3}{4} \tau \nabla_{1} R_{1212} \xi_{2}^{2} \eta_{1}^{2}-\tau \nabla_{1} R_{1212} \xi_{1} \xi_{2} \eta_{1} \eta_{2}+\frac{\tau}{4} \nabla_{1} R_{1212} \xi_{1}^{2} \eta_{2}^{2} \\
+ & \frac{\tau}{2} \nabla_{2} R_{1212}\left(\xi_{2}^{2} \eta_{1} \eta_{2}-\xi_{1} \xi_{2} \eta_{2}^{2}\right)+\frac{3}{2} \tau \nabla_{1} R_{1213} \xi_{2} \xi_{3} \eta_{1}^{2} \\
+ & \frac{3}{4} \tau \nabla_{1} R_{1313} \xi_{3}^{2} \eta_{1}^{2}+\tau\left[\left(\nabla_{1} R_{1223}+\nabla_{2} R_{1213}\right) \xi_{2} \xi_{3}\right. \\
& \left.\quad+\left(\nabla_{1} R_{1323}+\frac{1}{2} \nabla_{2} R_{1313}\right) \xi_{3}^{2}-\nabla_{1} R_{1213} \xi_{1} \xi_{3}\right] \eta_{1} \eta_{2} \\
+ & \tau\left[-\frac{1}{2}\left(\nabla_{1} R_{1223}+\nabla_{2} R_{1213}\right) \xi_{1} \xi_{3}+\frac{1}{2} \nabla_{2} R_{1223} \xi_{2} \xi_{3}\right. \\
& \left.\quad+\left(\frac{1}{4} \nabla_{1} R_{2323}+\frac{1}{2} \nabla_{2} R_{1323}\right) \xi_{3}^{2}\right] \eta_{2}^{2} \\
= & \frac{\tau}{4} \nabla_{1} R_{1212}\left(\xi_{2} \eta_{1}-\xi_{1} \eta_{2}\right)^{2}+\frac{\tau}{2} \nabla_{1} R_{1212}\left(\xi_{2} \eta_{1}-\xi_{1} \eta_{2}\right) \xi_{2} \eta_{1} \\
+ & \frac{\tau}{2} \nabla_{2} R_{1212}\left(\xi_{2} \eta_{1}-\xi_{1} \eta_{2}\right) \xi_{2} \eta_{2}+\frac{3}{2} \tau \nabla_{1} R_{1213} \xi_{2} \xi_{3} \eta_{1}^{2} \\
+ & \frac{3}{4} \tau \nabla_{1} R_{1313} \xi_{3}^{2} \eta_{1}^{2}+\tau\left[\left(\nabla_{1} R_{1223}+\nabla_{2} R_{1213}\right) \xi_{2} \xi_{3}\right. \\
& \left.+\left(\nabla_{1} R_{1323}+\frac{1}{2} \nabla_{2} R_{1313}\right) \xi_{3}^{2}-\nabla_{1} R_{1213} \xi_{1} \xi_{3}\right] \eta_{1} \eta_{2} \\
+ & \tau\left[-\frac{1}{2}\left(\nabla_{1} R_{1223}+\nabla_{2} R_{1213}\right) \xi_{1} \xi_{3}\right. \\
& \left.+\frac{1}{2} \nabla_{2} R_{1223} \xi_{2} \xi_{3}+\left(\frac{1}{4} \nabla_{1} R_{2323}+\frac{1}{2} \nabla_{2} R_{1323}\right) \xi_{3}^{2}\right] \eta_{2}^{2}
\end{aligned}
$$

Using the curvature approximation (1.6), Cauchy Schwartz inequality and the parallel property (2.1), we estimate the term $I I_{1}$ as follows

$$
\begin{aligned}
I I_{1} \geqslant-\varepsilon\left(\frac{\tau}{4}+\frac{1}{2}\right)( & \left.\xi_{1} \eta_{2}-\xi_{2} \eta_{1}\right)^{2}-\varepsilon \tau^{2} \xi_{1}^{2} \eta_{2}^{2}-2 \varepsilon \tau^{2} \xi_{2}^{2} \eta_{1}^{2} \\
& -\frac{1}{2} \varepsilon \tau^{2} \xi_{2}^{2} \eta_{2}^{2}-\varepsilon\left(\frac{5}{4}+\frac{3}{2} \tau\right) \xi_{3}^{2} \eta_{1}^{2}-\varepsilon\left(\frac{7}{4}+\frac{3}{2} \tau\right) \xi_{3}^{2} \eta_{2}^{2} .
\end{aligned}
$$


Here we drop the upper indicator for $\bar{\varepsilon}$ in (1.6). Now assume $0<\tau<2$, the following inequality holds

$$
\begin{aligned}
I I_{1} \geqslant-\varepsilon\left(\xi_{1} \eta_{2}-\xi_{2} \eta_{1}\right)^{2}-\varepsilon \tau^{2} \xi_{1}^{2} \eta_{2}^{2}-\frac{5}{4} \varepsilon \tau^{2} \xi_{2}^{2} \eta_{1}^{2} \\
\quad-\frac{1}{2} \varepsilon \tau^{2} \xi_{2}^{2} \eta_{2}^{2}-\varepsilon\left(\frac{5}{4}+\frac{3}{2} \tau\right) \xi_{3}^{2} \eta_{1}^{2}-\varepsilon\left(\frac{7}{4}+\frac{3}{2} \tau\right) \xi_{3}^{2} \eta_{2}^{2} .
\end{aligned}
$$

The term $I V_{1}$. - The remainder error term is negligible comparing with the second order of $\tau$ when $\varepsilon$ and $\tau$ are small. Let $\varepsilon_{1}$ be some small positive real number to be fixed later. Assume $0<\varepsilon<\frac{\varepsilon_{1}}{2}, 0<\tau<\frac{\varepsilon_{1}}{2}$ such that

$$
\begin{aligned}
I V_{1} & =O\left(\varepsilon \tau^{2}+\tau^{4}\right)\left(\xi_{1}^{2} \eta_{2}^{2}+\xi_{2}^{2} \eta_{1}^{2}+\xi_{2}^{2} \eta_{2}^{2}+\xi_{3}^{2} \eta_{1}^{2}+\xi_{3}^{2} \eta_{2}^{2}+\eta_{2}^{2}\right) \\
& \geqslant-\varepsilon_{1} \tau^{2}\left(\xi_{2}^{2} \eta_{1}^{2}+\xi_{3}^{2} \eta_{1}^{2}+\eta_{2}^{2}\right) .
\end{aligned}
$$

Here we use the fact $\xi_{1}^{2}+\xi_{2}^{2}+\xi_{3}^{2}=1$. Gathering (3.6), (3.7), (3.8) and (3.9), we estimate

$$
\begin{aligned}
\mathcal{C} & \left.m_{0}, \nu_{0}\right)(\xi, \eta) \\
\geqslant & \left(\xi_{1} \eta_{2}-\xi_{2} \eta_{1}\right)^{2}+\xi_{3}^{2}\left(\eta_{1}^{2}+\eta_{2}^{2}\right)-\frac{1}{2} \varepsilon_{1}\left(\xi_{1} \eta_{2}-\xi_{2} \eta_{1}\right)^{2}-\frac{1}{2} \varepsilon_{1} \tau^{2} \xi_{1}^{2} \eta_{2}^{2} \\
& -\frac{5}{8} \varepsilon_{1} \tau^{2} \xi_{2}^{2} \eta_{1}^{2}-\frac{1}{4} \varepsilon_{1} \tau^{2} \xi_{2}^{2} \eta_{2}^{2}-\varepsilon_{1}\left(\frac{5}{8}+\frac{3}{4} \tau\right) \xi_{3}^{2} \eta_{1}^{2}-\varepsilon_{1}\left(\frac{7}{8}+\frac{3}{4} \tau\right) \xi_{3}^{2} \eta_{2}^{2} \\
& +\tau^{2}\left(\frac{1}{15} \xi_{1}^{2} \eta_{2}^{2}-\frac{2}{5} \xi_{1} \xi_{2} \eta_{1} \eta_{2}+\frac{2}{5} \xi_{2}^{2} \eta_{1}^{2}+\frac{1}{15} \xi_{2}^{2} \eta_{2}^{2}+\frac{2}{5} \xi_{3}^{2} \eta_{1}^{2}+\frac{2}{15} \xi_{3}^{2} \eta_{2}^{2}\right) \\
& -\varepsilon_{1} \tau^{2}\left(\xi_{2}^{2} \eta_{1}^{2}+\xi_{3}^{2} \eta_{1}^{2}+\eta_{2}^{2}\right) \\
= & \left(1-\frac{\varepsilon_{1}}{2}\right)\left(\xi_{1} \eta_{2}-\xi_{2} \eta_{1}\right)^{2}+\left(\frac{1}{15}-\frac{\varepsilon_{1}}{2}\right) \tau^{2} \xi_{1}^{2} \eta_{2}^{2}-\frac{2}{5} \tau^{2} \xi_{1} \xi_{2} \eta_{1} \eta_{2} \\
& +\left(\frac{2}{5}-\frac{5}{8} \varepsilon_{1}\right) \tau^{2} \xi_{2}^{2} \eta_{1}^{2}+\left(\frac{1}{15}-\frac{\varepsilon_{1}}{4}\right) \tau^{2} \xi_{2}^{2} \eta_{2}^{2}+\left[\frac{2}{5} \tau^{2}-\varepsilon_{1}\left(\frac{5}{8}+\frac{3}{4} \tau\right)+1\right] \xi_{3}^{2} \eta_{1}^{2} \\
& +\left[\frac{2}{15} \tau^{2}-\varepsilon_{1}\left(\frac{7}{8}+\frac{3}{4} \tau\right)+1\right] \xi_{3}^{2} \eta_{2}^{2}-\varepsilon_{1} \tau^{2}\left(\xi_{2}^{2} \eta_{1}^{2}+\xi_{3}^{2} \eta_{1}^{2}+\eta_{2}^{2}\right) \\
= & {\left[1-\varepsilon_{1}+\left(\frac{1}{15}-2 \varepsilon_{1}\right) \tau^{2}\right] \xi_{1}^{2} \eta_{2}^{2}-2\left(1-\varepsilon_{1}+\frac{\tau^{2}}{5}\right) \xi_{1} \xi_{2} \eta_{1} \eta_{2} } \\
& +\left[1-\varepsilon_{1}+\left(\frac{2}{5}-2 \varepsilon_{1}\right) \tau^{2}\right] \xi_{2}^{2} \eta_{1}^{2}+\frac{\varepsilon_{1}}{2}\left(\xi_{1} \eta_{2}-\xi_{2} \eta_{1}\right)^{2} \\
& +\left[1-\varepsilon_{1}\left(\frac{5}{8}+\frac{3}{4} \tau+\tau^{2}\right)\right] \xi_{3}^{2} \eta_{1}^{2}+\left[1-\varepsilon_{1}\left(\frac{7}{8}+\frac{3}{4} \tau\right)\right] \xi_{3}^{2} \eta_{2}^{2}+\frac{3}{2} \varepsilon_{1} \tau^{2} \xi_{1}^{2} \eta_{2}^{2} \\
& +\frac{3}{8} \varepsilon_{1} \tau^{2} \xi_{2}^{2} \eta_{1}^{2}+\left(\frac{1}{15}-\frac{\varepsilon_{1}}{4}\right) \tau^{2} \xi_{2}^{2} \eta_{2}^{2}+\frac{2}{5} \tau^{2} \xi_{3}^{2} \eta_{1}^{2}+\frac{2}{15} \tau^{2} \xi_{3}^{2} \eta_{2}^{2}-\varepsilon_{1} \tau^{2} \eta_{2}^{2}
\end{aligned}
$$


The discriminant of the quadratic polynomial

$h_{\varepsilon, \tau}(t)=\left[1-\varepsilon+\left(\frac{1}{15}-2 \varepsilon\right) \tau^{2}\right] t^{2}-2\left(1-\varepsilon+\frac{\tau^{2}}{5}\right) t+1-\varepsilon+\left(\frac{2}{5}-2 \varepsilon\right) \tau^{2}$

is polynomial with arguments $(\varepsilon, \tau)$. By continuity, there exists a small positive constant $\widetilde{\varepsilon}_{1}$ such that the discriminant is non-positive in $\left[0, \widetilde{\varepsilon}_{1}\right] \times\left[0, \widetilde{\varepsilon}_{1}\right]$. Together with the fact $\xi_{1}^{2}+\xi_{2}^{2}+\xi_{2}^{3}=1$, we have

$$
\begin{aligned}
\mathcal{C}\left(m_{0}, \nu_{0}\right)(\xi, \eta) \geqslant & \frac{\varepsilon_{1}}{2}\left(\xi_{1} \eta_{2}-\xi_{2} \eta_{1}\right)^{2}+\left[1-\varepsilon_{1}\left(\frac{5}{8}+\frac{3}{4} \tau+\tau^{2}\right)\right] \xi_{3}^{2} \eta_{1}^{2} \\
& +\left[1-\varepsilon_{1}\left(\frac{7}{8}+\frac{3}{4} \tau\right)\right] \xi_{3}^{2} \eta_{2}^{2}+\frac{1}{2} \varepsilon_{1} \tau^{2} \xi_{1}^{2} \eta_{2}^{2}+\frac{3}{8} \varepsilon_{1} \tau^{2} \xi_{2}^{2} \eta_{1}^{2} \\
& +\left(\frac{1}{15}-\frac{5 \varepsilon_{1}}{4}\right) \tau^{2} \xi_{2}^{2} \eta_{2}^{2}+\frac{2}{5} \tau^{2} \xi_{3}^{2} \eta_{1}^{2}+\left(\frac{2}{15}-\varepsilon_{1}\right) \tau^{2} \xi_{3}^{2} \eta_{2}^{2} .
\end{aligned}
$$

Thus, we could choose some small $\varepsilon_{1}<\widetilde{\varepsilon}_{1}$ such that for any $\tau<\frac{\varepsilon_{1}}{2}$ there holds $1-\varepsilon_{1}\left(\frac{5}{8}+\frac{3}{4} \tau+\tau^{2}\right)>0$ and $1-\varepsilon_{1}\left(\frac{7}{8}+\frac{3}{4} \tau\right)>0$. As a consequence, there exist the constants $\widetilde{\varepsilon}_{0}, \kappa_{1}$ and $\delta_{1}$ such that $\forall \varepsilon<\widetilde{\varepsilon}_{0}$ and $\forall \nu_{0} \in T_{m_{0}} M$ with $\left|\nu_{0}\right|<\delta_{1}$, we have

$$
\mathcal{C}\left(m_{0}, \nu_{0}\right)(\xi, \eta) \geqslant \kappa_{1}\left[\left(|\xi|^{2}|\eta|^{2}-\langle\xi, \eta\rangle^{2}\right)+\left|\nu_{0}\right|^{2}\left(\xi_{2}^{2}+\xi_{3}^{2}\right)|\eta|^{2}+\left|\nu_{0}\right|^{2}|\xi|^{2} \eta_{2}^{2}\right] .
$$

Finally, we prove Theorem 3.1.

\subsubsection{Behaviour near the focalization}

Under the curvature assumption (1.3) and (1.4) (or (1.5)) with $\varepsilon$ small enough, we can obtain by the method in [17] near the focalization.

THEOREM 3.2. - Under the same assumptions as in Theorem 1.1, there exist some universal positive constants $\widetilde{\varepsilon}_{0,1}, \delta_{2} \in\left(\frac{3 \pi}{4}, \pi\right), \kappa_{1,1}$ such that if $\varepsilon<\widetilde{\varepsilon}_{0,1}$, then for all $\left(m_{0}, \nu_{0}\right) \in$ NoCut with $\delta_{2}<\tau=\left|\nu_{0}\right|<t_{F}\left(m_{0}, \nu_{0}\right)$ and for all tangent vectors $\xi, \eta$ in $T_{m_{0}} M$

$$
\mathcal{C}\left(m_{0}, \nu_{0}\right)(\xi, \eta) \geqslant \kappa_{1,1}\left(|\xi \wedge \eta|_{m_{0}}^{2}+|\xi|_{m_{0}}^{2}\left|\eta \wedge \nu_{0}\right|_{m_{0}}^{2}+\left|\xi \wedge \nu_{0}\right|_{m_{0}}^{2}|\eta|_{m_{0}}^{2}\right) \text {. }
$$

Proof. - In view of [17, (5.22)], there exist positive numbers $\kappa_{2}, \varepsilon_{2}, \delta_{2} \in$ $\left(\frac{3 \pi}{4}, \pi\right)$ such that if $\varepsilon<\varepsilon_{2}$, then for all $\left(m_{0}, \nu_{0}\right) \in$ NoCut with $\delta_{2}<\tau=$ $\left|\nu_{0}\right|<t_{F}\left(m_{0}, \nu_{0}\right)$ and for all tangent vectors $\xi, \eta$ in $T_{m_{0}} M$ with $|\xi|=|\eta|=1$, the $\mathcal{C}$-curvature has the following estimate

$$
\mathcal{C}\left(m_{0}, \nu_{0}\right)(\xi, \eta) \geqslant \kappa_{2}\left(\left|S^{\perp} \xi\right|^{2}|\eta|^{2}+\xi_{1}^{2} \eta_{2}^{2}\right),
$$

where $S^{\perp}$ denotes the orthogonal projection of $S$ on the orthogonal subspace $\nu_{0}^{\perp}$. 
From the curvature assumption (1.3), the Hessian comparison theorem [4] infers

$$
-S^{\perp} \geqslant-\frac{\left|\nu_{0}\right| \cos \left|\nu_{0}\right|}{\sin \left|\nu_{0}\right|} I_{n-1} .
$$

Hence the term $\left|S^{\perp} \xi\right|^{2}$ controls $\xi_{2}^{2}+\xi_{3}^{2}$ if $\left|\nu_{0}\right| \geqslant \frac{3 \pi}{4}$, i.e. $\left|S^{\perp} \xi\right|^{2} \geqslant 2\left(\xi_{2}^{2}+\xi_{3}^{2}\right)$. By Cauchy-Schwarz inequality, we estimate

$$
\frac{1}{2}\left(\xi_{1}^{2} \eta_{2}^{2}+\xi_{2}^{2} \eta_{1}^{2}\right) \geqslant \frac{1}{4}\left(\xi_{1} \eta_{2}-\xi_{2} \eta_{1}\right)^{2}
$$

Thus, it follows from (3.11),

$$
\begin{aligned}
\mathcal{C}\left(m_{0}, \nu_{0}\right)(\xi, \eta) & \geqslant \kappa_{2}\left[\frac{1}{2} \xi_{1}^{2} \eta_{2}^{2}+\frac{1}{4}\left(\xi_{1} \eta_{2}-\xi_{2} \eta_{1}\right)^{2}+\left(\xi_{2}^{2}+\xi_{3}^{2}\right)|\eta|^{2}\right] \\
& \geqslant \kappa_{2}\left[\frac{1}{2} \xi_{1}^{2} \eta_{2}^{2}+\frac{1}{4} \xi_{2}^{2} \eta_{1}^{2}-\frac{1}{2} \xi_{1} \xi_{2} \eta_{1} \eta_{2}+\left(\xi_{2}^{2}+\xi_{3}^{2}\right)|\eta|^{2}\right] \\
& =\kappa_{2}\left[\frac{1}{4}\left(|\xi|^{2}|\eta|^{2}-\langle\xi, \eta\rangle^{2}\right)+\frac{1}{4} \xi_{1}^{2} \eta_{2}^{2}+\xi_{2}^{2}|\eta|^{2}+\frac{3}{4} \xi_{3}^{2}|\eta|^{2}\right] \\
& \geqslant \kappa_{2}\left[\left(|\xi|^{2}|\eta|^{2}-\langle\xi, \eta\rangle^{2}\right)+\left(\xi_{2}^{2}+\xi_{3}^{2}\right)|\eta|^{2}+|\xi|^{2} \eta_{2}^{2}\right] \\
& \geqslant C \kappa_{2}\left[\left(|\xi|^{2}|\eta|^{2}-\langle\xi, \eta\rangle^{2}\right)+\left|\nu_{0}\right|^{2}\left(\xi_{2}^{2}+\xi_{3}^{2}\right)|\eta|^{2}+\left|\nu_{0}\right|^{2}|\xi|^{2} \eta_{2}^{2}\right] .
\end{aligned}
$$

Therefore, there exist positive constants $\widetilde{\varepsilon}_{0,1}<\varepsilon_{2}, \kappa_{1,1}<C \kappa_{2}$ such that (3.10) holds. Finally, the proof of Theorem 3.2 is complete.

\subsubsection{Behaviour in the intermediate case}

In this subsection we assume $\delta_{1} \leqslant\left|\nu_{0}\right| \leqslant \delta_{2}<t_{F}\left(m_{0}, \nu_{0}\right)$, where the positive constant $\delta_{2}$ is strictly smaller than $\pi$ and given in Theorem 3.2, and the positive constant $\delta_{1}$ is given in Theorem 3.1. We adapt the same strategy in $[8$, Theorem 2] to get the stability result. For convenience of readers, we recast as follows.

Proposition 3.3. - Let $(M, g)$ be a closed n-dimensional Riemannian manifold satisfying (1.3) and (1.4) (or (1.5)) with $\varepsilon<\gamma$. Let $\left(m_{0}, \nu_{0}\right) \in$ NoCut with $\tau=\left|\nu_{0}\right| \leqslant \delta_{2}$. Assume $\gamma$ is small enough such that $\delta_{2}<$ $t_{F}\left(m_{0}, \nu_{0}\right)$ and

$$
\frac{\left|\nu_{0}\right|}{\sin \left|\nu_{0}\right|} \gamma \leqslant \frac{1}{4 \sqrt{n-1}}
$$

Then there exists a positive constant $C_{1} \geqslant 1$ under control (independent of $\left.\left(m_{0}, \nu_{0}, \gamma\right)\right)$ such that for all $\xi, \eta \in T_{m_{0}} M$ the following inequality holds

$$
\left|\mathcal{C}\left(m_{0}, \nu_{0}\right)(\xi, \eta)-\overline{\mathcal{C}}\left(m_{0}, \nu_{0}\right)(\xi, \eta)\right| \leqslant C_{1}\left(\frac{\left|\nu_{0}\right|}{\sin \left|\nu_{0}\right|}\right)^{4} \gamma\left(\left|\xi^{\perp}\right|^{2}+\left|\eta^{\perp}\right|^{2}\right),
$$


where $\xi^{\perp}$ (resp. $\left.\eta^{\perp}\right)$ is orthogonal projection of $\xi$ (resp. $\left.\eta\right)$ on the orthogonal subspace $\nu_{0}^{\perp}$.

Proof. - The $\mathcal{C}$-curvature difference has the following expression

$$
\begin{aligned}
\mathcal{C}\left(m_{0}, \nu_{0}\right)(\xi, \eta)-\overline{\mathcal{C}}\left(m_{0}, \nu_{0}\right)(\xi, \eta) & \\
= & -\frac{3}{2}\langle(\ddot{S}-\ddot{\bar{S}}) \xi, \xi\rangle \eta_{1}^{2} \\
+ & 3\left[-\frac{1}{\tau}\left\langle\dot{S}^{\prime} \xi, \xi\right\rangle+\frac{1}{\tau^{2}}\left\langle S^{\prime} \xi, \xi\right\rangle-\frac{2}{\tau}\left\langle(\dot{S}-\dot{\bar{S}}) \xi, P \xi^{\perp}\right\rangle+\frac{2}{\tau^{2}}\left\langle(S-\bar{S}) \xi, P \xi^{\perp}\right\rangle\right] \eta_{1} \eta_{2} \\
+ & \frac{3}{2}\left[-\frac{1}{\tau^{2}}\left\langle S^{\prime \prime} \xi, \xi\right\rangle-\frac{1}{\tau}\langle(\dot{S}-\dot{\bar{S}}) \xi, \xi\rangle-\frac{4}{\tau^{2}}\left\langle S^{\prime} P \xi^{\perp}, \xi\right\rangle\right. \\
& \left.\quad-\frac{2}{\tau^{2}}\left\langle(S-\bar{S}) P \xi^{\perp}, P \xi^{\perp}\right\rangle+\frac{2}{\tau^{2}}\langle(S-\bar{S}) \xi, P \xi\rangle\right] \eta_{2}^{2} .
\end{aligned}
$$

Plugging (B.28) and (B.29) into (3.12) we get the result. Therefore, Proposition 3.3 is proved.

As a consequnce, we get the stability result as follows.

ThEOREM 3.4. - Under the same assumptions as in Theorem 1.1, there exist some universal positive constants $\widetilde{\varepsilon}_{0,2}, \kappa_{1,2}$ such that if $\varepsilon<\widetilde{\varepsilon}_{0,2}$, then for all $\left(m_{0}, \nu_{0}\right) \in$ NoCut with $\delta_{1} \leqslant \tau=\left|\nu_{0}\right| \leqslant \delta_{2}<t_{F}\left(m_{0}, \nu_{0}\right)$ and for all tangent vectors $\xi, \eta$ in $T_{m_{0}} M$

$$
\mathcal{C}\left(m_{0}, \nu_{0}\right)(\xi, \eta) \geqslant \kappa_{1,2}\left(|\xi \wedge \eta|_{m_{0}}^{2}+|\xi|_{m_{0}}^{2}\left|\eta \wedge \nu_{0}\right|_{m_{0}}^{2}+\left|\xi \wedge \nu_{0}\right|_{m_{0}}^{2}|\eta|_{m_{0}}^{2}\right) \text {. }
$$

Proof. - Assume $0<\varepsilon<\frac{\sin \delta_{2}}{4 \delta_{2} \sqrt{n-1}}$. We note the function $\frac{t}{\sin t}$ is nondecreasing in the interval $[0, \pi)$, and the condition in Proposition 3.3 is satisfied. Thus, we have

$$
\begin{aligned}
\mathcal{C}\left(m_{0}, \nu_{0}\right)(\xi, \eta) & \geqslant \overline{\mathcal{C}}\left(m_{0}, \nu_{0}\right)(\xi, \eta)-C_{1}\left(\frac{\delta_{2}}{\sin \delta_{2}}\right)^{4} \varepsilon\left(\xi_{2}^{2}+\xi_{3}^{2}+\eta_{2}^{2}\right) \\
& \geqslant \bar{\kappa}_{2}\left(|\xi|^{2}|\eta|^{2}-\langle\xi, \eta\rangle^{2}\right)+\left(\widetilde{\kappa}_{0} \tau^{2}-\frac{C_{1} \delta_{2}^{4}}{\sin ^{4} \delta_{2}} \varepsilon\right)\left(\xi_{2}^{2}+\xi_{3}^{2}+\eta_{2}^{2}\right) \\
& \geqslant \bar{\kappa}_{2}\left(|\xi|^{2}|\eta|^{2}-\langle\xi, \eta\rangle^{2}\right)+\left(\widetilde{\kappa}_{0}-\frac{C_{1} \delta_{2}^{4}}{\delta_{1}^{2} \sin ^{4} \delta_{2}} \varepsilon\right) \tau^{2}\left(\xi_{2}^{2}+\xi_{3}^{2}+\eta_{2}^{2}\right) \\
& \geqslant \frac{\bar{\kappa}_{2}}{2}\left[|\xi|^{2}|\eta|^{2}-\langle\xi, \eta\rangle^{2}+\tau^{2}\left(\xi_{2}^{2}+\xi_{3}^{2}+\eta_{2}^{2}\right)\right] \\
& \geqslant \kappa_{1,2}\left[\left(|\xi|^{2}|\eta|^{2}-\langle\xi, \eta\rangle^{2}\right)+\left|\nu_{0}\right|^{2}\left(\xi_{2}^{2}+\xi_{3}^{2}\right)|\eta|^{2}+\left|\nu_{0}\right|^{2}|\xi|^{2} \eta_{2}^{2}\right] .
\end{aligned}
$$

Here we choose $\varepsilon<\widetilde{\varepsilon}_{1,2}:=\min \left(\frac{\bar{\kappa}_{2} \delta_{1}^{3} \sin ^{4} \delta_{2}}{2 C_{1} \delta_{2}^{4}}, \frac{\sin \delta_{2}}{4 \delta_{2} \sqrt{n-1}}\right)$ and $\kappa_{1,2}<\frac{\bar{\kappa}_{2}}{2}$. Finally, we prove the result.

Proof of Theorem 1.1. - It is a direct consequence of Theorems 3.1, 3.2 and 3.4. 
Proof of Corollary 1.2. - Thanks to Theorem 1.1, NNCC conidtion holds. Such condition is stable for Riemannian product. On the other hand, NNCC condition implies A3W condition. Thus, the desired result yields.

\section{Proof of Theorem 1.3}

In this section, we are going to prove Theorem 1.3. Let ( $\left.\rho_{0} \mathrm{dvol}, \rho_{1} \mathrm{dvol}\right)$ be $C^{k, \alpha}$ positive Borel probability measures on $M$. The curvatures of $M$ satisfy (1.3) and (1.4) (or (1.5)). Fix any couple $(k, \alpha) \in \mathbb{N} \times(0,1)$, with $k \geqslant 2$. Let $\operatorname{Hess}^{(c)} u$ be c-Hessian of $u$, namely,

$$
\operatorname{Hess}^{(c)} u=\nabla_{m}^{2} u+\nabla_{m}^{2} c\left(\cdot, \exp _{m} \nabla_{m} u\right) .
$$

On the one hand, a $C^{2}$ potential function $u$ of the optimal transport map $G(m)=\exp _{m} \nabla_{m} u$ pushing forward $\rho_{0}$ dvol to $\rho_{1}$ dvol satisfies the following Monge-Ampère equation

$$
\operatorname{det}\left(d_{\nabla_{m} u} \exp _{m}\right) \operatorname{det} \operatorname{Hess}^{(c)} u=\frac{\rho_{0}(m)}{\rho_{1}\left(\exp _{m} \nabla_{m} u\right)} .
$$

On the other hand, a classical $C^{2}$ solution of the above equation is the potential function of the optimal transport map $\exp _{m} \nabla_{m} u$ pushes forward $\rho_{0}$ dvol to $\rho_{1}$ dvol. To establish Theorem 1.3, it suffices to prove the Monge-Ampère equation admits a classical $C^{k+2, \alpha}$ solution. We attack it by the continuity method. Here we consider only regularity issue on simply connected manifold $M$. For the general case, we reduce to simply connected manifold by covering arguments (see [8]).

Let $\mathcal{I}$ be the set of the parameter $t \in[0,1]$ for which there exists a $C^{k+2, \alpha}$ solution $u_{t}$ of the equation (4.1) with $\rho_{1}$ replaced by $\rho_{t}=(1-t) \rho_{0}+t \rho_{1}$. It is clear that $0 \in \mathcal{I}$, so the set $\mathcal{I}$ is not empty. The openness is derived by the implicit function theorem [19]. If $\mathcal{I}$ is closed, the connectedness of the set $[0,1]$ shows $\mathcal{I}=[0,1]$ and we prove the equation (4.1) admits a $C^{k, \alpha}$ solution. From Theorem 1.1, we know that on the Riemannian manifold with the curvature conditions (1.3) and (1.4) (or (1.5)), A3S condition holds. [7, Theorem 6.1] (see also [8]) reduced the closedness of the set $\mathcal{I}$ to the following estimate

MAIN ESTIMATE. - There exists a positive number $\delta_{0}$ (depending on the densities) under control such that

$$
\operatorname{det} d_{\nabla_{m} u_{t}} \exp _{m} \geqslant \delta_{0}
$$

for each $(t, m) \in \mathcal{I} \times M$. 
Fix $t \in \mathcal{I}$. From [7, Theorem 3.1], we know that $\nabla u_{t}$ at $m$ locates in the injectivity domain at $m$. Recalling the curvature assumption (1.3), by Rauch comparison theorem [2], the length $\left|\nabla_{m} u_{t}\right|$ is strictly less than $\pi$. The Jacobian is related to Jacobi field, i.e.

$$
\operatorname{det} d_{\nabla_{m} u_{t}} \exp _{m}=\operatorname{det} J_{0}\left(m, \nabla_{m} u_{t}, 1\right)
$$

By Bishop's theorem[4], $\operatorname{det} d_{\nabla_{m} u_{t}} \exp _{m}$ is uniformly bounded above by 1 if $M$ has nonnegative Ricci curvature. It is known that $\operatorname{det} d_{\nabla_{m} u_{t}} \exp _{m}$ is positive. But det $d_{\nabla_{m} u_{t}} \exp _{m}$ may not has a positive lower bound. Since det $J_{0}(m, \nu, 1)$ vanishes if (and only if) $\exp _{m} \nu$ is conjugate to $m$, so the estimate (4.2) is not obvious. For instance, on the sphere $\mathbb{S}^{n}$ with the standard metric, det $d_{\nabla_{m} u_{t}} \exp _{m}=\left(\frac{\sin \left|\nabla_{m} u_{t}\right|}{\left|\nabla_{m} u_{t}\right|}\right)^{n-1}$ is close to zero when $\left|\nabla_{m} u_{t}\right|$ is close to $\pi$. Making use of Lemma A.2 in Appendix, the estimate (4.2) is obvious if $\max \left\{\left|\nabla_{m} u_{t}\right|: m \in M\right\} \leqslant \frac{3 \pi}{4}$, provided $\varepsilon$ is small enough. Thus without loss of generality, we assume there exists at least a point such that the length of gradient $\left|\nabla u_{t}\right|$ at that point is not smaller than $\frac{3 \pi}{4}$. To prove (4.2) we need to construct an appropriate test function.

Let $\mathcal{J}\left(m, \nabla_{m} u_{t}\right)=-\left|\nabla_{m} u_{t}\right|^{2} S^{-1}\left(m, \nabla_{m} u_{t}, 1\right)$. We consider the minimization problem

$$
\min \left\{\langle\mathcal{J} \xi, \xi\rangle:(m, \xi) \in T M, \frac{3 \pi}{4} \leqslant\left|\nabla u_{t}\right|_{m},|\xi|_{m}=1, \xi \perp \nabla_{m} u_{t}\right\} .
$$

Let $\left(m_{0}, \xi_{0}\right)$ be the minimum point. We consider the test function

$$
h(m, \xi)=\frac{\langle\mathcal{J} \xi, \xi\rangle+\left\langle\xi, \nabla u_{t}\right\rangle^{2}}{|\xi|^{2}-\frac{\left\langle\xi, \nabla u_{t}\right\rangle^{2}}{\left|\nabla u_{t}\right|^{2}}} .
$$

Then $h$ attains the minimum at the point $\left(m_{0}, \xi_{0}\right)$ in a neighborhood of the point $\left(m_{0}, \xi_{0}\right)$ in $T M$. To see this, let $\xi^{\perp}=\xi-\left\langle\xi, \nabla u_{t}\right\rangle \frac{\nabla u_{t}}{\left|\nabla u_{t}\right|^{2}}$ be the orthogonal projection of $\xi$ on $\left(\nabla_{m} u\right)^{\perp}$. Then

$$
\frac{\left\langle\mathcal{J} \xi^{\perp}, \xi^{\perp}\right\rangle}{\left|\xi^{\perp}\right|^{2}}=h(m, \xi) \text {. }
$$

By continuity, we obtain $h$ attains local minimum at the point $\left(m_{0}, \xi_{0}\right)$. The minimum $h\left(m_{0}, \xi_{0}\right)$ has a nice explanation: the second eigenvalue of $\mathcal{J}$. Specifically, as $h$ is bilinear with respect to $\xi$ on the orthogonal subspace $\left(\nabla_{m} u\right)^{\perp}$, the minimum $h\left(m_{0}, \xi_{0}\right)$ is the second eigenvalue of the self adjoint operator $\mathcal{J}\left(m_{0}, \nabla_{m_{0}} u\right)$ with the associated eigenvector $\xi_{0}$. As a consequence of the above explanation, a necessary condition for the main estimate (4.2) is that the minimum $h\left(m_{0}, \xi_{0}\right)$ has a positive lower bound. At first the minimum $h\left(m_{0}, \xi_{0}\right)$ has to be positive. To see this, from the Hessian Comparison Theorem, we know that $-S^{\perp}$ is positive definite. Thus the minimum $h\left(m_{0}, \xi_{0}\right)$ is positive and has a lower bound under control. To differentiate 
the test function $h$, it needs to rule out the boundary case. Proposition B.8 infers

$$
h\left(m_{0}, \xi_{0}\right) \geqslant-\frac{\left|\nabla_{m_{0}} u_{t}\right| \sin \left|\nabla_{m_{0}} u_{t}\right|}{\cos \left|\nabla_{m_{0}} u_{t}\right|}-C_{8} \varepsilon,
$$

provided $\varepsilon<\gamma_{1}$. Since the function $-\frac{r \sin r}{\cos r}$ is non-increasing in $\left(\frac{\pi}{2}, \pi\right)$, we can assume $\left|\nabla_{m_{0}} u_{t}\right|>\pi-\delta, 0<\delta<\frac{\pi}{4}$. Henceforth, we drop the subscript $t$ and set for short $\tau=\left|\nabla_{m_{0}} u\right|$.

Some local notations. - We take the Fermi coordinate system $x=$ $\left(x^{1}, x^{2}, \ldots, x^{n}\right)$ along the geodesic $\exp _{m_{0}}\left(s \nabla_{m_{0}} u\right)$ as the one constructed in section 2 and $v=\left(v^{1}, v^{2}, \ldots, v^{n}\right)$ be the fiber coordinates of $T M \rightarrow M$ naturally associated to $x$. We abbreviate the partial derivatives as follows

$$
\partial_{i}=\frac{\partial}{\partial x^{i}}, \quad \partial_{i j}^{2}=\frac{\partial^{2}}{\partial x^{i} \partial x^{j}}, \quad D_{i}=\frac{\partial}{\partial v^{i}}, \quad D_{i j}^{2}=\frac{\partial^{2}}{\partial v^{i} \partial v^{j}} .
$$

Components of tensors will be denoted by

$$
\begin{aligned}
\operatorname{grad} u & =\nabla^{i} u(m) \frac{\partial}{\partial x^{i}}, & \nabla_{m}^{2} u & =\nabla_{j}^{i} u(m) \mathrm{d} x^{j} \otimes \frac{\partial}{\partial x^{i}}, \\
S & =S_{j}^{i}(m, \nu, 1) \mathrm{d} x^{j} \otimes \frac{\partial}{\partial x^{i}}, & \mathcal{J} & =\mathcal{J}_{j}^{i}(m, \nu) \mathrm{d} x^{j} \otimes \frac{\partial}{\partial x^{i}}, \\
\mathcal{H} & =\mathcal{H}_{j}^{i}(m, \nu) \mathrm{d} x^{j} \otimes \frac{\partial}{\partial x^{i}}, & \mathcal{F} & =\mathcal{F}_{j}^{i}(m, \nu) \mathrm{d} x^{j} \otimes \frac{\partial}{\partial x^{i}},
\end{aligned}
$$

where $\mathcal{H}_{j}^{i}=\nabla_{j}^{i} u+S_{j}^{i}, \mathcal{H}_{k}^{i} \mathcal{F}_{j}^{k}=\delta_{j}^{i}$.

We denote $D^{k}=g^{k l} D_{l}$ and $\partial^{k}=g^{k l} \partial_{l}$ the lifting indices. Let us now describe the behavior of the components $\mathcal{J}_{\beta}^{\alpha}$.

Proposition 4.1. - Let $\left(m_{0}, \nu_{0}\right) \in$ NoCut and $\left|\nu_{0}\right| \geqslant \pi-\delta$ with $0<$ $\delta<\frac{\pi}{4}$. Let $x$ be the Fermi coordinate system associated to the geodesic $\exp _{m_{0}} s \nu_{0}$ and $v$ be the fiber coordinates of $T M \rightarrow M$ naturally associated to $x$. Then there exists a positive constant $C$ under control such that the absolute value of the first and second partial derivatives of the components $\mathcal{J}_{\beta}^{\alpha}, \alpha, \beta \in$ $\{2, \ldots, n\}$ with respect to $(x, v)$, evaluated at the point $\left(0,\left(\left|\nu_{0}\right|, 0\right), 1\right)$, are all bounded above by $C \varepsilon$, except the following partial derivatives

$$
D_{1} \mathcal{J}_{\alpha}^{\alpha}, \quad \partial_{\beta \beta} \mathcal{J}_{\alpha}^{\alpha}, \quad D_{11} \mathcal{J}_{\alpha}^{\alpha}, \quad D_{\beta \beta} \mathcal{J}_{\alpha}^{\alpha}, \quad D_{\varphi \psi} \mathcal{J}_{\iota}^{\phi}, \quad \psi \neq \varphi, \iota \neq \phi .
$$

and the following estimates hold

$$
\begin{aligned}
\left|D_{1} \mathcal{J}_{\alpha}^{\alpha}+\pi\right| & \leqslant C(\varepsilon+\delta), \\
\left|\partial_{\beta \beta} \mathcal{J}_{\alpha}^{\alpha}-\pi^{2}\right| & \leqslant C(\varepsilon+\delta), \\
\left|D_{11} \mathcal{J}_{\alpha}^{\alpha}+2\right| & \leqslant C(\varepsilon+\delta), \\
\left|D_{\beta \beta} \mathcal{J}_{\alpha}^{\alpha}+1+2 \delta_{\alpha \beta}\right| & \leqslant C(\varepsilon+\delta), \\
\left|D_{\varphi \psi} \mathcal{J}_{\iota}^{\phi}+\delta_{\phi \varphi} \delta_{\iota \psi}+\delta_{\phi \psi} \delta_{\iota \varphi}\right| & \leqslant C(\varepsilon+\delta), \quad \psi \neq \varphi, \iota \neq \phi .
\end{aligned}
$$


Proof. - Recalling Proposition B.8, we only need to calculate the following components

$$
\partial_{x} \overline{\mathcal{J}}_{\beta}^{\alpha}, \quad D_{v} \overline{\mathcal{J}}_{\beta}^{\alpha}, \quad \partial_{x x} \overline{\mathcal{J}}_{\beta}^{\alpha}, \quad D_{x v} \overline{\mathcal{J}}_{\beta}^{\alpha}, \quad D_{v v} \overline{\mathcal{J}}_{\beta}^{\alpha}
$$

where $\overline{\mathcal{J}}=-|\nu|^{2} \bar{S}^{-1}(m, \nu, 1)$. By differentiating the equation

$$
\langle\overline{\mathcal{J}} \xi, \xi\rangle=f(|\nu|)|\xi|^{2}-\left(|\nu|^{2}+f(|\nu|)\right)\left\langle\xi, \frac{\nu}{|\nu|}\right\rangle^{2} \text {, where } f(|\nu|)=-\frac{|\nu| \sin |\nu|}{|\nu|} .
$$

Evaluated at the point $\left(m_{0}, \nu_{0}, \xi\right)$ with $\xi \perp \nu_{0}$ and $|\xi|=1$, we get

$$
\begin{aligned}
\partial_{i} \overline{\mathcal{J}}_{\beta}^{\alpha} \xi_{\alpha} \xi^{\beta}= & 0, \quad D_{i} \overline{\mathcal{J}}_{\beta}^{\alpha} \xi^{\alpha} \xi^{\beta}=\dot{f} \delta_{1 i}, \\
\partial_{i j} \overline{\mathcal{J}}_{\beta}^{\alpha} \xi_{\alpha} \xi^{\beta}= & -\left|\nu_{0}\right| \dot{f}\left(\left|\nu_{0}\right|\right), \quad \partial_{i} D_{j} \overline{\mathcal{J}}_{\beta}^{\alpha} \xi^{\alpha} \xi^{\beta}=0, \\
D_{i j} \overline{\mathcal{J}}_{\beta}^{\alpha} \xi^{\alpha} \xi^{\beta}= & \ddot{f} \delta_{1 i} \delta_{1 j}+\frac{\dot{f}}{\left|\nu_{0}\right|}\left(\delta_{i j}-\delta_{1 i} \delta_{1 j}\right) \\
& -2\left(1-\delta_{1 i}\right)\left(1-\delta_{1 j}\right)\left(1-\frac{\sin \left|\nu_{0}\right|}{\left|\nu_{0}\right| \cos \left|\nu_{0}\right|}\right) \xi^{i} \xi^{j} .
\end{aligned}
$$

Using the symmetry of $\overline{\mathcal{J}}$, we get the desired results.

Remark 4.2. - Let $\left(m_{0}, \nu_{0}\right) \in$ NoCut. Assume $x$ is the Fermi coordinate system associated to the geodesic $\exp _{m_{0}} s \nu_{0}$ and $v$ is the fiber coordinates of $T M \rightarrow M$ naturally associated to $x$. It is well-known

$$
p_{2}=\exp _{p_{1}}\left[-\operatorname{grad}_{p_{1}} c\left(\cdot, p_{2}\right)\right],
$$

whenever $p_{2} \in M$ is not cut point with respect to $p_{1} \in M$. Assume the points $p_{a}$ for $a=1,2$ are in the domain of the Fermi coordinate system and set $x=x\left(p_{1}\right)$. Suppose $m=p_{1}$ and $m_{2}=\exp _{m}(\nu)$ and denote $v=\left(v^{i}\right), v^{i}=$ $\mathrm{d} x^{i}(\nu)$. Differentiating (4.8) with respect to the coordinates $x$ at $x(m)$, we have

$$
D_{k} X^{i}(x, v, 1) \nabla_{j}^{k} c\left(m, \exp _{m} \nu\right)=\delta_{j}^{\nabla} X^{i}(x, v, 1),
$$

where $\delta_{j}^{\nabla} X^{i}=\partial_{j} X^{i}-\Gamma_{j l}^{p}(x) v^{l} D_{p} X^{i}$ is defined in an intrinsic way (see [7]).

In the following, all terms are evaluated at the point $(x, v)=(0,(\tau, 0))$. It is implicitly understood throughout the calculations.

The critical point condition. - By differentiating the test function with respect to $x^{i}$, the first derivative condition on critical point could be read as

$$
\left(\partial_{i} \mathcal{J}_{\beta}^{\alpha}+\nabla_{i}^{k} u D_{k} \mathcal{J}_{\beta}^{\alpha}\right)\left(\xi_{0}\right)_{\alpha}\left(\xi_{0}\right)^{\beta}=0 .
$$

Under the curvature assumption (1.3), by the Hessian comparison theorem we have $h\left(m_{0}, \xi_{0}\right)>0$. The components of $\xi_{0}$ are denoted by $\left(\xi_{0}\right)^{i}$, i.e., $\xi_{0}=\left(\xi_{0}\right)^{i} \partial_{i}$. 
Differentiating two times on the test function $h$ with respect to $x$, the second derivative condition on extrema can be read as follows

$$
0 \leqslant I_{2}+I I_{2}+I I I_{2}+I V_{2}+V_{2},
$$

where

$$
\begin{aligned}
I_{2}= & -\left\langle\mathcal{J} \xi_{0}, \xi_{0}\right\rangle \mathcal{F}_{j}^{i} g^{j l} \partial_{i l}^{2} g_{\alpha \beta}\left(\xi_{0}\right)^{\alpha}\left(\xi_{0}\right)^{\beta}+\mathcal{F}_{j}^{i} g^{j l} \partial_{i l}^{2} g_{\alpha k} \mathcal{J}_{\beta}^{k}\left(\xi_{0}\right)^{\alpha}\left(\xi_{0}\right)^{\beta}, \\
I I_{2}= & -\tau \mathcal{F}_{j}^{i} \partial^{j} \Gamma_{1 i}^{k} D_{k} \mathcal{J}_{\beta}^{\alpha}\left(\xi_{0}\right)_{\alpha}\left(\xi_{0}\right)^{\beta}+\mathcal{F}_{j}^{i} g^{j l} \partial_{i l}^{2} \mathcal{J}_{\beta}^{\alpha}\left(\xi_{0}\right)_{\alpha}\left(\xi_{0}\right)^{\beta} \\
I I I_{2}= & 2 \mathcal{F}_{j}^{i} \nabla_{k}^{j} u \partial_{i} D k \mathcal{J}_{\beta}^{\alpha}\left(\xi_{0}\right)_{\alpha}\left(\xi_{0}\right)^{\beta} \\
I V_{2}= & 2\left(1+\frac{1}{\tau^{2}}\left\langle\mathcal{J} \xi_{0}, \xi_{0}\right\rangle\right) \mathcal{F}_{j}^{i} \nabla_{i}^{\alpha} u \nabla_{\beta}^{j} u\left(\xi_{0}\right)_{\alpha}\left(\xi_{0}\right)^{\beta} \\
& +\mathcal{F}_{j}^{i} \nabla_{i}^{k} u \nabla_{l}^{j} u D_{k} D^{l} \mathcal{J}_{\beta}^{\alpha}\left(\xi_{0}\right)_{\alpha}\left(\xi_{0}\right)^{\beta} \\
V_{2}= & \mathcal{F}_{j}^{i} \partial^{j} \nabla_{i}^{k} u D_{k} \mathcal{J}_{\beta}^{\alpha}\left(\xi_{0}\right)_{\alpha}\left(\xi_{0}\right)^{\beta}
\end{aligned}
$$

Here $\left(\xi_{0}\right)_{i}=g_{i j}\left(\xi_{0}\right)^{j}$ are coordinates of the corresponding co-vector related to $\xi_{0}$. The potential function $u$ evaluated at the point $m_{0}$ satisfies the equation

$$
\operatorname{det} J_{0} \operatorname{det}\left(H_{j}^{i}\right)=\frac{\rho_{0}}{\rho_{t} \circ G} .
$$

with the matrix $\left(H_{j}^{i}\right)$ positive definite and $J_{0}=D_{v} X$. The positive definiteness of the matrix $\left(H_{j}^{i}\right)$ implies that $\nabla_{1}^{1} u$ is larger than -1 . We also write the expression

$$
S(0,(\tau, 0), 1)=\left[\begin{array}{cc}
1 & 0 \\
0 & S_{\beta}^{\alpha}
\end{array}\right] .
$$

Moreover, we have

$$
-S^{\perp} \geqslant-\frac{\tau \cos \tau}{\sin \tau} I_{n-1}
$$

We calculate each of the terms $I_{2}$ to $V_{2}$.

The term $I_{2}$. - Recall $g_{i j}=\delta_{i j}$ on the axis and $\mathcal{J}_{\alpha}^{1}=0$ at the point $(0,(\tau, 0))$ so that the term $I_{2}$ can be recast as

$$
I_{2}=-\left\langle\mathcal{J} \xi_{0}, \xi_{0}\right\rangle \mathcal{F}_{\psi}^{\varphi} g^{\psi \gamma} \partial_{\varphi \gamma}^{2} g_{\alpha \beta}\left(\xi_{0}\right)^{\alpha}\left(\xi_{0}\right)^{\beta}+\mathcal{F}_{\psi}^{\varphi} g^{\psi \gamma} \partial_{\varphi \gamma}^{2} g_{\alpha \iota} \mathcal{J}_{\beta}^{\iota}\left(\xi_{0}\right)^{\alpha}\left(\xi_{0}\right)^{\beta} .
$$

Then we calculate the term $I_{2}$ by Lemma 2.5 and find

$$
I_{2}=\frac{2}{3}\left\langle\mathcal{J} \xi_{0}, \xi_{0}\right\rangle \mathcal{F}_{\psi}^{\varphi} R_{\varphi \alpha}{ }_{\beta}{ }_{\beta}\left(\xi_{0}\right)^{\alpha}\left(\xi_{0}\right)^{\beta}-\frac{2}{3} \mathcal{F}_{\psi}^{\varphi} R_{\varphi \alpha}{ }_{\iota}{ }_{\iota} \mathcal{J}_{\beta}^{\iota}\left(\xi_{0}\right)^{\alpha}\left(\xi_{0}\right)^{\beta} .
$$

Using the curvature assumption (1.4) (or (1.5)), Proposition B.8, the positive definiteness of $\left(\mathcal{F}_{j}^{i}\right)$ and the Cauchy-Schwarz inequality, there exists a universal constant $C>0$ such that the following upper bound holds

$$
I_{2} \leqslant C \varepsilon \mathcal{F}_{\alpha}^{\alpha} .
$$


The term $\mathrm{II}_{2}$. - Using (1.4) (or (1.5)), Lemma 2.5, Proposition B.8 and the Cauchy-Schwarz inequality, there exists a universal constant $C>0$ (maybe different value than previous one) such that the following upper bound holds

$$
I I_{2} \leqslant C(\varepsilon+\delta) \mathcal{F}_{i}^{i} .
$$

Recalling $\mathcal{F}_{j}^{i} \nabla_{i}^{1} u \nabla_{1}^{j} u=\nabla_{1}^{1} u-1+\mathcal{F}_{1}^{1}$, we have $\mathcal{F}_{1}^{1} \leqslant 2+\mathcal{F}_{j}^{i} \nabla_{i}^{1} u \nabla_{1}^{j} u$ since $\nabla_{1}^{1} u \geqslant-1$. Thus, we infer

$$
I I_{2} \leqslant C(\varepsilon+\delta) \mathcal{F}_{\alpha}^{\alpha}+C(\varepsilon+\delta) \mathcal{F}_{j}^{i} \nabla_{i}^{k} u \nabla_{k}^{j} u+C .
$$

The term $I I I_{2}$. - Using $\mathcal{F}_{k}^{i} \nabla_{j}^{k} u=\delta_{j}^{i}-\mathcal{F}_{k}^{i} S_{j}^{k}$, we estimate

$$
I I I_{2}=2 \partial_{k} D^{k} \mathcal{J}_{\beta}^{\alpha}\left(\xi_{0}\right)_{\alpha}\left(\xi_{0}\right)^{\beta}+2 \mathcal{F}_{j}^{i} S_{k}^{j} \partial_{i} D k \mathcal{J}_{\beta}^{\alpha}\left(\xi_{0}\right)_{\alpha}\left(\xi_{0}\right)^{\beta} .
$$

From the Proposition B.8, there exists a universal constant $C>0$ such that

$$
I I I_{2} \leqslant C \varepsilon\left(1+\mathcal{F}_{j}^{i} S_{k}^{j} S_{i}^{k}\right) .
$$

Let us observe that the following identities hold: $\forall k, l=\{1, \ldots, n\}$

$$
\mathcal{F}_{j}^{i} \nabla_{i}^{k} u \nabla_{l}^{j} u=\nabla_{l}^{k} u-S_{l}^{k}+\mathcal{F}_{j}^{i} S_{i}^{k} S_{l}^{j}=\mathcal{H}_{l}^{k}-2 S_{l}^{k}+\mathcal{F}_{j}^{i} S_{i}^{k} S_{l}^{j} .
$$

The positive definiteness of $\left(\mathcal{H}_{j}^{i}\right),\left(-S^{\perp}\right)$ and the facts $S_{1}^{1}=1$ and $S_{\alpha}^{1}=0$ imply

$$
I I I_{2} \leqslant C \varepsilon\left(\mathcal{F}_{j}^{i} \nabla_{i}^{k} u \nabla_{k}^{j} u+1\right) .
$$

The term $I_{2}$. - Splitting the negative term

$$
\mathcal{F}_{j}^{i} \nabla_{i}^{k} u \nabla_{l}^{j} u D_{k} D^{l} \mathcal{J}_{\beta}^{\alpha}\left(\xi_{0}\right)_{\alpha}\left(\xi_{0}\right)^{\beta}
$$

into four parts, we have

$$
\begin{aligned}
I V_{2}= & \mathcal{F}_{j}^{i} \nabla_{i}^{1} u \nabla_{1}^{j} u D_{1} D^{1} \mathcal{J}_{\beta}^{\alpha}\left(\xi_{0}\right)_{\alpha}\left(\xi_{0}\right)^{\beta}+2 \mathcal{F}_{j}^{i} \nabla_{i}^{1} u \nabla_{\iota}^{j} u D_{1} D^{\iota} \mathcal{J}_{\beta}^{\alpha}\left(\xi_{0}\right)_{\alpha}\left(\xi_{0}\right)^{\beta} \\
& +\mathcal{F}_{j}^{i} \nabla_{i}^{\varphi} u \nabla_{\varphi}^{j} u D_{\varphi} D^{\varphi} \mathcal{J}_{\beta}^{\alpha}\left(\xi_{0}\right)_{\alpha}\left(\xi_{0}\right)^{\beta} \\
& +\sum_{\varphi \neq \psi} \mathcal{F}_{j}^{i} \nabla_{i}^{\varphi} u \nabla_{\psi}^{j} u D_{\varphi} D^{\psi} \mathcal{J}_{\beta}^{\alpha}\left(\xi_{0}\right)_{\alpha}\left(\xi_{0}\right)^{\beta} \\
& +2\left(1+\frac{1}{\tau^{2}}\left\langle\mathcal{J} \xi_{0}, \xi_{0}\right\rangle\right) \mathcal{F}_{j}^{i} \nabla_{i}^{\alpha} u \nabla_{\beta}^{j} u\left(\xi_{0}\right)_{\alpha}\left(\xi_{0}\right)^{\beta} .
\end{aligned}
$$

Using Proposition B.8, there exists a universal constant $C>0$ such that the following upper bound holds

$$
\begin{aligned}
I V_{2} \leqslant[C(\varepsilon+\delta)-2] \mathcal{F}_{j}^{i} \nabla_{i}^{1} u & \nabla_{1}^{j} u+[C(\varepsilon+\delta)-1] \mathcal{F}_{j}^{i} \nabla_{i}^{\alpha} u \nabla_{\alpha}^{j} u \\
+ & \frac{2}{\tau^{2}}\left\langle\mathcal{J} \xi_{0}, \xi_{0}\right\rangle \mathcal{F}_{j}^{i} \nabla_{i}^{\alpha} u \nabla_{\beta}^{j} u\left(\xi_{0}\right)_{\alpha}\left(\xi_{0}\right)^{\beta} \\
& -379-
\end{aligned}
$$


The term $V_{2}$. - The term $V_{2}$ involves the third derivatives of $u$. We use the equation (4.1). After commuting the third derivatives of $u$, the term $V_{2}$ can be estimated

$$
V_{2}=\mathcal{F}_{j}^{i}\left(\partial^{k} \nabla_{i}^{j} u+g^{j m} \tau R_{m 1 i}^{k}\right) D_{k} \mathcal{J}_{\beta}^{\alpha}\left(\xi_{0}\right)^{\alpha}\left(\xi_{0}\right)^{\beta} .
$$

By taking the logarithm and differentiating the equation (4.1) with respect to the variable $x^{k}$, we obtain the following simplified expressions

$$
\begin{aligned}
V_{2}= & {\left[\frac{\partial_{k} \rho_{0}}{\rho_{0}}-\frac{\partial_{l} \rho_{t}}{\rho_{t}}\left(J_{1}\right)_{k}^{l}-\left(J_{1}^{-1}\right)_{j}^{i} \partial_{k}\left(J_{1}\right)_{i}^{j}\right.} \\
& \left.\quad-\left(\mathcal{J}^{-1}\right)_{j}^{i} \partial_{k} \mathcal{J}_{i}^{j}-\mathcal{F}_{j}^{i} \partial_{k} S_{i}^{j}\right] D^{k} \mathcal{J}_{\beta}^{\alpha}\left(\xi_{0}\right)_{\alpha}\left(\xi_{0}\right)^{\beta} \\
+ & {\left[-\frac{\partial_{i} \rho_{t}}{\rho_{t}}\left(J_{0}\right)_{l}^{i}-\left(J_{1}^{-1}\right)_{j}^{i} D_{l}\left(J_{1}\right)_{i}^{j}\right.} \\
& \left.\quad-\left(\mathcal{J}^{-1}\right)_{j}^{i} D_{l} \mathcal{J}_{i}^{j}-\mathcal{F}_{j}^{i} D_{l} S_{i}^{j}\right] \nabla_{k}^{l} u D^{k} \mathcal{J}_{\beta}^{\alpha}\left(\xi_{0}\right)_{\alpha}\left(\xi_{0}\right)^{\beta} \\
+ & \frac{2 n}{\tau} \nabla_{k}^{1} u D^{k} \mathcal{J}_{\beta}^{\alpha}\left(\xi_{0}\right)_{\alpha}\left(\xi_{0}\right)^{\beta}+\tau g^{j m} \mathcal{F}_{j}^{i} R_{i 1 m}^{k} D_{k} \mathcal{J}_{\beta}^{\alpha}\left(\xi_{0}\right)_{\alpha}\left(\xi_{0}\right)^{\beta} .
\end{aligned}
$$

We observe that $\partial S=-S\left(\partial S^{-1}\right) S$ and $D S=-S\left(D S^{-1}\right) S$. Using the critical condition (4.10), Lemma A.4, Proposition B.8, the identities (4.15) and Cauchy-Schwarz inequality, there exists a positive constant $C>0$ such that there holds

$$
\begin{aligned}
V_{2} \leqslant C \max _{t \in[0,1]}\left\{\left|\operatorname{dlog} \rho_{t}\right|\right\}+C(\varepsilon+\delta) \mathcal{F}_{j}^{i} \nabla_{i}^{k} u \nabla_{k}^{j} u & \\
& +\left[C(\varepsilon+\delta)-\pi^{2}\right] \mathcal{F}_{\alpha}^{\alpha}+C .
\end{aligned}
$$

Gathering (4.11), (4.13), (4.14), (4.16), (4.17) and (4.18), we obtain the following inequality

$$
\begin{aligned}
0 \leqslant C \max _{t \in[0,1]}\left\{\left|\operatorname{dlog} \rho_{t}\right|\right\} & +\left[C(\varepsilon+\delta)-\pi^{2}\right] \mathcal{F}_{\alpha}^{\alpha}+[C(\varepsilon+\delta)-2] \mathcal{F}_{j}^{i} \nabla_{i}^{1} u \nabla_{1}^{j} u \\
& +\left[C(\varepsilon+\delta)+\frac{2}{\tau^{2}}\left\langle\mathcal{J} \xi_{0}, \xi_{0}\right\rangle-1\right] \mathcal{F}_{j}^{i} \nabla_{i}^{\alpha} u \nabla_{\alpha}^{j} u+C .
\end{aligned}
$$

Fix $\varepsilon<\frac{1}{8 C}, \delta<\frac{1}{8 C}$. Recalling (4.10), (4.12), (4.15), Proposition B.8 and using the fact the function $r \rightarrow-\frac{r \sin r}{\cos r}$ is decreasing in the interval $\left[\frac{3 \pi}{4}, \pi\right]$, we obtain the inequality

$$
\begin{aligned}
0 \leqslant C \max _{t \in[0,1]}\left\{\left|\operatorname{dlog} \rho_{t}\right|\right\}- & \frac{n-1}{2} \frac{(\pi-\delta) \cos \delta}{\sin \delta} \\
& +\left(\frac{2}{\tau^{2}}\left\langle\mathcal{J} \xi_{0}, \xi_{0}\right\rangle-\frac{1}{4}\right) \mathcal{F}_{j}^{i} \nabla_{i}^{\alpha} u \nabla_{\alpha}^{j} u+C .
\end{aligned}
$$


We take $\delta<\min \left\{\frac{1}{8 C}, \frac{3 \sqrt{2} \pi(n-1)}{16 C\left(\max _{t \in[0,1]}\left\{\left|\log \rho_{t}\right|\right\}+1\right)}\right\}$, the minimum $h\left(m_{0}, \xi_{0}\right)$ must be bounded below by

$$
\frac{1}{8}\left(\pi-\min \left\{\frac{1}{8 C}, \frac{3 \sqrt{2} \pi(n-1)}{16 C\left(\max _{t \in[0,1]}\left\{\left|\operatorname{dlog} \rho_{t}\right|\right\}+1\right)}\right\}\right)^{2} .
$$

Finally, the proof of Theorem 1.3 is complete.

\section{Appendix A. The geodesic motion and applications}

\section{A.1. The derivatives up to third order of geodesic motion}

In this subsection, we collect some results in [8]. Fix $m_{0} \in M, \nu_{0} \in$ $I\left(m_{0}\right) \backslash\{0\}$ and take the associated Fermi coordinate system along the geodesic $\exp _{m_{0}}\left(t \nu_{0}\right)$ and $v=\left(v^{1}, v^{2}, \ldots, v^{n}\right)$ be the fiber coordinates of $T M \rightarrow$ $M$ naturally associated to $x$. The curvatures of the Riemaniann manifold satisfy the assumptions (1.3) and (1.4) (or (1.5)). For $m \in M, \nu \in I(m)$ with $m$ in the domain of the Fermi coordinate system $x$, let $X(x, v, t)$ be the coordinates of the geodesic $\exp _{m} t \nu$. Then $X(x, v, t)$ is the solution of the Cauchy problem

$$
\ddot{X}^{k}+\Gamma_{i j}^{k}(X) \dot{X}^{i} \dot{X}^{j}=0, X^{k}(x, v, 0)=x^{k}, \dot{X}^{k}(x, v, 0)=v^{k} .
$$

In the Fermi coordinate system, $\left(m_{0}, \nu_{0}\right)$ corresponds to $\left(0, v_{0}\right)$ where $v_{0}=$ $\left(\left|\nu_{0}\right|, 0\right)$. On the axis, set for short $X_{0}(t):=X\left(0, v_{0}, t\right)$. We recall some results in [8]. Let $J_{0}=D_{v} X, J_{1}=\partial_{x} X$ be Jacobi fields in Section 2. Differentiating (A.1) once with respect to the variable $x$ (or $v$ ), on the axis, we get the following equation

$$
\ddot{J}_{a}^{i}+\partial_{l} \Gamma_{j k}^{i}(X) \dot{X}^{j} \dot{X}^{k} J_{a}^{l}+2 \Gamma_{j k}^{i}(X) \dot{X}^{j} \dot{J}_{a}^{k}=0,
$$

with the initial conditions, namely either

$$
\partial_{a} X^{i}(0)=\delta_{a}^{i}, \partial_{a} \dot{X}^{i}(0)=0
$$

or

$$
D_{a} X^{i}(0)=0, D_{a} \dot{X}^{i}(0)=\delta_{a}^{i} .
$$

We note that the equation (A.2) is equivalent to (2.5) on the axis. Thus, we have

Lemma A.1 ([8, Lemma 4]). — There exists a positive constant $C_{2}>0$ such that on the axis, for each $t \in[0,1]$, the terms

$$
\left|\partial_{x} X\left(0, v_{0}, t\right)\right|,\left|\partial_{x} \dot{X}\left(0, v_{0}, t\right)\right|,\left|D_{v} X\left(0, v_{0}, t\right)\right|,\left|D_{v} \dot{X}\left(0, v_{0}, t\right)\right| \text {, }
$$

are all bounded above by $C_{2}$. 
We require the notation $\overline{\partial_{x} X}(t)$ and $\overline{D_{v} X}(t)$ for the solution $\bar{J}_{a}$ of the unperturbed equation

$$
\ddot{\bar{J}}_{a}^{i}+\left|\nu_{0}\right|^{2} \bar{R}_{1 \beta 1}^{i} \bar{J}_{a}^{\beta}=0
$$

with the initial conditions, namely either

$$
\overline{\partial_{a} X^{i}}(0)=\delta_{a}^{i}, \overline{\partial_{a} X^{i}}(0)=0,
$$

or

$$
\overline{D_{a} X^{i}}(0)=0, \overline{D_{a} X^{i}}(0)=\delta_{a}^{i} .
$$

It is clear that $\overline{\partial_{x} X}(t)$ and $\overline{D_{v} X}(t)$ correspond to $\bar{J}_{1}$ and $\bar{J}_{0}$ respectively on the axis. Thus, we have

Lemma A.2 ([8, Lemma 5]). - There exists a positive constant $C_{3}>0$ such that on the axis, for each $t \in[0,1]$, we have

$$
\begin{aligned}
\left|\partial_{x} X\left(0, v_{0}, t\right)-\overline{\partial_{x} X}(t)\right| & \leqslant C_{3} \varepsilon, \\
\left|\partial_{x} \dot{X}\left(0, v_{0}, t\right)-\overline{\dot{\partial_{x} X}}(t)\right| & \leqslant C_{3} \varepsilon, \\
\left|D_{v} X\left(0, v_{0}, t\right)-\overline{D_{v} X}(t)\right| & \leqslant C_{3} \varepsilon, \\
\left|D_{v} \dot{X}\left(0, v_{0}, t\right)-\overline{D_{v} X}(t)\right| & \leqslant C_{3} \varepsilon .
\end{aligned}
$$

Remark A.3. - For later use, dealing with $\left|J_{0}-\bar{J}_{0}\right|$, the constant $C_{3}$ can be taken value $2 \sqrt{n-1}$ (see [8, Remark 5$]$ ).

Let $J_{a b}$ be $\partial_{a b}^{2} X, \partial_{a} D_{b} X, D_{a} \partial_{b} X$ or $D_{a b}^{2} X$. Differentiating the Cauchy problem (A.1) twice with respect to the parameters $x$ and $v$

$$
\begin{aligned}
\ddot{J}_{a b}^{i} & +\partial_{l} \Gamma_{j k}^{i} \dot{X}^{j} \dot{X}^{k} J_{a b}^{l}+2 \Gamma_{j k}^{i} \dot{X}^{j} \dot{J}_{a b}^{k} \\
& =-\partial_{l p}^{2} \Gamma_{j k}^{i} \dot{X}^{j} \dot{X}^{k} J_{a}^{l} J_{b}^{p}-2 \partial_{l} \Gamma_{j k}^{i} \dot{X}^{j}\left(\dot{J}_{b}^{k} J_{a}^{l}+\dot{J}_{a}^{k} J_{b}^{l}\right)-2 \Gamma_{j k}^{i} \dot{J}_{b}^{j} \dot{J}_{a}^{k} .
\end{aligned}
$$

and the homogenuous initial conditions

$$
J_{a b}^{i}(0)=\dot{J}_{a b}^{i}(0)=0 .
$$

On the axis, recalling (2.15), equation (A.3) is reduced to $\ddot{J}_{a b}^{i}+\left|\nu_{0}\right|^{2} R_{1 \alpha 1}^{i}\left(X_{0}\right) J_{a b}^{\alpha}=-\left|\nu_{0}\right|^{2} \partial_{l p}^{2} \Gamma_{11}^{i} J_{a}^{l} J_{b}^{p}-2\left|\nu_{0}\right| R_{k \beta 1}^{i}\left(X_{0}\right)\left(\dot{J}_{b}^{k} J_{a}^{\beta}+\dot{J}_{a}^{k} J_{b}^{\beta}\right)$. By Lemma 2.5, we have (see [8, Lemma 9])

Lemma A.4. - There exists a positive constant $C_{4}>0$ such that on the axis, for each $t \in[0,1]$, the terms

$$
\begin{array}{ll}
\left|\partial_{x x}^{2} X\left(0, v_{0}, t\right)\right|, & \left|\partial_{x x}^{2} \dot{X}\left(0, v_{0}, t\right)\right|, \\
\left|\partial_{x} D_{v} X\left(0, v_{0}, t\right)\right|, & \left|\partial_{x} D_{v} \dot{X}\left(0, v_{0}, t\right)\right|, \\
\left|D_{v v}^{2} X\left(m_{0}, v_{0}, t\right)\right|, & \left|D_{v v}^{2} \dot{X}\left(0, v_{0}, t\right)\right|
\end{array}
$$

are all bounded by $C_{4}$. 
Let us introduce the solutions $\overline{\partial_{x x}^{2} X}, \overline{\partial_{x} D_{v} X}, \overline{D_{v} \partial_{x} X}$ and $\overline{D_{v v}^{2} X}$ along the axis of the unperturbed equation

$$
\ddot{\bar{J}}_{a b}^{i}+\left|\nu_{0}\right|^{2} \delta_{\alpha}^{i} \bar{J}_{a b}^{\alpha}=-2\left|\nu_{0}\right|\left(\delta_{\beta}^{i} \delta_{k}^{1}-\delta_{1}^{i} \delta_{k}^{\beta}\right)\left(\bar{J}_{a}^{\beta} \dot{\bar{J}}_{b}^{k}+\bar{J}_{b}^{\beta} \dot{\bar{J}}_{a}^{k}\right),
$$

with null initial conditions

$$
\bar{J}_{a b}^{i}(0)=\dot{\bar{J}}_{a b}^{i}(0)=0 .
$$

We have the following perturbation result (see [8, Lemma 10]).

Lemma A.5. - There exists a positive constant $C_{5}>0$ such that on the axis, for each $t \in[0,1]$, we have

$$
\begin{aligned}
& \left|\partial_{x x}^{2} X\left(0, v_{0}, t\right)-\overline{\partial_{x x}^{2} X}(t)\right| \leqslant C_{5} \varepsilon \\
& \left|\partial_{x x}^{2} \dot{X}\left(0, v_{0}, t\right)-\overline{\partial_{x x}^{2} X}(t)\right| \leqslant C_{5} \varepsilon \\
& \left|\partial_{x} D_{v} X\left(0, v_{0}, t\right)-\overline{\partial_{x} D_{v} X}(t)\right| \leqslant C_{5} \varepsilon \\
& \left|\partial_{x} D_{v} \dot{X}\left(0, v_{0}, t\right)-\overline{\partial_{x} D_{v} X}(t)\right| \leqslant C_{5} \varepsilon \\
& \left|D_{v v}^{2} X\left(0, v_{0}, t\right)-\overline{D_{v v}^{2} X}(t)\right| \leqslant C_{5} \varepsilon \\
& \left|D_{v v}^{2} \dot{X}\left(0, v_{0}, t\right)-\overline{D_{v v}^{2} X}(t)\right| \leqslant C_{5} \varepsilon .
\end{aligned}
$$

Furthermore, $\left|\partial_{1 x}^{2} X\left(0, v_{0}, t\right)\right| \leqslant C_{5} \varepsilon,\left|\partial_{1} D_{v} X\left(0, v_{0}, t\right)\right| \leqslant C_{5} \varepsilon$.

Proof. - The first 6 statements are the results in (or comes directly from) [8, Lemma 10]. The last two approximations are just the consequences of the facts $\overline{\partial_{1 x}^{2} X}(t)=0$ and $\overline{\partial_{1} D_{v} X}(t)=0$ which follows from the equation (A.5) and the initial conditions (A.6).

Let $J_{a b c}^{i}(t)$ equal to $\partial_{x x x}^{3} X\left(0, v_{0}, t\right), \partial_{x x}^{2} D_{v} X\left(0, v_{0}, t\right), \partial_{x} D_{v v}^{2} X\left(0, v_{0}, t\right)$ or $D_{v v v}^{3} X\left(0, v_{0}, t\right)$. Differentiating (A.1) three times with respect to the variables $x$ and $v$

$$
\begin{aligned}
\ddot{J}_{a b c}^{i}+\left|\nu_{0}\right|^{2} R_{1 \alpha 1}^{i}\left(X_{0}\right) J_{a b c}^{\alpha}= & -\left|\nu_{0}\right|^{2} \partial_{l p q}^{3} \Gamma_{11}^{i} J_{a}^{l} J_{b}^{p} J_{c}^{q}-\left|\nu_{0}\right|^{2} \partial_{l p}^{2} \Gamma_{11}^{i} \sum_{(a, b, c)} J_{a b}^{l} J_{c}^{p} \\
& -2\left|\nu_{0}\right| \partial_{l p}^{2} \Gamma_{1 k}^{i} \sum_{(a, b, c)} \dot{J}_{a}^{k} J_{b}^{l} J_{c}^{p} \\
& -2\left|\nu_{0}\right| R_{k \beta 1}^{i}\left(X_{0}\right) \sum_{(a, b, c)}\left(\dot{J}_{a}^{k} J_{b c}^{\beta}+\dot{J}_{a b}^{k} J_{c}^{\beta}\right) \\
& -2 \partial_{\beta} \Gamma_{j k}^{i} \sum_{(a, b, c)} \dot{J}_{a}^{j} \dot{J}_{b}^{k} J_{c}^{\beta} .
\end{aligned}
$$

with null initial conditions

$$
J_{a b c}^{i}(0)=\dot{J}_{a b c}^{i}(0)=0 .
$$


Here $\sum_{(a, b, c)}$ means circular summation on $(a, b, c)$. It is stated (or with the same arguments) in [8, Lemma 12].

Lemma A.6. - There exists a positive constant $C_{6}>0$ such that on the axis, for each $t \in[0,1]$, the terms

$$
\begin{array}{ll}
\left|\partial_{x x x}^{3} X\left(0, v_{0}, t\right)\right|, & \left|\partial_{x x x}^{3} \dot{X}\left(0, v_{0}, t\right)\right|, \\
\left|\partial_{x x}^{2} D_{v} X\left(0, v_{0}, t\right)\right|, & \left|\partial_{x x}^{2} D_{v} \dot{X}\left(0, v_{0}, t\right)\right|, \\
\left|\partial_{x} D_{v v}^{2} X\left(0, v_{0}, t\right)\right|, & \left|\partial_{x} D_{v v}^{2} \dot{X}\left(0, v_{0}, t\right)\right|, \\
\left|D_{v v v}^{3} X\left(0, v_{0}, t\right)\right|, & \left|D_{v v v}^{3} \dot{X}\left(0, v_{0}, t\right)\right|
\end{array}
$$

are all bounded above a universal positive constant $C_{6}$.

Let us introduce the solutions $\overline{\partial_{x x x}^{3} X}, \overline{\partial_{x x}^{2} D_{v} X}, \overline{\partial_{x} D_{v} \partial_{x} X}, \overline{D_{v} \partial_{x x}^{2} X}$, $\overline{\partial_{x} D_{v v}^{2} X}, \overline{D_{v} \partial_{x} D_{v} X}, \overline{D_{v v}^{2} \partial_{x} X}$ and $\overline{D_{v v v}^{3} X}$ along the axis of the unperturbed equation

$$
\begin{aligned}
\ddot{\bar{J}}_{a b c}^{i} & +\left|\nu_{0}\right|^{2} \delta_{\alpha}^{i} \bar{J}_{a b c}^{\alpha} \\
= & \frac{4}{3}\left(\delta_{k}^{i}-\delta_{1}^{i} \delta_{k}^{1}\right) \sum_{(a, b, c)}\left(\left|\nu_{0}\right|^{2} \bar{J}_{a}^{k} \bar{J}_{b}^{\beta} \bar{J}_{c}^{\beta}-2 \dot{\bar{J}}_{a}^{\beta} \dot{\bar{J}}_{b}^{\beta} \bar{J}_{c}^{k}\right) \\
& -2\left|\nu_{0}\right|\left(\delta_{\beta}^{i} \delta_{k}^{1}-\delta_{1}^{i} \delta_{k}^{\beta}\right) \sum_{(a, b, c)}\left(\dot{\bar{J}}_{a}^{k} \bar{J}_{b c}^{\beta}+\dot{\bar{J}}_{a b}^{k} \bar{J}_{c}^{\beta}\right)-2\left(\delta_{k}^{i}-\delta_{1}^{i} \delta_{k}^{1}\right) \sum_{(a, b, c)} \dot{\bar{J}}_{a}^{1} \dot{\bar{J}}_{b}^{1} \bar{J}_{c}^{k} \\
& +\left[2 \delta_{1}^{i} \delta_{k}^{1}+\frac{2}{3}\left(\delta_{k}^{i}-\delta_{1}^{i} \delta_{k}^{1}\right)\right] \sum_{(a, b, c)}\left(\dot{\bar{J}}_{a}^{k} \dot{\bar{J}}_{b}^{\beta} \bar{J}_{c}^{\beta}+\dot{\bar{J}}_{a}^{\beta} \dot{\bar{J}}_{b}^{k} \bar{J}_{c}^{\beta}\right) .
\end{aligned}
$$

with null initial conditions

$$
\bar{J}_{a b c}^{i}(0)=\dot{\bar{J}}_{a b c}^{i}(0)=0 .
$$

It is shown (or with the same arguments) in [8, Lemma 13].

Lemma A.7. - There exists a positive constant $C_{7}>0$ such that on the axis, for each $t \in[0,1]$, we have

$$
\begin{aligned}
& \left|\partial_{x x x}^{3} X\left(0, v_{0}, t\right)-\overline{\partial_{x x}^{3} X}(t)\right| \leqslant C_{7} \varepsilon, \\
& \left|\partial_{x x}^{2} D_{v} X\left(0, v_{0}, t\right)-\overline{\partial_{x x}^{2} D_{v} X}(t)\right| \leqslant C_{7} \varepsilon, \\
& \left|\partial_{x} D_{v v}^{2} X\left(0, v_{0}, t\right)-\overline{\partial_{x} D_{v v}^{2} X}(t)\right| \leqslant C_{7} \varepsilon, \\
& \left|D_{v v v}^{3} X\left(0, v_{0}, t\right)-\overline{D_{v v v}^{3} X}(t)\right| \leqslant C_{7} \varepsilon .
\end{aligned}
$$




\section{Appendix B. The behaviour of Jacobi fields and applications}

\section{B.1. The behaviour of the curvature matrix}

In this subsection, we study the behaviour of the curvature matrix. Given $m_{0} \in M, \nu_{0} \in I\left(m_{0}\right) \backslash\{0\}$ and some orthonormal basis $\left\{E_{1}=\frac{\nu_{0}}{\left|\nu_{0}\right|}, E_{2}, \ldots, E_{n}\right\}$ of the tangent space $T_{m_{0}} M$, let $\gamma_{\theta}(t)$ be the geodesic with the initial point $m_{0}$ and the initial velocity $\cos \theta E_{1}+\sin \theta E_{2}$. For $|\theta|$ small enough, let $\left\{e_{1}(\theta, t), e_{2}(\theta, t), \ldots, e_{n}(\theta, t)\right\}$ be the parallel transport along the geodesic $\gamma_{\theta}(t)$ with $e_{1}(\theta, 0)=\cos \theta E_{1}+\sin \theta E_{2}, e_{2}(\theta, 0)=-\sin \theta E_{1}+\cos \theta E_{2}$, and $e_{i}(\theta, 0)=E_{i}$ for $i \geqslant 3$. Then $\left\{e_{1}(0, t), e_{2}(0, t), \ldots, e_{n}(0, t)\right\}$ is the parallel orthonormal moving frame along the geodesic $\gamma_{0}(t)$ with $e_{1}(0, t)=\dot{\gamma}_{0}(t)$.

Let $X(\theta, t)=\left(X^{1}(\theta, t), X^{2}(\theta, t), \ldots, X^{n}(\theta, t)\right)$ denote the coordinate of the geodesic $\gamma_{\theta}(t)$ in the Fermi coordinate system, i.e.

$$
X^{k}(\theta, t)=X^{k}(0,(\cos \theta, \sin \theta, 0, \ldots, 0), t) .
$$

Along the geodesic $\gamma_{\theta}(t)$, there are two bases in the tangent space: the natural basis $\left\{\frac{\partial}{\partial x^{i}}\right\}$ and the orthonormal basis $\left\{e_{1}, \ldots, e_{n}\right\}$. Set $\left(Y_{i}^{j}(\theta, t)\right)$ for the coordinates of the orthonormal chart $\left\{e_{1}, \ldots, e_{n}\right\}$, i.e. $e_{i}(\theta, t)=Y_{i}^{j}(\theta, t) \partial_{j}$. It is clear that $Y_{1}^{i}(\theta, t)=\dot{X}^{i}(\theta, t)$. Recall the dot stands for the derivative with respect to $t$ and the prime for the derivative with respect to $\theta$. Given two real function $f(t)$ and $h(t)$, we write $f(t)=\mathcal{B}(h(t))$ if there exists a positive constant $C$ (under control) such that $|f(t)| \leqslant C|h(t)|$ for all $t$ in a given range. The third derivative of $f(t)$ with respect to $t$ will be denoted by $\ddot{\ddot{f}}(t)$. The derivatives of the elements of the $n \times k$ matrix-valued function $\left(a_{j}^{i}(\theta, t)\right)$ with respect to $t$ and $\theta$ will be denoted by $\dot{a}_{j}^{i}(\theta, t), a_{j}^{i}(\theta, t), \ldots$, etc. For short, we drop the indices $x$ and $v$ if there is no confusion in the context.

\section{B.1.1. More asymptotic behaviour of the geodesic motion}

We investigate the geodesic motion. In particular, we study its asymptotic behaviour near the origin.

Lemma B.1. - Under the curvature assumptions (1.3) and (1.4) (or (1.5)) with $\varepsilon<1$, for all $t \in[0, \tau]$ with $\tau=\left|\nu_{0}\right|$, the geodesic motion on the axis satisfies in Fermi chart

(1) $X^{\prime}(0, t)=\left(0, \sin t+\mathcal{B}\left(\varepsilon t^{3}\right), \mathcal{B}\left(\varepsilon t^{3}\right), \ldots, \mathcal{B}\left(\varepsilon t^{3}\right)\right)^{T}$, $\dot{X}^{\prime}(0, t)=\left(0, \cos t+\mathcal{B}\left(\varepsilon t^{2}\right), \mathcal{B}\left(\varepsilon t^{2}\right), \ldots, \mathcal{B}\left(\varepsilon t^{2}\right)\right)^{T}$, $\ddot{X}^{\prime}(0, t)=(0,-\sin t+\mathcal{B}(\varepsilon t), \mathcal{B}(\varepsilon t), \ldots, \mathcal{B}(\varepsilon t))^{T}$, where ${ }^{T}$ is the transpose; 
(2) $X^{\prime \prime}(0, t)=\left(-\sin t \cos t+\mathcal{B}\left(\varepsilon t^{3}\right), \mathcal{B}\left(\varepsilon t^{3}\right), \ldots, \mathcal{B}\left(\varepsilon t^{3}\right)\right)^{T}$, $\dot{X}^{\prime \prime}(0, t)=\left(-\cos (2 t)+\mathcal{B}\left(\varepsilon t^{2}\right), \mathcal{B}\left(\varepsilon t^{2}\right), \ldots, \mathcal{B}\left(\varepsilon t^{2}\right)\right)^{T}$, $\ddot{X}^{\prime \prime}(0, t)=(4 \sin t \cos t+\mathcal{B}(\varepsilon t), \mathcal{B}(\varepsilon t), \ldots, \mathcal{B}(\varepsilon t))^{T}$

(3) $\ddot{X}^{\prime}(0,0)=\left(0,-R_{121}^{2}(0), \ldots,-R_{121}^{n}(0)\right)^{T}$, $\ddot{X}^{\prime \prime}(0,0)=\left(-4 R_{221}^{1}(0),-4 R_{221}^{2}(0), \ldots,-4 R_{221}^{n}(0)\right)^{T}$.

Proof. - The coordinates of the geodesic $\exp _{m_{0}} t\left(\cos \theta E_{1}+\sin \theta E_{2}\right)$ are the solution of the following Cauchy problem

$$
\left\{\begin{array}{l}
\ddot{X}^{i}+\Gamma_{j k}^{i}(X) \dot{X}^{j} \dot{X}^{k}=0, \\
X(\theta, 0)=0, \dot{X}(\theta, 0)=(\cos \theta, \sin \theta, 0, \ldots, 0)^{T} .
\end{array}\right.
$$

On the axis, since the Christoffel symbols vanish, we have

$$
X(0, t)=(t, 0, \ldots, 0)^{T} .
$$

(1). - Differentiating (B.1) with respect to $\theta$, we obtain

$$
\ddot{X}^{\prime i}+\partial_{p} \Gamma_{j k}^{i} \dot{X}^{j} \dot{X}^{k} X^{\prime p}+2 \Gamma_{j k}^{i} \dot{X}^{\prime j} \dot{X}^{k}=0,
$$

with the initial condition

$$
X^{\prime i}(0,0)=0, \dot{X}^{\prime i}(0,0)=\delta_{2}^{i} .
$$

It follows from (2.14) and (2.17) that on the axis we have

$$
\left\{\begin{array}{l}
\ddot{X}^{\prime}+R_{1 \alpha 1}^{i} X^{\prime \alpha}=0, \\
X^{\prime}(0,0)=0, \dot{X}^{\prime}(0,0)=(0,1,0, \ldots, 0)^{T} .
\end{array}\right.
$$

It is clear that $X^{\prime 1}(0, t) \equiv 0$. For $i>1$, we first establish the following standard estimation.

Claim. - For any $t \in[0, \tau] \subset[0, \pi]$,

$$
\max \left\{\left|X^{\prime}(0, t)\right|,\left|\dot{X}^{\prime}(0, t)\right|\right\} \leqslant e^{\frac{\pi}{2}} .
$$

Proof of the Claim. - Let $f=\left|X^{\prime}\right|^{2}+\left|\dot{X}^{\prime}\right|^{2}$ with $f(0)=1$. The derivative of $f$ has the form $\dot{f}=2\left(\bar{R}_{1 \alpha 1}^{\beta}(X)-R_{1 \alpha 1}^{\beta}(X)\right) X^{\prime \alpha} \dot{X}^{\prime \beta}$. Recall $\varepsilon \leqslant 1$. Using the Cauchy-Schwarz inequality, we get $\dot{f} \leqslant f$. We conclude that $f(t) \leqslant e^{t} \leqslant e^{\pi}$. Thus claim is proved.

We define $\overline{X^{\prime}}(t)=\left({\overline{X^{\prime}}}^{1}(t), \ldots,{\overline{X^{\prime}}}^{n}(t)\right)$ so that

$$
\left\{\begin{array}{l}
{\ddot{X^{\prime}}}^{i}+\bar{R}_{1 \alpha 1}^{i} \overline{X^{\prime}}{ }^{\alpha}=0, \\
\overline{X^{\prime}}(0,0)=0, \overline{X^{\prime}}(0,0)=(0,1,0, \ldots, 0)^{T} .
\end{array}\right.
$$

Denote $\mathcal{E}^{i}$ be the difference $X^{\prime i}-{\overline{X^{\prime}}}^{i}$. We rewrite the equation (B.2) in the perturbative form

$$
\ddot{\mathcal{E}}^{i}+\mathcal{E}^{i}=\left(\bar{R}_{1 \alpha 1}^{i}-R_{1 \alpha 1}^{i}\right) X^{\prime \alpha},
$$


with the homogeneous initial conditions

$$
\mathcal{E}^{i}(0,0)=\dot{\mathcal{E}}^{i}(0,0)=0 .
$$

By the representation formula (2.10), we get

$$
\begin{aligned}
\mathcal{E}^{i}(0, t)=\sin t \int_{0}^{t}\left(\bar{R}_{1 \alpha 1}^{i}-R_{1 \alpha 1}^{i}\right) X^{\prime \alpha} & \cos s \mathrm{~d} s \\
& \quad-\cos t \int_{0}^{t}\left(\bar{R}_{1 \alpha 1}^{i}-R_{1 \alpha 1}^{i}\right) X^{\prime \alpha} \sin s \mathrm{~d} s .
\end{aligned}
$$

which yields by the above claim $\left|\mathcal{E}^{i}(0, t)\right| \leqslant \frac{3}{2} \varepsilon t^{2} e^{\frac{\pi}{2}}$ for all $t \in[0, \tau]$. More precisely, we have for all $t \in[0, \tau]$

$$
\left|X^{\prime 2}(0, t)-\sin t\right| \leqslant \frac{3}{2} \varepsilon t^{2} e^{\frac{\pi}{2}}, \quad\left|X^{\prime j}(0, t)\right| \leqslant \frac{3}{2} \varepsilon t^{2} e^{\frac{\pi}{2}}, \quad \forall j \geqslant 3 .
$$

Using (B.3), we have $\left|\ddot{\mathcal{E}}^{i}(0, t)\right| \leqslant\left(\frac{9}{2} \pi e^{\frac{\pi}{2}}+1\right) \varepsilon t$. Therefore,

$$
\dot{\mathcal{E}}(0, t)=\int_{0}^{t} \ddot{\mathcal{E}}(0, s) \mathrm{d} s=\mathcal{B}\left(\varepsilon t^{2}\right), \quad \mathcal{E}(0, t)=\int_{0}^{t} \dot{\mathcal{E}}(0, s) \mathrm{d} s=\mathcal{B}\left(\varepsilon t^{3}\right) .
$$

Hence, we prove the first part.

(2). - Differentiating (B.1) twice with respect to $\theta$, we obtain

$$
\begin{aligned}
\ddot{X}^{\prime \prime i}+\partial_{p} \Gamma_{j k}^{i} \dot{X}^{j} \dot{X}^{k} X^{\prime \prime p}+\partial_{p q}^{2} \Gamma_{j k}^{i} \dot{X}^{j} \dot{X}^{k} X^{\prime p} X^{\prime q} \\
+4 \partial_{p} \Gamma_{j k}^{i} \dot{X}^{\prime j} \dot{X}^{k} X^{\prime p}+2 \Gamma_{j k}^{i}\left(\dot{X}^{\prime \prime j} \dot{X}^{k}+\dot{X}^{\prime j} \dot{X}^{\prime k}\right)=0,
\end{aligned}
$$

with the initial condition

$$
X^{\prime \prime}(0,0)=0, \quad \dot{X}^{\prime \prime}(0,0)=-\delta_{1}^{i} .
$$

Using (2.14), (2.16) and (2.17), we get on the axis

$$
\left\{\begin{array}{l}
\ddot{X}^{\prime \prime i}+R_{1 \alpha 1}^{i} X^{\prime \prime}+\left(\nabla_{\alpha} R_{1 \beta 1}^{i}+\nabla_{1} R_{\beta \alpha 1}^{i}\right) X^{\prime \alpha} X^{\prime \beta}+4 R_{\beta \alpha 1}^{i} X^{\prime \alpha} \dot{X}^{\prime \beta}=0, \\
X^{\prime \prime}(0,0)=0, \dot{X}^{\prime \prime}(0,0)=(-1,0, \ldots, 0)^{T} .
\end{array}\right.
$$

Claim. - There exists a positive universal constant $C$ such that, for any $t \in[0, \tau] \subset[0, \pi]$,

$$
\max \left\{\left|X^{\prime \prime}(0, t)\right|,\left|\dot{X}^{\prime \prime}(0, t)\right|\right\} \leqslant e^{C \pi} .
$$

Proof of the Claim. - Let $f_{1}(t)=\left|X^{\prime \prime}(0, t)\right|^{2}+\left|\dot{X}^{\prime \prime}(0, t)\right|^{2}$. Then

$$
\begin{aligned}
\dot{f}_{1}(t)= & 2 X^{\prime \prime i} \dot{X}^{\prime \prime i}+2 \dot{X}^{\prime \prime i} \ddot{X}^{\prime \prime} \\
= & 2 X^{\prime \prime} \dot{X}^{\prime \prime i}-2 R_{1 \alpha 1}^{i} X^{\prime \prime \alpha} \dot{X}^{\prime \prime i} \\
& -2\left[\left(\nabla_{\alpha} R_{1 \beta 1}^{i}+\nabla_{1} R_{\beta \alpha 1}^{i}\right) X^{\prime \alpha} X^{\prime \beta}+4 R_{\beta \alpha 1}^{i} X^{\prime \alpha} \dot{X}^{\prime \beta}\right] \dot{X}^{\prime \prime i} \\
\leqslant & C_{1} f_{1}(t)+C_{1},
\end{aligned}
$$

which gives $f_{1}(t) \leqslant C_{1} e^{C_{1} t} \leqslant e^{C \pi}$. Thus the claim is proved. 
When $i=1$, let $f_{2}(t)=X^{\prime \prime 1}(0, t)+\sin t \cos t$. Thus

$$
\begin{aligned}
\ddot{f}_{2}(t)= & -\nabla_{1} R_{\beta \alpha 1}^{1} X^{\prime \alpha} X^{\prime \beta}+4\left(\bar{R}_{\beta \alpha 1}^{1}-R_{\beta \alpha 1}^{1}\right) X^{\prime \alpha} \dot{X}^{\prime \beta} \\
& -4 \bar{R}_{\beta \alpha 1}^{1} X^{\prime \alpha} \dot{X}^{\prime \beta}-4 \sin t \cos t \\
= & 4{X^{\prime \alpha}}^{\prime \alpha} \dot{X}^{\prime \alpha}-4 \sin t \cos t+\mathcal{B}(\varepsilon t) \\
= & 4{X^{\prime 2}}^{\prime 2}-4 \sin t \cos t+\mathcal{B}(\varepsilon t) \\
= & 4\left(X^{\prime 2}-\sin t\right) \dot{X}^{\prime 2}+4\left(\dot{X}^{\prime 2}-\cos t\right) \sin t+\mathcal{B}(\varepsilon t) \\
= & \mathcal{B}(\varepsilon t) .
\end{aligned}
$$

When $i>1, X^{\prime \prime}$ satisfies the following equation

$$
\begin{array}{r}
\ddot{X}^{\prime \prime i}+X^{\prime \prime i}=\left(\bar{R}_{1 \alpha 1}^{i}-R_{1 \alpha 1}^{i}\right) X^{\prime \prime \alpha}-\left(\nabla_{\alpha} R_{1 \beta 1}^{i}+\nabla_{1} R_{\beta \alpha 1}^{i}\right) X^{\prime \alpha} X^{\prime \beta} \\
-4 R_{\beta \alpha 1}^{i} X^{\prime \alpha} \dot{X}^{\prime \beta},
\end{array}
$$

with the initial condition

$$
X^{\prime \prime}(0,0)=\dot{X}^{\prime \prime i}(0,0)=0 .
$$

By the representation formula (2.10), we get

$$
\begin{aligned}
X^{\prime \prime}(0, t)= & \sin t \int_{0}^{t}\left[\left(\bar{R}_{1 \alpha 1}^{i}-R_{1 \alpha 1}^{i}\right) X^{\prime \prime \alpha}-\left(\nabla_{\alpha} R_{1 \beta 1}^{i}+\nabla_{1} R_{\beta \alpha 1}^{i}\right) X^{\prime \alpha} X^{\prime \beta}\right. \\
& \left.-4 R_{\beta \alpha 1}^{i}{X^{\prime \alpha}}^{\alpha} \dot{X}^{\prime \beta}\right] \cos s \mathrm{~d} s \\
& -\cos t \int_{0}^{t}\left[\left(\bar{R}_{1 \alpha 1}^{i}-R_{1 \alpha 1}^{i}\right) X^{\prime \prime \alpha}-\left(\nabla_{\alpha} R_{1 \beta 1}^{i}+\nabla_{1} R_{\beta \alpha 1}^{i}\right) X^{\prime \alpha} X^{\prime \beta}\right. \\
& \left.-4 R_{\beta \alpha 1}^{i} X^{\prime \alpha} \dot{X}^{\prime \beta}\right] \sin s \mathrm{~d} s .
\end{aligned}
$$

Note that $\bar{R}_{1 \alpha 1}^{i}-R_{1 \alpha 1}^{i}=\mathcal{B}(\varepsilon), \nabla_{\alpha} R_{1 \beta 1}^{i}=\mathcal{B}(\varepsilon), \nabla_{1} R_{\beta \alpha 1}^{i}=\mathcal{B}(\varepsilon), R_{\beta \alpha 1}^{i}=$ $\mathcal{B}(\varepsilon)$ and $X^{\prime}, \dot{X}^{\prime}, X^{\prime \prime}$ are uniformly bounded shown by two previous claims. Thus we have $X^{\prime \prime}(0, t)=\mathcal{B}(\varepsilon t)$. Gathering the above results, we infer

$$
\ddot{X}^{\prime \prime}(0, t)=(4 \sin t \cos t, 0, \ldots, 0)^{T}+\mathcal{B}(\varepsilon t) .
$$

Integrating this equality, we obtain

$$
\begin{aligned}
& \dot{X}^{\prime \prime}(0, t)=(-\cos (2 t), 0, \ldots, 0)^{T}+\mathcal{B}\left(\varepsilon t^{2}\right), \\
& X^{\prime \prime}(0, t)=(-\sin t \cos t, 0, \ldots, 0)^{T}+\mathcal{B}\left(\varepsilon t^{3}\right) .
\end{aligned}
$$

(3). - Differentiating (B.1) once with respect to $\theta$ and $t$ respectively, we have

$$
\begin{array}{r}
\ddot{X}^{\prime i}+\partial_{p q}^{2} \Gamma_{j k}^{i} \dot{X}^{j} \dot{X}^{k} \dot{X}^{p} X^{\prime q}+\partial_{p} \Gamma_{j k}^{i}\left(\ddot{X}^{j} \dot{X}^{k} X^{\prime p}+\dot{X}^{j} \ddot{X}^{k} X^{\prime p}+\dot{X}^{j} \dot{X}^{k} \dot{X}^{\prime p}\right) \\
+2 \partial_{p} \Gamma_{j k}^{i} \dot{X}^{\prime j} \dot{X}^{k} \dot{X}^{p}+2 \Gamma_{j k}^{i}\left(\ddot{X}^{\prime j} \dot{X}^{k}+\dot{X}^{\prime j} \ddot{X}^{k}\right)=0 .
\end{array}
$$


Using the facts $\dot{X}(0,0)=(1,0, \ldots, 0)^{T}, X^{\prime}(0,0)=0, \dot{X}^{\prime}(0,0)=(0,1,0, \ldots, 0)^{T}$ and $\partial_{1} \Gamma_{i j}^{k}(0)=0$ and together with $(2.17)$, we get

$$
\ddot{X}^{\prime i}(0,0)=-R_{121}^{i}(0) \text {. }
$$

Differentiating (B.1) twice with respect to $\theta$ and once with respect to $t$ respectively

$$
\begin{aligned}
& \ddot{X}^{\prime \prime}{ }^{i}+\partial_{p q}^{2} \Gamma_{j k}^{i} \dot{X}^{j} \dot{X}^{k} \dot{X}^{p} X^{\prime \prime q}+\partial_{p} \Gamma_{j k}^{i}\left(2 \ddot{X}^{j} \dot{X}^{k} X^{\prime \prime p}+\dot{X}^{j} \dot{X}^{k} \dot{X}^{\prime \prime p}\right) \\
& +\partial_{p q l}^{3} \Gamma_{j k}^{i} \dot{X}^{j} \dot{X}^{k} X^{\prime p} X^{\prime q} \dot{X}^{l}+2 \partial_{p q}^{2} \Gamma_{j k}^{i}\left(\ddot{X}^{j} \dot{X}^{k} X^{\prime p} X^{\prime q}+\dot{X}^{j} \dot{X}^{k} \dot{X}^{\prime p} X^{\prime q}\right) \\
& +4 \partial_{p q}^{2} \Gamma_{j k}^{i} \dot{X}^{\prime j} \dot{X}^{k} \dot{X}^{p} X^{\prime q}+4 \partial_{p} \Gamma_{j k}^{i}\left(\ddot{X}^{\prime j} \dot{X}^{k} X^{\prime p}+\dot{X}^{\prime j} \ddot{X}^{k} X^{\prime p}+\dot{X}^{\prime j} \dot{X}^{k} \dot{X}^{\prime p}\right) \\
& +2 \partial_{p} \Gamma_{j k}^{i}\left(\dot{X}^{\prime \prime j} \dot{X}^{k}+\dot{X}^{\prime j} \dot{X}^{\prime k}\right) \dot{X}^{p}+2 \Gamma_{j k}^{i}\left(\ddot{X}^{\prime \prime} \dot{X}^{k}+\dot{X}^{\prime \prime j} \ddot{X}^{k}+2 \ddot{X}^{\prime j} \dot{X}^{\prime k}\right)=0 .
\end{aligned}
$$

Again by (2.17) and together with the facts $\dot{X}(0,0)=(1,0, \ldots, 0)^{T}, X^{\prime}(0,0)=$ $X^{\prime \prime}(0,0)=(0, \ldots, 0)^{T}, \dot{X}^{\prime}(0,0)=(0,1,0 \ldots, 0)^{T}, \dot{X}^{\prime \prime}(0,0)=(-1,0 \ldots, 0)^{T}$ and $\Gamma_{i j}^{k}(0)=\partial_{1} \Gamma_{i j}^{k}(0)=0$, we get

$$
\ddot{X}^{\prime \prime} i(0,0)=-4 R_{221}^{i}(0) \text {. }
$$

Finally, the Lemma is proved.

\section{B.1.2. The orthonormal basis motion}

We write $e_{i}(\theta, t)=Y_{i}^{j}(\theta, t) \partial_{j}$ for all $i$. Since the orthonormal moving basis $\left\{e_{1}, \ldots, e_{n}\right\}$ is parallel, we consider its equation in Fermi chart

$$
\dot{Y}_{j}^{i}+\Gamma_{k l}^{i}(X) \dot{X}^{l} Y_{j}^{k}=0,
$$

with the initial condition

$$
Y(\theta, 0)=\left[\begin{array}{ccc}
\cos \theta & -\sin \theta & \\
\sin \theta & \cos \theta & \\
& & I_{n-2}
\end{array}\right] .
$$

We remark when $j$ is equal to $1, Y_{1}^{i}(\theta, t)=\dot{X}^{i}(\theta, t)$. It is obvious that $Y(0, t)=I_{n}$

Lemma B.2. - Under the curvature assumptions (1.3) and (1.4) (or (1.5)) with $\varepsilon<1$, then in the Fermi chart, the derivatives of the basis motion along the axis satisfy the following estimates, $\forall t \in[0, \tau]$ with $\tau=\left|\nu_{0}\right|$

(1) $Y^{\prime i}{ }_{j}^{i}(0, t)=-\left(\delta_{1}^{i} \delta_{j}^{2}-\delta_{2}^{i} \delta_{j}^{1}\right) \cos t+\mathcal{B}\left(\varepsilon t^{2}\right)$,

$$
\dot{Y}^{\prime}{ }_{j}^{i}(0, t)=\left(\delta_{1}^{i} \delta_{j}^{2}-\delta_{2}^{i} \delta_{j}^{1}\right) \sin t+\mathcal{B}(\varepsilon t) ;
$$


(2) $Y_{j}^{\prime \prime}{ }_{j}^{i}(0, t)=-\delta_{1}^{i} \delta_{j}^{1} \cos (2 t)-\delta_{2}^{i} \delta_{j}^{2} \cos ^{2} t$

$$
\begin{gathered}
+\frac{1}{3}\left(\delta_{j}^{i}-\delta_{2}^{i} \delta_{j}^{2}-\delta_{1}^{j} \delta_{j}^{i}\right) \sin ^{2} t+\mathcal{B}\left(\varepsilon t^{2}\right), \\
\dot{Y}^{\prime \prime}{ }_{j}^{i}(0, t)=4 \\
4 \delta_{1}^{i} \delta_{j}^{1} \sin t \cos t+2 \delta_{2}^{i} \delta_{j}^{2} \sin t \cos t \\
+\frac{2}{3}\left(\delta_{j}^{i}-\delta_{2}^{i} \delta_{j}^{2}-\delta_{1}^{j} \delta_{j}^{i}\right) \sin t \cos t+\mathcal{B}(\varepsilon t) ;
\end{gathered}
$$

(3) $\dot{Y^{\prime \prime}}{ }_{j}^{i}(0,0)=0$,

$$
\ddot{Y}^{\prime \prime}{ }_{j}^{i}(0,0)=2 \delta_{j}^{2} R_{121}^{i}(0)-\frac{2}{3}\left(1+5 \delta_{j}^{1}\right) R_{22 j}^{i}(0) .
$$

Proof. -

(1). - Differentiating equation (B.4) with respect to $\theta$, we get

$$
\dot{Y}^{\prime}{ }_{j}^{i}+\partial_{p} \Gamma_{k l}^{i} \dot{X}^{l} X^{\prime p} Y_{j}^{k}+\Gamma_{k l}^{i}\left(\dot{X}^{\prime l} Y_{j}^{k}+\dot{X}^{l} Y_{j}^{\prime k}\right)=0
$$

which implies by Lemma 2.5

$$
\dot{Y}^{\prime}{ }_{j}^{i}+R_{j \alpha 1}^{i} X^{\prime \alpha}=0
$$

with initial condition

$$
Y_{j}^{\prime i}(0,0)=\delta_{2}^{i} \delta_{j}^{1}-\delta_{1}^{i} \delta_{j}^{2}
$$

Thus, we infer

$$
\begin{aligned}
\dot{Y}^{\prime}{ }_{j}^{i}(0, t) & =-R_{j \alpha 1}^{i} X^{\prime \alpha} \\
& =\left(\bar{R}_{j \alpha 1}^{i}-R_{j \alpha 1}^{i}\right) X^{\prime \alpha}-\bar{R}_{j \alpha 1}^{i} X^{\prime \alpha}
\end{aligned}
$$

By Lemma B.1, we have

$$
\begin{aligned}
\dot{Y}^{\prime}{ }_{j}^{i}(0, t) & =-\bar{R}_{j \alpha 1}^{i} X^{\prime \alpha}+\mathcal{B}(\varepsilon t) \\
& =-\bar{R}_{j 21}^{i} X^{\prime 2}+\mathcal{B}(\varepsilon t) \\
& =\left(\delta_{1}^{i} \delta_{j}^{2}-\delta_{2}^{i} \delta_{j}^{1}\right) \sin t+\mathcal{B}(\varepsilon t) .
\end{aligned}
$$

Integrating this equality, we deduce

$$
\begin{aligned}
Y_{j}^{\prime}(0, t) & =Y_{j}^{\prime}{ }_{j}^{i}(0,0)+\int_{0}^{t} \dot{Y}^{\prime}{ }_{j}^{i}(0, s) \mathrm{d} s \\
& =\delta_{2}^{i} \delta_{j}^{1}-\delta_{1}^{i} \delta_{j}^{2}+\left(\delta_{1}^{i} \delta_{j}^{2}-\delta_{2}^{i} \delta_{j}^{1}\right) \int_{0}^{t} \sin s \mathrm{~d} s+\mathcal{B}\left(\varepsilon t^{2}\right) \\
& =\left(\delta_{2}^{i} \delta_{j}^{1}-\delta_{1}^{i} \delta_{j}^{2}\right) \cos t+\mathcal{B}\left(\varepsilon t^{2}\right) .
\end{aligned}
$$

(2). - Differentiating equation (B.4) twice with respect to the parameter $\theta$, we obtain

$$
\begin{aligned}
\dot{Y}^{\prime \prime}{ }_{j}^{i}+2 & \partial_{p} \Gamma_{k l}^{i} \dot{X}^{l} X^{\prime p} Y_{j}^{\prime k}+\partial_{p} \Gamma_{k l}^{i} \dot{X}^{l} X^{\prime \prime p} Y_{j}^{k}+2 \partial_{p} \Gamma_{k l}^{i} \dot{X}^{\prime l} X^{\prime p} Y_{j}^{k} \\
& +\partial_{p q}^{2} \Gamma_{k l}^{i} \dot{X}^{l} X^{\prime p} X^{\prime q} Y_{j}^{k}+\Gamma_{k l}^{i}\left(\dot{X}^{\prime \prime l} Y_{j}^{k}+2 \dot{X}^{\prime l} Y_{j}^{\prime k}+\dot{X}^{l} Y_{j}^{\prime \prime}{ }_{j}^{k}\right)=0
\end{aligned}
$$


with the initial condition

$$
Y_{j}^{\prime \prime}(0,0)=-\delta_{1}^{i} \delta_{j}^{1}-\delta_{2}^{i} \delta_{j}^{2}
$$

which yields on the axis

$$
\dot{Y}^{\prime \prime \prime}{ }_{j}^{i}+2 R_{k \alpha 1}^{i} X^{\prime \alpha} Y_{j}^{\prime k}+R_{j \alpha 1}^{i} X^{\prime \prime \alpha}+2 \partial_{\alpha} \Gamma_{\beta j}^{i} X^{\prime \alpha} \dot{X}^{\prime \beta}+\partial_{\alpha \beta}^{2} \Gamma_{1 j}^{i} X^{\prime \alpha} X^{\prime \beta}=0,
$$

We consider the cases $j>1$. Using (2.16), (2.17) and Lemma B.1, we get

$$
\begin{aligned}
\dot{Y}^{\prime \prime}{ }_{j}^{i} & =-2 R_{k \alpha 1}^{i} X^{\prime \alpha} Y_{j}^{\prime k}-\frac{2}{3}\left(R_{\beta \alpha j}^{i}+R_{j \alpha \beta}^{i}\right) X^{\prime \alpha} \dot{X}^{\prime \beta}+\mathcal{B}(\varepsilon t) \\
& =-2 \bar{R}_{k \alpha 1}^{i} X^{\prime \alpha}{Y^{\prime}}_{j}^{k}-\frac{2}{3}\left(\bar{R}_{\beta \alpha j}^{i}+\bar{R}_{j \alpha \beta}^{i}\right) X^{\prime \alpha} \dot{X}^{\prime \beta}+\mathcal{B}(\varepsilon t) \\
& =-2 \delta_{j}^{2} \bar{R}_{121}^{i} X^{2^{\prime}} Y_{2}^{1^{\prime}}-\frac{2}{3}\left(\bar{R}_{22 j}^{i}+\bar{R}_{j 22}^{i}\right) X^{\prime 2} \dot{X}^{\prime 2}+\mathcal{B}(\varepsilon t) \\
& =2 \delta_{j}^{2} \bar{R}_{121}^{i} \sin t \cos t-\frac{2}{3} \bar{R}_{22 j}^{i} \sin t \cos t+\mathcal{B}(\varepsilon t) \\
& =2 \delta_{2}^{i} \delta_{j}^{2} \sin t \cos t+\frac{2}{3}\left(\delta_{j}^{i}-\delta_{2}^{i} \delta_{j}^{2}\right) \sin t \cos t+\mathcal{B}(\varepsilon t) .
\end{aligned}
$$

which gives by integration

$$
\begin{aligned}
Y_{j}^{\prime \prime}(0, t)= & Y_{j}^{\prime \prime}{ }_{j}^{i}(0,0)+\int_{0}^{t} \dot{Y}^{\prime \prime}{ }_{j}^{i}(0, s) \mathrm{d} s \\
= & -\delta_{2}^{i} \delta_{j}^{2}+2 \delta_{2}^{i} \delta_{j}^{2} \int_{0}^{t} \sin s \cos s \mathrm{~d} s \\
& +\frac{2}{3}\left(\delta_{j}^{i}-\delta_{2}^{i} \delta_{j}^{2}\right) \int_{0}^{t} \sin s \cos s \mathrm{~d} s+\mathcal{B}\left(\varepsilon t^{2}\right) \\
= & -\delta_{2}^{i} \delta_{j}^{2}+\delta_{2}^{i} \delta_{j}^{2} \sin ^{2} t+\frac{1}{3}\left(\delta_{j}^{i}-\delta_{2}^{i} \delta_{j}^{2}\right) \sin ^{2} t+\mathcal{B}\left(\varepsilon t^{2}\right) \\
= & -\delta_{2}^{i} \delta_{j}^{2} \cos ^{2} t+\frac{1}{3}\left(\delta_{j}^{i}-\delta_{2}^{i} \delta_{j}^{2}\right) \sin ^{2} t+\mathcal{B}\left(\varepsilon t^{2}\right) .
\end{aligned}
$$

Similarly, when $j=1$, it follows from Lemmas 2.5 and B.1

$$
\begin{aligned}
\dot{Y}^{\prime \prime}{ }_{1}^{i} & =-2 R_{k \alpha 1}^{i} X^{\prime \alpha} Y_{j}^{\prime k}-R_{1 \alpha 1}^{i} X^{\prime \prime \alpha}-2 \partial_{\alpha} \Gamma_{\beta 1}^{i} X^{\prime \alpha} \dot{X}^{\prime \beta}+\partial_{\alpha \beta}^{2} \Gamma_{11}^{i} X^{\prime \alpha} X^{\prime \beta} \\
& =-4 R_{221}^{i} \sin t \cos t+\mathcal{B}\left(\varepsilon t^{2}\right) \\
& =-4 R_{221}^{i}(0) \sin t \cos t+\mathcal{B}(\varepsilon t) \\
& =4 \delta_{1}^{i} \sin t \cos t+\mathcal{B}(\varepsilon t)
\end{aligned}
$$

Recalling the initial condition $Y_{1}^{\prime \prime}{ }_{1}^{i}(0,0)=-\delta_{1}^{i}$, this yields

$$
Y^{\prime \prime}{ }_{1}^{i}(0, t)=-\cos 2 t \delta_{1}^{i}+\mathcal{B}\left(\varepsilon t^{2}\right) .
$$

We prove (2). 
(3). - The first part comes directly (2).

For the second part, differentiating equation (B.4) twice with respect to $\theta$ and once with respect to $t$ respectively

$$
\begin{aligned}
& \ddot{Y}^{\prime \prime}{ }_{j}^{i}+2 \partial_{p q}^{2} \Gamma_{k l}^{i} \dot{X}^{l} \dot{X}^{p} X^{\prime q} Y_{j}^{\prime k}+2 \partial_{p} \Gamma_{k l}^{i}\left(\ddot{X}^{l} X^{\prime p} Y_{j}^{\prime k}+\dot{X}^{l} \dot{X}^{\prime p} Y_{j}^{\prime k}+\dot{X}^{l} X^{\prime p} \dot{Y}_{j}{ }_{j}^{k}\right) \\
& +\partial_{p q}^{2} \Gamma_{k l}^{i} \dot{X}^{l} \dot{X}^{p} X^{\prime \prime} Y_{j}^{k}+\partial_{p} \Gamma_{k l}^{i}\left(\ddot{X}^{l} X^{\prime \prime} Y_{j}^{k}+\dot{X}^{l} \dot{X}^{\prime \prime p} Y_{j}^{k}+\dot{X}^{l} X^{\prime \prime} \dot{Y}_{j}^{k}\right) \\
& +2 \partial_{p q}^{2} \Gamma_{k l}^{i} \dot{X}^{\prime l} \dot{X}^{p} X^{\prime q} Y_{j}^{k}+2 \partial_{p} \Gamma_{k l}^{i}\left(\ddot{X}^{\prime l} X^{\prime p} Y_{j}^{k}+\dot{X}^{\prime l} \dot{X}^{\prime p} Y_{j}^{k}+\dot{X}^{\prime l} X^{\prime p} \dot{Y}_{j}^{k}\right) \\
& +\partial_{a p q}^{3} \Gamma_{k l}^{i} \dot{X}^{l} \dot{X}^{a} X^{\prime p} X^{\prime q} Y_{j}^{k} \\
& +\partial_{p q}^{2} \Gamma_{k l}^{i}\left(\ddot{X}^{l} X^{\prime p} X^{\prime q} Y_{j}^{k}+\dot{X}^{l} \dot{X}^{\prime p} X^{\prime q} Y_{j}^{k}+\dot{X}^{l} X^{\prime p} \dot{X}^{\prime q} Y_{j}^{k}+\dot{X}^{l} X^{\prime p} X^{\prime q} \dot{Y}_{j}^{k}\right) \\
& +\partial_{p} \Gamma_{k l}^{i}\left(\dot{X}^{\prime \prime l} Y_{j}^{k}+2 \dot{X}^{\prime l} Y_{j}^{\prime k}+\dot{X}^{l} Y_{j}^{\prime \prime}{ }_{j}^{k}\right) \dot{X}^{p} \\
& +\Gamma_{k l}^{i}\left(\ddot{X}^{\prime \prime}{ }^{l} Y_{j}^{k}+\dot{X}^{\prime \prime l} \dot{Y}_{j}^{k}+2 \ddot{X}^{\prime \prime} Y_{j}^{\prime k}+2 \dot{X}^{\prime l} \dot{Y}_{j}^{\prime k}+\ddot{X}_{j}^{l} Y_{j}^{\prime \prime}{ }_{j}^{k}+\dot{X}^{l} \dot{Y}^{\prime \prime}{ }_{j}^{k}\right)=0,
\end{aligned}
$$

Combining (2.17) with the relations $\dot{X}(0,0)=(1,0, \ldots, 0)^{T}, X^{\prime}(0,0)=$ $X^{\prime \prime}(0,0)=(0, \ldots, 0)^{T}, \dot{X}^{\prime}(0,0)=(0,1,0, \ldots, 0)^{T}, \dot{X}^{\prime \prime}(0,0)=(-1,0, \ldots, 0)^{T}$, $Y(0,0)=I_{n}$ and $\Gamma_{i j}^{k}(0)=\partial_{1} \Gamma_{i j}^{k}(0)=0$, we get

$$
\begin{aligned}
\ddot{Y}^{\prime \prime}{ }_{j}^{i}(0,0) & =-2 R_{k 21}^{i}(0) Y_{j}^{\prime k}(0,0)-2 \partial_{2} \Gamma_{2 j}^{i}(0) \\
& =-2 \delta_{j}^{1} R_{221}^{i}(0)+2 \delta_{j}^{2} R_{121}^{i}(0)-2 \delta_{j}^{1} R_{221}^{i}(0)-\frac{2}{3}\left(1-\delta_{j}^{1}\right) R_{22 j}^{i}(0) \\
& =-\frac{10}{3} \delta_{j}^{1} R_{221}^{i}(0)+2 \delta_{j}^{2} R_{121}^{i}(0)-\frac{2}{3} R_{22 j}^{i}(0) \\
& =2 \delta_{j}^{2} R_{121}^{i}(0)-\frac{2}{3}\left(1+5 \delta_{j}^{1}\right) R_{22 j}^{i}(0) .
\end{aligned}
$$

We complete the proof of Lemma B.2.

\section{B.1.3. The behaviour of curvature matrix}

In this subsection, we take account of the derivatives of the curvature matrix. Combining (2.6) and equality $\dot{\gamma}_{\theta}(t)=e_{1}(\theta, t)$, by the anti-symmetry of the Riemann curvature tensor, $R_{i j}(\theta, t)=0$ when $i=1$ or $j=1$. Our main result in this subsection can be read as

Proposition B.3. - Under the curvature assumptions (1.3) and (1.4) (or (1.5)) with $\varepsilon<1$, then in the Fermi chart, we have on the axis: for all $t \in[0, \tau]$ with $\tau=\left|\nu_{0}\right|$

$$
\text { (1) } \begin{aligned}
\dot{R}_{i j}(0, t)= & \nabla_{1} R_{1 i 1 j}(X(0, t)) \\
R_{i j}^{\prime}(0, t)= & \left(R_{1 i 2 j}+R_{1 j 2 i}+R_{121 j} \delta_{i}^{1}+R_{121 i} \delta_{j}^{1}\right) \cos t \\
& \quad+\nabla_{2} R_{1 i 1 j} \sin t+\mathcal{B}\left(\varepsilon t^{2}\right) ;
\end{aligned}
$$


(2) When $i, j>1$,

$$
\begin{aligned}
& \ddot{R}_{i j}(0, t)=\nabla_{11}^{2} R_{1 i 1 j}(X(0, t)) \text {, } \\
& \dot{R}_{i j}^{\prime}(0, t)=\left(\nabla_{1} R_{1 i 2 j}+\nabla_{1} R_{1 j 2 i}+\nabla_{2} R_{1 i 1 j}\right) \cos t+\mathcal{B}(\varepsilon t), \\
& R_{i j}^{\prime \prime}(0, t)=\left[2 R_{2 i 2 j}-2 R_{1 i 1 j}+\delta_{i}^{2} R_{121 j}+\delta_{j}^{2} R_{121 i}+2 \delta_{i}^{1} R_{122 j}\right. \\
& \left.+2 \delta_{j}^{1} R_{122 i}+2 R_{1212} \delta_{i}^{1} \delta_{j}^{1}\right] \cos ^{2} t \\
& +\left(-\nabla_{1} R_{1 i 1 j}+2 \nabla_{2} R_{2 i 1 j}+2 \nabla_{2} R_{2 j 1 i}+2 \nabla_{2} R_{121 i} \delta_{j}^{1}\right. \\
& \left.+2 \nabla_{2} R_{121 j} \delta_{i}^{1}\right) \sin t \cos t+\mathcal{B}\left(\varepsilon t^{2}\right)
\end{aligned}
$$

(3) When $i, j>1$,

$$
\begin{aligned}
\dot{R}_{i j}^{\prime \prime}(0,0)=2 & \nabla_{1} R_{2 i 2 j}(0)-3 \nabla_{1} R_{1 i 1 j}(0)+\delta_{i}^{2} \nabla_{1} R_{121 j(0)} \\
& +\delta_{j}^{2} \nabla_{1} R_{121 i}(0)+2 \delta_{i}^{1} \nabla_{1} R_{122 j}(0)+2 \delta_{j}^{1} \nabla_{1} R_{122 i}(0) \\
& +2 \nabla_{1} R_{1212}(0) \delta_{i}^{1} \delta_{j}^{1}+2 \nabla_{2} R_{2 i 1 j}(0)+2 \nabla_{2} R_{2 j 1 i}(0) \\
& +2 \nabla_{2} R_{121 i}(0) \delta_{j}^{1}+2 \nabla_{2} R_{121 j}(0) \delta_{i}^{1}, \\
\ddot{R}_{i j}^{\prime \prime}(0,0)=-[ & 2 R_{2 i 2 j}(0)-2 R_{1 i 1 j}(0)+\delta_{i}^{2} R_{121 j}(0)+\delta_{j}^{2} R_{121 i}(0) \\
& \left.\quad+2 \delta_{i}^{1} R_{122 j}(0)+2 \delta_{j}^{1} R_{122 i}(0)+2 R_{1212}(0) \delta_{i}^{1} \delta_{j}^{1}\right] \\
+ & 2 \nabla_{1} \nabla_{1} R_{2 i 2 j}(0)-4 \nabla_{1} \nabla_{1} R_{1 i 1 j}(0)+\delta_{i}^{2} \nabla_{1} \nabla_{1} R_{121 j}(0) \\
& \quad+\delta_{j}^{2} \nabla_{1} \nabla_{1} R_{121 i}(0)+2 \delta_{i}^{1} \nabla_{1} \nabla_{1} R_{122 j}(0) \\
& \left.\quad+2 \delta_{j}^{1} \nabla_{1} \nabla_{1} R_{122 i}(0)+2 \nabla_{1} \nabla_{1} R_{1212}(0) \delta_{i}^{1} \delta_{j}^{1}\right] \\
+ & 2\left[2 \nabla_{1} \nabla_{2} R_{2 i 1 j}(0)+2 \nabla_{1} \nabla_{2} R_{2 j 1 i}(0)+2 \nabla_{1} \nabla_{2} R_{121 i}(0) \delta_{j}^{1}\right. \\
& \left.+2 \nabla_{1} \nabla_{2} R_{121 j}(0) \delta_{i}^{1}\right]+\mathcal{B}(\varepsilon) \\
=\mathcal{B}(\varepsilon) &
\end{aligned}
$$

Proof. - As we see $R_{i j}(\theta, t)=0$ when $i=1$ or $j=1$, we study the cases $i, j>1$.

Recall $e_{i}(\theta, t)=Y_{i}^{j}(\theta, t) \partial_{j} \forall i$, in particular $e_{1}(\theta, t)=\dot{X}^{j}(\theta, t) \partial_{j}$. By $(2.6)$, we can write

$$
\begin{aligned}
R_{i j}(\theta, t) & =\left\langle R\left(e_{i}(\theta, t), e_{1}(\theta, t)\right) e_{1}(\theta, t), e_{j}(\theta, t)\right\rangle \\
& =R_{d c b a} \dot{X}^{a} Y_{i}^{b} \dot{X}^{c} Y_{j}^{d} \\
& =R_{a b c d} \dot{X}^{a} Y_{i}^{b} \dot{X}^{c} Y_{j}^{d} .
\end{aligned}
$$

Here the last equality follows from the symmetry of the Riemann curvature tensor.

(1). - Differentiating (B.5) with respect to $t$, we deduce

$$
\begin{aligned}
\dot{R}_{i j}(\theta, t)= & \partial_{p} R_{a b c d} \dot{X}^{a} Y_{i}^{b} \dot{X}^{c} Y_{j}^{d} \dot{X}^{p}+R_{a b c d} \partial_{t}\left(\dot{X}^{a} Y_{i}^{b} \dot{X}^{c} Y_{j}^{d}\right) \\
= & \partial_{p} R_{a b c d} \dot{X}^{a} Y_{i}^{b} \dot{X}^{c} Y_{j}^{d} \dot{X}^{p} \\
& +R_{a b c d}\left(\ddot{X}^{a} Y_{i}^{b} \dot{X}^{c} Y_{j}^{d}+\dot{X}^{a} \dot{Y}_{i}^{b} \dot{X}^{c} Y_{j}^{d}+\dot{X}^{a} Y_{i}^{b} \ddot{X}^{c} Y_{j}^{d}+\dot{X}^{a} Y_{i}^{b} \dot{X}^{c} \dot{Y}_{j}^{d}\right) .
\end{aligned}
$$

Using the relations $X(0, t)=(t, 0, \ldots, 0)^{T}, Y(0, t)=I_{n}$, we infer on the axis

$$
\dot{R}_{i j}(0, t)=\partial_{1} R_{1 i 1 j}(X(0, t)) .
$$


Recall the first covariant derivative formula

$$
\nabla_{p} R_{a b c d}=\partial_{p} R_{a b c d}-\Gamma_{p a}^{h} R_{h b c d}-\Gamma_{p b}^{h} R_{a h c d}-\Gamma_{p c}^{h} R_{a b h d}-\Gamma_{p d}^{h} R_{a b c h} .
$$

Since the Christoffel symbols vanish identically on the axis, we have

$$
\dot{R}_{i j}(0, t)=\nabla_{1} R_{1 i 1 j} .
$$

Differentiating (B.5) with respect to $\theta$, we obtain

$$
\begin{aligned}
& R_{i j}^{\prime}(\theta, t) \\
& =\partial_{p} R_{a b c d} \dot{X}^{a} Y_{i}^{b} \dot{X}^{c} Y_{j}^{d} X^{\prime p}+R_{a b c d} \partial_{\theta}\left(\dot{X}^{a} Y_{i}^{b} \dot{X}^{c} Y_{j}^{d}\right) \\
& =\partial_{p} R_{a b c d} \dot{X}^{a} Y_{i}^{b} \dot{X}^{c} Y_{j}^{d} X^{\prime p} \\
& \quad+R_{a b c d}\left(\dot{X}^{\prime a} Y_{i}^{b} \dot{X}^{c} Y_{j}^{d}+\dot{X}^{a} Y_{i}^{\prime b} \dot{X}^{c} Y_{j}^{d}+\dot{X}^{a} Y_{i}^{b} \dot{X}^{\prime c} Y_{j}^{d}+\dot{X}^{a} Y_{i}^{b} \dot{X}^{c} Y_{j}^{\prime d}\right) .
\end{aligned}
$$

Using the relations $X(0, t)=(t, 0, \ldots, 0)^{T}, X^{\prime 1}(0, t)=0, Y(0, t)=I_{n}$, we infer

$$
\begin{aligned}
R_{i j}^{\prime}(0, t) & =\partial_{\alpha} R_{1 i 1 j} X^{\alpha^{\prime}}+R_{\alpha i 1 j} \dot{X}^{\alpha^{\prime}}+R_{1 \alpha 1 j} Y_{i}^{\alpha^{\prime}}+R_{1 i \alpha j} \dot{X}^{\alpha^{\prime}}+R_{1 i 1 \alpha} Y_{j}^{\alpha^{\prime}} \\
& =\nabla_{\alpha} R_{1 i 1 j} X^{\alpha^{\prime}}+\left(R_{\alpha i 1 j}+R_{1 i \alpha j}\right) \dot{X}^{\alpha^{\prime}}+R_{1 \alpha 1 j} Y_{i}^{\alpha^{\prime}}+R_{1 i 1 \alpha} Y_{j}^{\alpha^{\prime}}
\end{aligned}
$$

since the Christoffel symbols vanish identically on the axis. From Lemma B.1 and Lemma B.2, we get

$$
\begin{aligned}
R_{i j}^{\prime}(0, t) & =\nabla_{\alpha} R_{1 i 1 j} X^{\prime \alpha}+\left(R_{\alpha i 1 j}+R_{1 i \alpha j}\right) \dot{X}^{\prime \alpha}+\mathcal{B}\left(\varepsilon t^{2}\right) \\
& =\nabla_{2} R_{1 i 1 j}{X^{\prime}}^{2}+\left(R_{2 i 1 j}+R_{1 i 2 j}\right) \dot{X}^{\prime 2}+\mathcal{B}\left(\varepsilon t^{2}\right) \\
& =\nabla_{2} R_{1 i 1 j} \sin t+\left(R_{1 i 2 j}+R_{1 j 2 i}\right) \cos t+\mathcal{B}\left(\varepsilon t^{2}\right) .
\end{aligned}
$$

(2). - Differentiating (B.6) with respect to $t$ and using the relation $X(0, t)=(t, 0, \ldots, 0)^{T}$, we get

$$
\ddot{R}_{i j}(0, t)=\partial_{1} \nabla_{1} R_{1 i 1 j}(X(0, t))=\nabla_{11}^{2} R_{1 i 1 j}(X(0, t)),
$$

since the Christoffel symbols vanish identically on the axis. Differentiating (B.5) with respect to $\theta$ and $t$ respectively, there holds

$$
\begin{aligned}
\dot{R}_{i j}^{\prime}(\theta, t) & \partial_{p q}^{2} R_{a b c d} \dot{X}^{a} Y_{i}^{b} \dot{X}^{c} Y_{j}^{d} \dot{X}^{q} X^{\prime p}+\partial_{p} R_{a b c d} \dot{X}^{a} Y_{i}^{b} \dot{X}^{c} Y_{j}^{d} \dot{X}^{\prime p} \\
& +\partial_{p} R_{a b c d} \partial_{t}\left(\dot{X}^{a} Y_{i}^{b} \dot{X}^{c} Y_{j}^{d}\right) X^{\prime p}+\partial_{p} R_{a b c d} \partial_{\theta}\left(\dot{X}^{a} Y_{i}^{b} \dot{X}^{c} Y_{j}^{d}\right) \dot{X}^{p} \\
& +R_{a b c d} \partial_{t \theta}^{2}\left(\dot{X}^{a} Y_{i}^{b} \dot{X}^{c} Y_{j}^{d}\right)
\end{aligned}
$$




$$
\begin{aligned}
= & \partial_{p q}^{2} R_{a b c d} \dot{X}^{a} Y_{i}^{b} \dot{X}^{c} Y_{j}^{d} \dot{X}^{q} X^{\prime p}+\partial_{p} R_{a b c d} \dot{X}^{a} Y_{i}^{b} \dot{X}^{c} Y_{j}^{d} \dot{X}^{\prime p} \\
& +\partial_{p} R_{a b c d}\left(\ddot{X}^{a} Y_{i}^{b} \dot{X}^{c} Y_{j}^{d}+\dot{X}^{a} \dot{Y}_{i}^{b} \dot{X}^{c} Y_{j}^{d}+\dot{X}^{a} Y_{i}^{b} \ddot{X}^{c} Y_{j}^{d}+\dot{X}^{a} Y_{i}^{b} \dot{X}^{c} \dot{Y}_{j}^{d}\right) X^{\prime p} \\
& +\partial_{p} R_{a b c d}\left(\dot{X}^{\prime a} Y_{i}^{b} \dot{X}^{c} Y_{j}^{d}+\dot{X}^{a} Y^{\prime b}{ }_{i} \dot{X}^{c} Y_{j}^{d}+\dot{X}^{a} Y_{i}^{b} \dot{X}^{\prime c} Y_{j}^{d}+\dot{X}^{a} Y_{i}^{b} \dot{X}^{c} Y^{\prime d}{ }_{j}^{d}\right) \dot{X}^{p} \\
& +R_{a b c d}\left(\ddot{X}^{\prime}{ }^{a} Y_{i}^{b} \dot{X}^{c} Y_{j}^{d}+\dot{X}^{\prime a} \dot{Y}_{i}^{b} \dot{X}^{c} Y_{j}^{d}+\dot{X}^{\prime a} Y_{i}^{b} \ddot{X}^{c} Y_{j}^{d}+\dot{X}^{\prime a} Y_{i}^{b} \dot{X}^{c} \dot{Y}_{j}^{d}\right. \\
& +\ddot{X}^{a} Y^{\prime}{ }_{i} \dot{X}^{c} Y_{j}^{d}+\dot{X}^{a} \dot{Y}^{\prime}{ }_{i}^{b} \dot{X}^{c} Y_{j}^{d}+\dot{X}^{a} Y^{\prime}{ }_{i} \ddot{X}^{c} Y_{j}^{d}+\dot{X}^{a} Y^{\prime b}{ }_{i} \dot{X}^{c} \dot{Y}_{j}^{d} \\
& +\ddot{X}^{a} Y_{i}^{b} \dot{X}^{\prime c} Y_{j}^{d}+\dot{X}^{a} \dot{Y}_{i}^{b} \dot{X}^{\prime c} Y_{j}^{d}+\dot{X}^{a} \dot{Y}_{i}^{b} \ddot{X}^{\prime}{ }^{c} Y_{j}^{d}+\dot{X}^{a} Y_{i}^{b} \dot{X}^{\prime c} \dot{Y}_{j}^{d} \\
& \left.+\ddot{X}^{a} Y_{i}^{b} \dot{X}^{c} Y^{\prime d}{ }_{j}^{d}+\dot{X}^{a} \dot{Y}_{i}^{b} \dot{X}^{c} Y^{\prime}{ }_{j}^{d}+\dot{X}^{a} Y_{i}^{b} \ddot{X}^{c} Y^{\prime}{ }_{j}^{d}+\dot{X}^{a} Y_{i}^{b} \dot{X}^{c} \dot{Y}^{\prime}{ }_{j}^{d}\right) .
\end{aligned}
$$

Using the relations $X(0, t)=(t, 0, \ldots, 0)^{T}, X^{\prime 1}(0, t)=0, Y(0, t)=I_{n}$, we infer on the axis

$$
\begin{aligned}
\dot{R}_{i j}^{\prime}(0, t)= & \partial_{1 \alpha}^{2} R_{1 i 1 j} X^{\prime \alpha}+\partial_{\alpha} R_{1 i 1 j} \dot{X}^{\prime \alpha}+\partial_{1} R_{\alpha i 1 j} \dot{X}^{\prime \alpha}+\partial_{1} R_{1 \alpha 1 j} Y_{i}^{\prime \alpha} \\
& +\partial_{1} R_{1 i \alpha j} \dot{X}^{\prime \alpha}+\partial_{1} R_{1 i 1 \alpha} Y_{j}^{\prime \alpha}+R_{\alpha i 1 j} \ddot{X}^{\prime \alpha} \\
& +R_{1 \alpha 1 j} \dot{Y}^{\prime \alpha}{ }_{i}^{\alpha}+R_{1 i \alpha j} \ddot{X}^{\prime \alpha}+R_{1 i 1 \alpha} \dot{Y}^{\prime \alpha}{ }_{j} \\
= & \partial_{1 \alpha}^{2} R_{1 i 1 j} X^{\prime \alpha}+\left(\partial_{\alpha} R_{1 i 1 j}+\partial_{1} R_{\alpha i 1 j}+\partial_{1} R_{1 i \alpha j}\right) \dot{X}^{\prime \alpha} \\
& +\left(R_{\alpha i 1 j}+R_{1 i \alpha j}\right) \ddot{X}^{\prime \alpha}+\partial_{1} R_{1 \alpha 1 j} Y^{\prime \alpha}{ }_{i}^{\alpha}+\partial_{1} R_{1 i 1 \alpha} Y_{j}^{\prime \alpha} \\
& +R_{1 \alpha 1 j} \dot{Y}^{\prime \alpha}{ }_{i}^{\alpha}+R_{1 i 1 \alpha} \dot{Y}^{\prime \alpha}{ }_{j} .
\end{aligned}
$$

Using the fact $\nabla_{p} R_{a b c d}=\partial_{p} R_{a b c d}$ on the axis, it follows from Lemma B.1 and Lemma B.2 that on the axis there holds

$$
\partial_{1 p}^{2} R_{a b c d}=\nabla_{1 p}^{2} R_{a b c d},
$$

and

$$
\begin{aligned}
\dot{R}_{i j}^{\prime}(0, t)= & \nabla_{1 \alpha}^{2} R_{1 i 1 j} X^{\prime \alpha}+\left(\nabla_{\alpha} R_{1 i 1 j}+\nabla_{1} R_{\alpha i 1 j}+\nabla_{1} R_{1 i \alpha j}\right) \dot{X}^{\prime \alpha} \\
& +\left(R_{\alpha i 1 j}+R_{1 i \alpha j}\right) \ddot{X}^{\prime \alpha}+\nabla_{1} R_{1 \alpha 1 j} Y_{i}^{\prime \alpha}+\nabla_{1} R_{1 i 1 \alpha} Y_{j}^{\prime \alpha} \\
& +R_{1 \alpha 1 j} \dot{Y}^{\prime \alpha}{ }_{i}+R_{1 i 1 \alpha} \dot{Y}^{\prime \alpha}{ }_{j} \\
= & \nabla_{1 \alpha}^{2} R_{1 i 1 j} X^{\prime \alpha}+\left(\nabla_{\alpha} R_{1 i 1 j}+\nabla_{1} R_{\alpha i 1 j}+\nabla_{1} R_{1 i \alpha j}\right) \dot{X}^{\prime \alpha} \\
& +\left(R_{\alpha i 1 j}+R_{1 i \alpha j}\right) \ddot{X}^{\prime \alpha}+\mathcal{B}(\varepsilon t) \\
= & \nabla_{12}^{2} R_{1 i 1 j} X^{\prime 2}+\left(\nabla_{2} R_{1 i 1 j}+\nabla_{1} R_{2 i 1 j}+\nabla_{1} R_{1 i 2 j}\right) \dot{X}^{\prime 2} \\
& +\left(R_{2 i 1 j}+R_{1 i 2 j}\right) \ddot{X}^{\prime}+\mathcal{B}(\varepsilon t) \\
= & \nabla_{12}^{2} R_{1 i 1 j} \sin t+\left(\nabla_{2} R_{1 i 1 j}+\nabla_{1} R_{2 i 1 j}+\nabla_{1} R_{1 i 2 j}\right) \cos t \\
& -\left(R_{2 i 1 j}+R_{1 i 2 j}\right) \sin t+\mathcal{B}(\varepsilon t) \\
= & \left(\nabla_{1} R_{1 i 2 j}+\nabla_{1} R_{1 j 2 i}+\nabla_{2} R_{1 i 1 j}\right) \cos t+\mathcal{B}(\varepsilon t) .
\end{aligned}
$$

Here in the last equality we used the fact that $R_{1 i 2 j}=\mathcal{B}(\varepsilon)$. 
Differentiating (B.5) twice with respect to $\theta$, we get

$$
\begin{aligned}
& R_{i j}^{\prime \prime}(\theta, t) \\
& =\partial_{p q}^{2} R_{a b c d} \dot{X}^{a} Y_{i}^{b} \dot{X}^{c} Y_{j}^{d} X^{\prime p} X^{\prime q}+\partial_{p} R_{a b c d} \dot{X}^{a} Y_{i}^{b} \dot{X}^{c} Y_{j}^{d} X^{p^{\prime \prime}} \\
& +2 \partial_{p} R_{a b c d} \partial_{\theta}\left(\dot{X}^{a} Y_{i}^{b} \dot{X}^{c} Y_{j}^{d}\right) X^{\prime p}+R_{a b c d} \partial_{\theta \theta}^{2}\left(\dot{X}^{a} Y_{i}^{b} \dot{X}^{c} Y_{j}^{d}\right) \\
& =\partial_{p q}^{2} R_{a b c d} \dot{X}^{a} Y_{i}^{b} \dot{X}^{c} Y_{j}^{d} X^{\prime p} X^{\prime q}+\partial_{p} R_{a b c d} \dot{X}^{a} Y_{i}^{b} \dot{X}^{c} Y_{j}^{d} X^{\prime \prime p} \\
& +2 \partial_{p} R_{a b c d}\left(\dot{X}^{\prime a} Y_{i}^{b} \dot{X}^{c} Y_{j}^{d}+\dot{X}^{a} Y_{i}^{\prime b} \dot{X}^{c} Y_{j}^{d}+\dot{X}^{a} Y_{i}^{b} \dot{X}^{\prime c} Y_{j}^{d}+\dot{X}^{a} Y_{i}^{b} \dot{X}^{c} Y_{j}^{\prime d}\right) X^{\prime p} \\
& +R_{a b c d}\left(\dot{X}^{\prime \prime a} Y_{i}^{b} \dot{X}^{c} Y_{j}^{d}+2 \dot{X}^{\prime a} Y_{i}^{\prime b} \dot{X}^{c} Y_{j}^{d}+2 \dot{X}^{\prime a} Y_{i}^{b} \dot{X}^{\prime c} Y_{j}^{d}+2 \dot{X}^{\prime a} Y_{i}^{b} \dot{X}^{c} Y_{j}^{\prime d}\right. \\
& +\dot{X}^{a} Y^{\prime \prime}{ }_{i}^{b} \dot{X}^{c} Y_{j}^{d}+2 \dot{X}^{a} Y_{i}^{\prime b} \dot{X}^{\prime c} Y_{j}^{d}+2 \dot{X}^{a} Y_{i}^{\prime b} \dot{X}^{c} Y_{j}^{\prime d} \\
& \left.+\dot{X}^{a} \dot{Y}_{i}^{b} \dot{X}^{\prime \prime c} Y_{j}^{d}+2 \dot{X}^{a} Y_{i}^{b} \dot{X}^{\prime c} Y_{j}^{\prime d}+\dot{X}^{a} Y_{i}^{b} \dot{X}^{c} Y_{j}^{\prime \prime}{ }_{j}^{d}\right) \text {. }
\end{aligned}
$$

Applying the relations $X(0, t)=(t, 0, \ldots, 0)^{T}, X^{\prime 1}(0, t)=0, Y(0, t)=I_{n}$, we deduce on the axis

$$
\begin{aligned}
& R_{i j}^{\prime \prime}(0, t)=\partial_{\alpha \beta}^{2} R_{1 i 1 j} X^{\prime \alpha} X^{\prime \beta}+\partial_{p} R_{1 i 1 j} X^{\prime \prime p}+2 \partial_{\alpha} R_{\beta i 1 j} X^{\prime \alpha} \dot{X}^{\prime \beta} \\
& +2 \partial_{\alpha} R_{1 \beta 1 j} X^{\prime \alpha} Y_{i}^{\prime \beta}+2 \partial_{\alpha} R_{1 i \beta j} X^{\prime \alpha} \dot{X}^{\prime \beta}+2 \partial_{\alpha} R_{1 i 1 \beta} X^{\prime \alpha} Y_{j}^{\prime \beta} \\
& +R_{a i 1 j} \dot{X}^{\prime \prime a}+2 R_{\alpha b 1 j} \dot{X}^{\prime \alpha} Y_{i}^{\prime b}+2 R_{\alpha i \beta j} \dot{X}^{\prime \alpha} \dot{X}^{\prime \beta}+2 R_{\alpha i 1 \beta} \dot{X}^{\prime \alpha} Y_{j}^{\prime \beta} \\
& +R_{1 \beta 1 j} Y_{i}^{\prime \prime \beta}+2 R_{1 \beta \alpha j} \dot{X}^{\prime \alpha} Y_{i}^{\prime \beta}+2 R_{1 \alpha 1 \beta} Y_{i}^{\prime \alpha} Y_{j}^{\prime \beta}+R_{1 i a j} \dot{X}^{\prime \prime a} \\
& +2 R_{1 i \alpha b} \dot{X}^{\prime \alpha} Y_{j}^{\prime b}+R_{1 i 1 \beta} Y_{j}^{\prime \prime \beta} \\
& =\partial_{\alpha \beta}^{2} R_{1 i 1 j} X^{\prime \alpha} X^{\prime \beta}+\partial_{p} R_{1 i 1 j} X^{\prime \prime p}+2\left(\partial_{\alpha} R_{\beta i 1 j}+\partial_{\alpha} R_{1 i \beta j}\right) X^{\prime \alpha} \dot{X}^{\prime \beta} \\
& +2\left(\partial_{\alpha} R_{1 \beta 1 j} X^{\prime \alpha} Y_{i}^{\prime \beta}+\partial_{\alpha} R_{1 i 1 \beta} X^{\prime \alpha} Y_{j}^{\prime \beta}\right)+\left(R_{a i 1 j}+R_{1 i a j}\right) \dot{X}^{\prime \prime a} \\
& +2 R_{\alpha i \beta j} \dot{X}^{\prime \alpha} \dot{X}^{\prime \beta}+2\left(R_{\alpha b 1 j} \dot{X}^{\prime \alpha} Y_{i}^{\prime b}+R_{1 i \alpha b} \dot{X}^{\prime \alpha} Y_{j}^{\prime b}\right) \\
& +2\left(R_{\alpha i 1 \beta} \dot{X}^{\prime \alpha} Y_{j}^{\prime \beta}+R_{1 \beta \alpha j} \dot{X}^{\prime \alpha} Y_{i}^{\prime \beta}\right) \\
& +2 R_{1 \alpha 1 \beta} Y_{i}^{\prime \alpha} Y_{j}^{\prime \beta}+R_{1 \beta 1 j} Y_{i}^{\prime \prime \beta}+R_{1 i 1 \beta} Y_{j}^{\prime \prime \beta} \text {. }
\end{aligned}
$$

It is clear that on the axis $\nabla_{p} R_{a b c d}=\partial_{p} R_{a b c d}$. On the other hand, using Lemma 2.5, we deduce on the axis

$$
\begin{aligned}
\partial_{\alpha \beta}^{2} R_{1 i 1 j}= & \nabla_{\alpha \beta}^{2} R_{1 i 1 j}+\partial_{\alpha} \Gamma_{1 \beta}^{p} R_{p i 1 j}+\partial_{\alpha} \Gamma_{i \beta}^{p} R_{1 p 1 j} \\
& \quad+\partial_{\alpha} \Gamma_{1 \beta}^{p} R_{1 i p j}+\partial_{\alpha} \Gamma_{j \beta}^{p} R_{1 i 1 p} \\
= & \nabla_{\alpha \beta}^{2} R_{1 i 1 j}+\partial_{\alpha} \Gamma_{1 \beta}^{p}\left(R_{p i 1 j}+R_{1 i p j}\right) \\
& \quad+\partial_{\alpha} \Gamma_{i \beta}^{p} R_{1 p 1 j}+\partial_{\alpha} \Gamma_{j \beta}^{p} R_{1 i 1 p} \\
= & \nabla_{\alpha \beta}^{2} R_{1 i 1 j}+R_{\beta \alpha 1}^{p}\left(R_{1 i p j}+R_{1 j p i}\right) \\
& \quad+\frac{1}{3}\left(R_{i \alpha \beta}^{p}+R_{\beta \alpha i}^{p}\right) R_{1 p 1 j}+\frac{1}{3}\left(R_{j \alpha \beta}^{p}+R_{\beta \alpha j}^{p}\right) R_{1 i 1 p} .
\end{aligned}
$$


Thus $\partial_{\alpha \beta}^{2} R_{1 i 1 j}$ are uniformly bounded on the axis. Therefore

$$
\begin{aligned}
R_{i j}^{\prime \prime}(0, t)= & \partial_{\alpha \beta}^{2} R_{1 i 1 j} X^{\prime \alpha} X^{\prime \beta}+\nabla_{p} R_{1 i 1 j} X^{\prime \prime p}+2\left(\nabla_{\alpha} R_{\beta i 1 j}+\nabla_{\alpha} R_{1 i \beta j}\right) X^{\prime \alpha} \dot{X}^{\prime \beta} \\
& +2\left(\nabla_{\alpha} R_{1 \beta 1 j} X^{\prime \alpha} Y_{i}^{\prime \beta}+\nabla_{\alpha} R_{1 i 1 \beta} X^{\prime \alpha} Y_{j}^{\prime \beta}\right)+\left(R_{a i 1 j}+R_{1 i a j}\right) \dot{X}^{\prime \prime}{ }^{a} \\
& +2 R_{\alpha i \beta j} \dot{X}^{\prime \alpha} \dot{X}^{\prime \beta}+2\left(R_{\alpha b 1 j} \dot{X}^{\prime \alpha} Y_{i}^{\prime b}+R_{1 i \alpha b} \dot{X}^{\prime \alpha} Y_{j}^{\prime b}\right) \\
& +2\left(R_{\alpha i 1 \beta} \dot{X}^{\prime \alpha} Y_{j}^{\prime \beta}+R_{1 \beta \alpha j} \dot{X}^{\prime \alpha} Y_{i}^{\prime \beta}\right)+2 R_{1 \alpha 1 \beta} Y_{i}^{\prime \alpha} Y_{j}^{\prime \beta} \\
& +R_{1 \beta 1 j} Y_{i}^{\prime \prime}{ }_{i}^{\beta}+R_{1 i 1 \beta} Y_{j}^{\prime \prime}{ }_{j} .
\end{aligned}
$$

With the help of Lemma 2.5 and Lemma B.1, we obtain

$$
\begin{aligned}
R_{i j}^{\prime \prime}(0, t)= & \partial_{22}^{2} R_{1 i 1 j} X^{\prime 2} X^{\prime 2}+\nabla_{1} R_{1 i 1 j} X^{\prime \prime}+2\left(\nabla_{2} R_{2 i 1 j}+\nabla_{2} R_{1 i 2 j}\right) X^{\prime 2} \dot{X}^{\prime 2} \\
& +2 R_{1 i 1 j} \dot{X}^{\prime \prime 1}+2 R_{2 i 2 j} \dot{X}^{\prime 2} \dot{X}^{\prime 2} \\
& +2\left(\delta_{2 i} R_{211 j} \dot{X}^{\prime 2} Y_{2}^{\prime 1}+R_{1 i 21} \delta_{2 j} \dot{X}^{\prime 2} Y^{\prime 1}{ }_{2}\right) \\
& +R_{1 i 1 j}{Y^{\prime \prime}}^{i}+R_{1 i 1 j} Y^{\prime \prime}{ }_{j}+\mathcal{B}\left(\varepsilon t^{2}\right) \\
= & \partial_{22}^{2} R_{1 i 1 j} \sin ^{2} t-\nabla_{1} R_{1 i 1 j} \sin t \cos t \\
& +2\left(\nabla_{2} R_{2 i 1 j}+\nabla_{2} R_{1 i 2 j}\right) \sin t \cos t-2 R_{1 i 1 j} \cos (2 t)+2 R_{2 i 2 j} \cos ^{2} t \\
& +2\left(\delta_{2 i}+\delta_{2 j}\right) R_{1 i 1 j} \cos ^{2} t-\left(\delta_{2 i}+\delta_{2 j}\right) R_{1 i 1 j} \cos ^{2} t \\
& +\frac{1}{3}\left(\delta_{i 3}+\delta_{j 3}+\cdots+\delta_{i n}+\delta_{j n}\right) R_{1 i 1 j} \sin ^{2} t+\mathcal{B}\left(\varepsilon t^{2}\right) \\
= & \partial_{22}^{2} R_{1 i 1 j} \sin ^{2} t+\left(-\nabla_{1} R_{1 i 1 j}+2 \nabla_{2} R_{2 i 1 j}+2 \nabla_{2} R_{1 i 2 j}\right) \sin t \cos t \\
& -2 R_{1 i 1 j} \cos (2 t)+2 R_{2 i 2 j} \cos ^{2} t+\left(\delta_{2 i}+\delta_{2 j}\right) R_{1 i 1 j} \cos ^{2} t \\
& +\frac{1}{3}\left(\delta_{i 3}+\delta_{j 3}+\cdots+\delta_{i n}+\delta_{j n}\right) R_{1 i 1 j} \sin ^{2} t+\mathcal{B}\left(\varepsilon t^{2}\right) .
\end{aligned}
$$

In view of (2.17) and by the formula for the second covariant derivative, we have on the axis

$$
\begin{aligned}
& \partial_{22}^{2} R_{1 i 1 j} \\
& =\nabla_{22}^{2} R_{1 i 1 j}+\partial_{2} \Gamma_{21}^{p} R_{p i 1 j}+\partial_{2} \Gamma_{2 i}^{p} R_{1 p 1 j}+\partial_{2} \Gamma_{21}^{p} R_{1 i p j}+\partial_{2} \Gamma_{2 j}^{p} R_{1 i 1 p} \\
& =\nabla_{22}^{2} R_{1 i 1 j}+R_{221}^{p}\left(R_{p i 1 j}+R_{1 i p j}\right)+\frac{1}{3} R_{22 i}^{p} R_{1 p 1 j}+\frac{1}{3} R_{22 j}^{p} R_{1 i 1 p} \\
& =-\frac{8}{3} R_{1 i 1 j}+\frac{1}{3}\left(\delta_{2 i}+\delta_{2 j}\right) R_{1 i 1 j}+\mathcal{B}(\varepsilon) .
\end{aligned}
$$


Therefore, we infer on the axis

$$
\begin{aligned}
R_{i j}^{\prime \prime}(0, t)= & -\frac{8}{3} R_{1 i 1 j} \sin ^{2} t+\frac{1}{3}\left(\delta_{2 i}+\delta_{2 j}\right) R_{1 i 1 j} \sin ^{2} t \\
& +\left(-\nabla_{1} R_{1 i 1 j}+2 \nabla_{2} R_{2 i 1 j}+2 \nabla_{2} R_{1 i 2 j}\right) \sin t \cos t \\
& -2 R_{1 i 1 j} \cos (2 t)+2 R_{2 i 2 j} \cos ^{2} t+\left(\delta_{2 i}+\delta_{2 j}\right) R_{1 i 1 j} \cos ^{2} t \\
& +\frac{1}{3}\left(\delta_{i 3}+\delta_{j 3}+\cdots+\delta_{i n}+\delta_{j n}\right) R_{1 i 1 j} \sin ^{2} t+\mathcal{B}\left(\varepsilon t^{2}\right) .
\end{aligned}
$$

Since for $i, j>1, \delta_{i 3}+\cdots+\delta_{i n}=1-\delta_{2 i}, \delta_{j 3}+\cdots+\delta_{j n}=1-\delta_{2 j}$ so that there holds on the axis

$$
\begin{aligned}
R_{i j}^{\prime \prime}(0, t)= & -\frac{8}{3} R_{1 i 1 j} \sin ^{2} t+\frac{1}{3}\left(\delta_{2 i}+\delta_{2 j}\right) R_{1 i 1 j} \sin ^{2} t \\
& +\left(-\nabla_{1} R_{1 i 1 j}+2 \nabla_{2} R_{2 i 1 j}+2 \nabla_{2} R_{1 i 2 j}\right) \sin t \cos t \\
& -2 R_{1 i 1 j} \cos (2 t)+2 R_{2 i 2 j} \cos ^{2} t+\left(\delta_{2 i}+\delta_{2 j}\right) R_{1 i 1 j} \cos ^{2} t \\
& +\frac{1}{3}\left(2-\delta_{2 i}-\delta_{2 j}\right) R_{1 i 1 j} \sin ^{2} t+\mathcal{B}\left(\varepsilon t^{2}\right) \\
= & -2 R_{1 i 1 j} \sin ^{2} t+\left(-\nabla_{1} R_{1 i 1 j}+2 \nabla_{2} R_{2 i 1 j}+2 \nabla_{2} R_{1 i 2 j}\right) \sin t \cos t \\
& -2 R_{1 i 1 j} \cos (2 t)+2 R_{2 i 2 j} \cos ^{2} t+\left(\delta_{2 i}+\delta_{2 j}\right) R_{1 i 1 j} \cos ^{2} t+\mathcal{B}\left(\varepsilon t^{2}\right) \\
= & 2\left(R_{2 i 2 j}-R_{1 i 1 j}\right) \cos ^{2} t \\
& +\left(-\nabla_{1} R_{1 i 1 j}+2 \nabla_{2} R_{1 i 2 j}+2 \nabla_{2} R_{1 j 2 i}\right) \sin t \cos t \\
& +\left(\delta_{2 i}+\delta_{2 j}\right) R_{1 i 1 j} \cos ^{2} t+\mathcal{B}\left(\varepsilon t^{2}\right) .
\end{aligned}
$$

(3). - - It is a direct consequence of the second part. This ends the proof of the Proposition B.3.

As a direct consequence of Proposition B.3, we have the following result.

COROllary B.4. - Under the curvature assumptions (1.3) and (1.4) (or (1.5)) with $\varepsilon<1$, then in the Fermi chart, we have on the axis: for all $t \in[0, \tau]$ with $\tau=\left|\nu_{0}\right|$
(1) $\dot{R}^{\prime}(0,0)=\left[\begin{array}{cc}0 & 0 \\ 0 & \nabla_{1} R_{1 i 2 j}+\nabla_{1} R_{1 j 2 i}+\nabla_{2} R_{1 i 1 j}\end{array}\right]$;
(2) $R^{\prime}(0, t)=\left[\begin{array}{cc}0 & 0 \\ 0 & \mathcal{B}(\varepsilon)\end{array}\right], \dot{R}^{\prime}(0, t)=\left[\begin{array}{cc}0 & 0 \\ 0 & \mathcal{B}(\varepsilon)\end{array}\right], R^{\prime \prime}(0, t)=\left[\begin{array}{cc}0 & 0 \\ 0 & \mathcal{B}(\varepsilon)\end{array}\right]$.

\section{B.1.4. Asymptotic behaviour near the origin}

In this part, we deduce the asymptotic behavior of the $\mathcal{C}$-curvature near the origin. We first take account of the asymptotic behavior of the coefficient $a_{11}$ which involves $\ddot{S}$. 
Lemma B.5. - Under the curvature assumptions (1.3) and (1.4) (or (1.5)) with $\varepsilon<1$, we have on the axis for small $t \geqslant 0$

$$
\begin{aligned}
S(0, t) & =I_{n}-\frac{t^{2}}{3} R(0,0)-\frac{t^{3}}{12} \dot{R}(0 ; 0)-\frac{t^{4}}{45} A+O\left(\varepsilon t^{4}+t^{6}\right), \\
\dot{S}(0, t) & =-\frac{2}{3} t R(0,0)-\frac{t^{2}}{4} \dot{R}(0,0)-\frac{4}{45} t^{3} A+O\left(\varepsilon t^{3}+t^{5}\right), \\
\ddot{S}(0, t) & =-\frac{2}{3} R(0,0)-\frac{t}{2} \dot{R}(0,0)-\frac{4}{15} t^{2} A+O\left(\varepsilon t^{2}+t^{4}\right), \\
S^{\prime}(0, t) & =-\frac{t^{2}}{3} R^{\prime}(0,0)-\frac{t^{3}}{12} \dot{R}^{\prime}(0,0)+O\left(\varepsilon t^{4}+t^{6}\right), \\
\dot{S}^{\prime}(0, t) & =-\frac{2}{3} t R^{\prime}(0,0)-\frac{t^{2}}{4} \dot{R}^{\prime}(0,0)+O\left(\varepsilon t^{3}+t^{5}\right), \\
S^{\prime \prime}(0, t) & =-\frac{t^{2}}{3} R^{\prime \prime}(0,0)-\frac{t^{3}}{12} \dot{R}^{\prime \prime}(0,0)+O\left(\varepsilon t^{4}+t^{6}\right) .
\end{aligned}
$$

where $A=\left[\begin{array}{ll}0 & 0 \\ 0 & I_{n-1}\end{array}\right]$ and $R$ is given by (2.6) (see also Section B.1.3)

Remark B.6. - The formula (B.9) can also recover the expression of the $\mathcal{C}$-curvature in the special case $\nu_{0}=0$. Using a Riemannian normal coordinate system at $m_{0}$, we get from (1.1),

$$
\begin{aligned}
\mathcal{C}\left(m_{0}, 0\right)(\xi, \eta) & =-\left.\frac{3}{2} \frac{\mathrm{d}^{2}}{\mathrm{~d} s^{2}}\right|_{s=0}\left\langle S\left(m_{0}, \eta, s\right)(\xi), \xi\right\rangle \\
& =\langle R(0,0) \xi, \xi\rangle=R_{m_{0}}(\xi, \eta, \xi, \eta) .
\end{aligned}
$$

Proof. - Let $\widetilde{J}_{a}(t)$ be the solution of the following second order equation

$$
\ddot{\tilde{J}}_{a}(t)+R(0,0) \widetilde{J}_{a}(t)=0, \quad \forall a=0,1
$$

with the initial conditions

$$
\begin{array}{ll}
\widetilde{J}_{0}(0)=0, & \dot{\tilde{J}}_{0}(0)=I_{n}, \\
\widetilde{J}_{1}(0)=I_{n}, & \dot{\tilde{J}}_{1}(0)=0 .
\end{array}
$$

From the representation formula (2.9), we derive

$$
\begin{aligned}
J_{0}(t)=\widetilde{J}_{0}(t)+\widetilde{J}_{0}(t) \int_{0}^{t} \widetilde{J}_{1}^{*}(s)[R(0,0)-R(0, s)] J_{0}(s) \mathrm{d} s & \\
& \quad-\widetilde{J}_{1}(t) \int_{0}^{t} \widetilde{J}_{0}^{*}[R(0,0)-R(0, s)] J_{0} \mathrm{~d} s .
\end{aligned}
$$

It follows from the Taylor formula and Proposition B.3,

$$
R(0, t)=R(0,0)+t \dot{R}(0,0)+O\left(\varepsilon t^{2}\right) .
$$


On the other hand, we have $\widetilde{J}_{0}(t)=t I_{n}-\frac{t^{3}}{6} R(0,0)+\frac{t^{5}}{120}(R(0,0))^{2}+O\left(t^{7}\right)$ so that

$$
\begin{aligned}
J_{0}(0, t) & =\widetilde{J}_{0}(t)-\frac{t^{4}}{12} \dot{R}(0,0)+O\left(\varepsilon t^{5}\right) \\
& =t I_{n}-\frac{t^{3}}{6} R(0,0)-\frac{t^{4}}{12} \dot{R}(0,0)+\frac{t^{5}}{120}(R(0,0))^{2}+O\left(\varepsilon t^{5}+t^{7}\right) \\
& =t I_{n}-\frac{t^{3}}{6} R(0,0)-\frac{t^{4}}{12} \dot{R}(0,0)+\frac{t^{5}}{120} A+O\left(\varepsilon t^{5}+t^{7}\right) .
\end{aligned}
$$

Here we use the fact $(R(0,0))^{2}=A+O(\varepsilon)$. Similarly, we have

$$
J_{1}(0, t)=I_{n}-\frac{t^{2}}{2} R(0,0)-\frac{t^{3}}{6} \dot{R}(0,0)+\frac{t^{4}}{24} A+O\left(\varepsilon t^{4}+t^{6}\right) .
$$

Gathering the above estimates, we infer

$$
\begin{aligned}
S(0, t)= & t J_{0}(0, t)^{-1} J_{1}(0, t) \\
= & t\left[t I_{n}-\frac{t^{3}}{6} R(0,0)-\frac{t^{4}}{12} \dot{R}(0,0)+\frac{t^{5}}{120} A+O\left(\varepsilon t^{5}+t^{7}\right)\right] \\
& \quad \times\left[I_{n}-\frac{t^{2}}{2} R(0,0)-\frac{t^{3}}{6} \dot{R}(0,0)+\frac{t^{4}}{24} A+O\left(\varepsilon t^{4}+t^{6}\right)\right] \\
= & \quad\left[I_{n}+\frac{t^{2}}{6} R(0,0)+\frac{t^{3}}{12} \dot{R}(0,0)+\frac{7}{360} t^{4} A+O\left(\varepsilon t^{4}+t^{6}\right)\right] \\
& \quad \quad\left[I_{n}-\frac{t^{2}}{2} R(0,0)-\frac{t^{3}}{6} \dot{R}(0,0)+\frac{t^{4}}{24} A+O\left(\varepsilon t^{4}+t^{6}\right)\right] \\
= & I_{n}-\frac{t^{2}}{3} R(0,0)-\frac{t^{3}}{12} \dot{R}(0,0)-\frac{t^{4}}{45} A+O\left(\varepsilon t^{4}+t^{6}\right) .
\end{aligned}
$$

As the term $\ddot{S}$ involves $J_{0}^{-1} \dot{J}_{0}$ and $J_{0}^{-1} \dot{J}_{1}$, we cosider the expansion of $J_{0}^{-1} \dot{J}_{0}$ and $J_{0}^{-1} \dot{J}_{1}$. Differentiating $(2.5)$ with respect to $t, \dot{J}_{0}(0, t)$ satisfies

$$
\left\{\begin{array}{l}
\ddot{J}_{0}+R \dot{J}_{0}=-\dot{R} J_{0}, \\
\dot{J}_{0}(0)=I_{n}, \quad \ddot{J}_{0}(0)=0 .
\end{array}\right.
$$

With the help of the representation formula (2.9) again, we obtain

$$
\dot{J}_{0}(0, t)=J_{1}(0, t)-J_{0}(0, t) \int_{0}^{t} J_{1}^{*} \dot{R} J_{0} \mathrm{~d} s+J_{1}(0, t) \int_{0}^{t} J_{0}^{*} \dot{R} J_{0} \mathrm{~d} s,
$$

which implies

$$
t J_{0}^{-1} \dot{J}_{0}(0, t)=t J_{0}^{-1} J_{1}(0, t)-t \int_{0}^{t} J_{1}^{*} \dot{R} J_{0} \mathrm{~d} s+t J_{0}^{-1} J_{1}(0, t) \int_{0}^{t} J_{0}^{*} \dot{R} J_{0} \mathrm{~d} s .
$$


It follows from Proposition B.3

$$
\begin{aligned}
R(0, t) & =\dot{R}(0,0)+\int_{0}^{t} \ddot{R}(0, s) \mathrm{d} s \\
& =\dot{R}(0,0)+\int_{0}^{t} \nabla_{11}^{2} R(0, s) \mathrm{d} s \\
& =\dot{R}(0,0)+O(\varepsilon t) .
\end{aligned}
$$

Hence, we deduce

$$
t J_{0}^{-1} \dot{J}_{0}(0, t)=I_{n}-\frac{t^{2}}{3} R(0,0)-\frac{t^{3}}{4} \dot{R}(0,0)-\frac{t^{4}}{45} A+O\left(\varepsilon t^{4}+t^{6}\right) .
$$

Similarly, we infer

$$
J_{0}^{-1} \dot{J}_{1}(0, t)=-R(0,0)-\frac{t}{2} \dot{R}(0,0)+O\left(\varepsilon t^{2}+t^{4}\right) .
$$

Recall the first and second derivatives of $S$ with respect to $t$

$$
\begin{aligned}
& \dot{S}=\left(I_{n}-t J_{0}^{-1} \dot{J}_{0}\right) J_{0}^{-1} J_{1}+t J_{0}^{-1} \dot{J}_{1}, \\
& \ddot{S}=2 J_{0}^{-1} \dot{J}_{1}-2 t J_{0}^{-1} \dot{J}_{0} J_{0}^{-1} \dot{J}_{1}+2 J_{0}^{-1} \dot{J}_{0}\left(t J_{0}^{-1} \dot{J}_{0}-I_{n}\right) J_{0}^{-1} J_{1} .
\end{aligned}
$$

Together with (B.15) and (B.16), we deduce (B.10) and (B.11).

We consider $J_{0}^{-1} J_{0}^{\prime}, J_{0}^{-1} J_{1}^{\prime}$. $J_{a}^{\prime}(0, t)$ satisfies the following equations

$$
\left\{\begin{array}{l}
\ddot{J}_{a}^{\prime}+R J_{a}^{\prime}=-R^{\prime} J_{a}, \quad \forall a=0,1, \\
J_{a}^{\prime}(0,0)=0=\dot{J}_{a}^{\prime}(0,0) .
\end{array}\right.
$$

By the representation formula (2.9), we infer $\forall t$

$$
J_{a}^{\prime}(0, t)=-J_{0}(0, t) \int_{0}^{t} J_{1}^{*} R^{\prime} J_{a}(0, s) \mathrm{d} s+J_{1}(0, t) \int_{0}^{t} J_{0}^{*} R^{\prime} J_{a}(0, s) \mathrm{d} s .
$$

By Proposition B.3, we have

$$
R^{\prime}(0, t)=R^{\prime}(0,0)+t \dot{R}^{\prime}(0,0)+O\left(\varepsilon t^{2}\right) .
$$

Hence, we infer

$$
\begin{aligned}
J_{0}^{-1} J_{0}^{\prime}(0, t) & =-\int_{0}^{t} J_{1}^{*} R^{\prime} J_{a} \mathrm{~d} s+J_{0}^{-1} J_{1}(0, t) \int_{0}^{t} J_{0}^{*} R^{\prime} J_{a} \mathrm{~d} s \\
& =-\frac{t^{2}}{6} R^{\prime}(0,0)-\frac{t^{3}}{12} \dot{R}^{\prime}(0,0)+O\left(\varepsilon t^{4}+t^{6}\right) .
\end{aligned}
$$

With the same arguments, we get

$$
\begin{aligned}
J_{0}^{-1} J_{1}^{\prime}(0, t)=-\frac{t}{2} R^{\prime}(0,0) & -\frac{t^{2}}{6} \dot{R}^{\prime}(0,0)+O\left(\varepsilon t^{3}+t^{5}\right) . \\
& -401-
\end{aligned}
$$


On the other hand $S^{\prime}=-t J_{0}^{-1} J_{0}^{\prime} J_{0}^{-1} J_{1}+t J_{0}^{-1} J_{1}^{\prime}$. Together with (B.18) and (B.19), we prove (B.12). With the same arguments, we estimate on the axis

$$
\begin{aligned}
& J_{0}^{-1} \dot{J}_{0}^{\prime}(0, t)=-\frac{t}{2} R^{\prime}(0,0)-\frac{t^{2}}{3} \dot{R}^{\prime}(0,0)+O\left(\varepsilon t^{3}+t^{5}\right), \\
& J_{0}^{-1} \dot{J}_{1}^{\prime}(0, t)=-R^{\prime}(0,0)-\frac{t}{2} \dot{R}^{\prime}(0,0)+O\left(\varepsilon t^{2}+t^{4}\right),
\end{aligned}
$$

which yields (B.13).

It is obvious the $J_{a}^{\prime \prime}(0, t)$ for $a=1,2$ satisfies the following equations

$$
\left\{\begin{array}{l}
\ddot{J}_{a}^{\prime \prime}+R J_{a}^{\prime \prime}=-R^{\prime \prime} J_{a}-2 R^{\prime} J_{a}^{\prime}, \\
J_{a}^{\prime \prime}(0)=0=\dot{J}_{a}^{\prime \prime}(0) .
\end{array}\right.
$$

Applying the representation formula (2.9), we infer on the axis

$$
\begin{aligned}
J_{a}^{\prime \prime}(0, t)=-J_{0}(0, t) \int_{0}^{t} J_{1}^{*}( & \left.R^{\prime \prime} J_{a}+2 R^{\prime} J_{a}^{\prime}\right)(0, s) \mathrm{d} s \\
& +J_{1}(0, t) \int_{0}^{t}\left(R^{\prime \prime} J_{a}+2 R^{\prime} J_{a}^{\prime}\right)(0, s) \mathrm{d} s .
\end{aligned}
$$

By Proposition B.3, we have $R^{\prime \prime}(0, t)=R^{\prime \prime}(0)+t \dot{R}^{\prime \prime}(0)+O\left(\varepsilon t^{2}\right)$ so that

$$
\begin{aligned}
& J_{0}^{-1} J_{0}^{\prime \prime}(0, t) \\
& =-\int_{0}^{t} J_{1}^{*}\left(R^{\prime \prime} J_{a}+2 R^{\prime} J_{a}^{\prime}\right) \mathrm{d} s+\left(J_{0}^{-1} J_{1}\right)(0, t) \int_{0}^{t}\left(R^{\prime \prime} J_{a}+2 R^{\prime} J_{a}^{\prime}\right) \mathrm{d} s \\
& =-\frac{t^{2}}{6} R^{\prime \prime}(0,0)-\frac{t^{3}}{12} \dot{R}^{\prime \prime}(0,0)+O\left(\varepsilon t^{4}+t^{6}\right) .
\end{aligned}
$$

Similarly, we have

$$
J_{0}^{-1} J_{1}^{\prime \prime}(0, t)=-\frac{t}{2} R^{\prime \prime}(0,0)-\frac{t^{2}}{6} \dot{R}^{\prime \prime}(0,0)+O\left(\varepsilon t^{3}+t^{5}\right) .
$$

On the other hand, we remark $S^{\prime \prime}=2 t J_{0}^{-1} J_{0}^{\prime} J_{0}^{-1} J_{0}^{\prime} J_{1}-t J_{0}^{-1} J_{0}^{\prime \prime} J_{0}^{-1} J_{1}-$ $2 t J_{0}^{-1} J_{0}^{\prime} J_{0}^{-1} J_{1}^{\prime}+t J_{0}^{-1} J_{1}^{\prime \prime}$. Together with (B.23) and (B.24), we deduce (B.14). We finish the proof of Lemma B.5.

As consequences of Lemma B.5, the coefficients $a_{11}, a_{12}$ and $a_{12}$ in the $\mathcal{C}$-curvature (3.5) have the following expansion 
COROLlary B.7. - Under the same assumptions as in Lemma B.5, we have on the axis

$$
\begin{aligned}
& a_{11}\left(m_{0}, \nu_{0}, \xi\right)= R\left(\xi, E_{1}, \xi, E_{1}\right) \\
&+ \frac{3}{4} \tau\left(\nabla_{1} R_{1212} \xi_{2}^{2}+2 \nabla_{1} R_{1213} \xi_{2} \xi_{3}+\nabla_{1} R_{1313} \xi_{3}^{2}\right) \\
&+ \frac{2}{5} \tau^{2}\left(\xi_{2}^{2}+\xi_{3}^{2}\right)+O\left(\varepsilon \tau^{2}+\tau^{4}\right)\left(\xi_{2}^{2}+\xi_{3}^{2}\right) . \\
& a_{12}\left(m_{0}, \nu_{0}, \xi\right)=2 R\left(\xi, E_{1}, \xi, E_{2}\right)+\tau\left[\frac{1}{2} \nabla_{2} R_{1212} \xi_{2}^{2}+\left(\nabla_{1} R_{1223}+\nabla_{2} R_{1213}\right) \xi_{2} \xi_{3}\right. \\
&+\left(\nabla_{1} R_{1323}+\frac{1}{2} \nabla_{2} R_{1313}\right) \xi_{3}^{2} \\
&\left.\quad-\nabla_{1} R_{1212} \xi_{1} \xi_{2}-\nabla_{1} R_{1213} \xi_{1} \xi_{3}\right] \\
&-\frac{2}{5} \tau^{2} \xi_{1} \xi_{2}+O\left(\varepsilon \tau^{2}+\tau^{3}\right)\left(\xi_{2}^{2}+\xi_{3}^{2}+\xi_{1} \xi_{2}+\xi_{1} \xi_{3}\right) . \\
& a_{22}\left(m_{0}, \nu_{0}, \xi\right)=R(\xi,\left.E_{2}, \xi, E_{2}\right) \\
&+ \tau\left[\frac{1}{4} \nabla_{1} R_{1212} \xi_{1}^{2}-\frac{1}{2} \nabla_{2} R_{1212} \xi_{1} \xi_{2}\right. \\
& \quad-\frac{1}{2}\left(\nabla_{1} R_{1223}+\nabla_{2} R_{1213}\right) \xi_{1} \xi_{3}+\frac{1}{2} \nabla_{2} R_{1223} \xi_{2} \xi_{3} \\
&\left.+\left(\frac{1}{4} \nabla_{1} R_{2323}+\frac{1}{2} \nabla_{2} R_{1323}\right) \xi_{3}^{2}\right] \\
&+\frac{\tau^{2}}{15}\left(\xi_{1}^{2}+\xi_{2}^{2}+2 \xi_{3}^{2}\right)+O\left(\varepsilon \tau^{2}+\tau^{4}\right) .
\end{aligned}
$$

Proof. - We study first the coefficients $a_{11}$. In view of (B.11), we calculate

$$
\begin{aligned}
a_{11}\left(m_{0}, \nu_{0}, \xi\right)= & \langle R(0,0) \xi, \xi\rangle+\frac{3}{4} \tau\langle\dot{R}(0,0) \xi, \xi\rangle+\frac{2}{5} \tau^{2}\langle A \xi, \xi\rangle \\
& +O\left(\varepsilon \tau^{2}+\tau^{4}\right)\left(\xi_{2}^{2}+\xi_{3}^{2}\right) \\
= & R_{1212} \xi_{2}^{2}+2 R_{1213} \xi_{2} \xi_{3}+R_{1313} \xi_{3}^{2} \\
& +\frac{3}{4} \tau\left(\nabla_{1} R_{1212} \xi_{2}^{2}+2 \nabla_{1} R_{1213} \xi_{2} \xi_{3}+\nabla_{1} R_{1313} \xi_{3}^{2}\right) \\
& +\frac{2}{5} \tau^{2}\left(\xi_{2}^{2}+\xi_{3}^{2}\right)+O\left(\varepsilon \tau^{2}+\tau^{4}\right)\left(\xi_{2}^{2}+\xi_{3}^{2}\right)
\end{aligned}
$$




$$
\begin{aligned}
= & R\left(\xi, E_{1}, \xi, E_{1}\right) \\
& +\frac{3}{4} \tau\left(\nabla_{1} R_{1212} \xi_{2}^{2}+2 \nabla_{1} R_{1213} \xi_{2} \xi_{3}+\nabla_{1} R_{1313} \xi_{3}^{2}\right) \\
& +\frac{2}{5} \tau^{2}\left(\xi_{2}^{2}+\xi_{3}^{2}\right)+O\left(\varepsilon \tau^{2}+\tau^{4}\right)\left(\xi_{2}^{2}+\xi_{3}^{2}\right) .
\end{aligned}
$$

Therefore, we prove (B.25).

Now we calculate the coefficients $a_{12}$. Noting that $\langle\xi, P \xi\rangle=0$, thus the coefficient $a_{12}$ takes the form

$$
a_{12}\left(m_{0}, \nu_{0}, \xi\right)=-\frac{3}{\tau}\left\langle\dot{S}^{\prime} \xi, \xi\right\rangle-\frac{6}{\tau}\left\langle\dot{S} \xi, P^{\perp} \xi\right\rangle+\frac{3}{\tau^{2}}\left\langle S^{\prime} \xi, \xi\right\rangle+\frac{6}{\tau^{2}}\left\langle\left(S-I_{n}\right) \xi, P^{\perp} \xi\right\rangle .
$$

Plugging (B.9), (B.10), (B.12) and (B.13) into the above expression, we get

$$
\begin{aligned}
a_{12}\left(m_{0}, \nu_{0}, \xi\right)= & 2\left\langle R^{\prime}(0) \xi, \xi\right\rangle+\frac{3}{4} \tau\left\langle\dot{R}^{\prime}(0) \xi, \xi\right\rangle+4\left\langle R(0) \xi, P^{\perp} \xi\right\rangle \\
& +\frac{3}{2} \tau\left\langle\dot{R}(0) \xi, P^{\perp} \xi\right\rangle+\frac{8}{15} \tau^{2}\left\langle A \xi, P^{\perp} \xi\right\rangle-\left\langle R^{\prime}(0) \xi, \xi\right\rangle \\
& -\frac{\tau}{4}\left\langle\dot{R}^{\prime}(0) \xi, \xi\right\rangle-2\left\langle R(0) \xi, P^{\perp} \xi\right\rangle-\frac{\tau}{2}\left\langle\dot{R}(0) \xi, P^{\perp} \xi\right\rangle \\
& -\frac{2}{15} \tau^{2}\left\langle A \xi, P^{\perp} \xi\right\rangle+O\left(\varepsilon t^{2}+t^{4}\right)\left(\xi_{2}^{2}+\xi_{3}^{2}+\xi_{1} \xi_{2}+\xi_{1} \xi_{3}\right) \\
= & \left\langle R^{\prime}(0) \xi, \xi\right\rangle+2\left\langle R(0) \xi, P^{\perp} \xi\right\rangle \\
& +\tau\left(\frac{1}{2}\left\langle\dot{R}^{\prime}(0) \xi, \xi\right\rangle+\left\langle\dot{R}(0) \xi, P^{\perp} \xi\right\rangle\right) \\
& +\frac{2}{5} \tau^{2}\left\langle A \xi, P^{\perp} \xi\right\rangle+O\left(\varepsilon t^{2}+t^{4}\right)\left(\xi_{2}^{2}+\xi_{3}^{2}+\xi_{1} \xi_{2}+\xi_{1} \xi_{3}\right) .
\end{aligned}
$$

In view of Proposition B.3, we get

$$
\begin{aligned}
& a_{12}\left(m_{0}, \nu_{0}, \xi\right)=2 R_{1223} \xi_{2} \xi_{3}+2 R_{1323} \xi_{3}^{2}+2\left(-R_{1212} \xi_{1} \xi_{2}-R_{1213} \xi_{1} \xi_{3}\right) \\
&+ \tau\left[\frac{1}{2} \nabla_{2} R_{1212} \xi_{2}^{2}+\left(\nabla_{1} R_{1223}+\nabla_{2} R_{1213}\right) \xi_{2} \xi_{3}\right. \\
&+\left(\nabla_{1} R_{1323}+\frac{1}{2} \nabla_{2} R_{1313}\right) \xi_{3}^{2} \\
&\left.\quad-\nabla_{1} R_{1212} \xi_{1} \xi_{2}-\nabla_{1} R_{1213} \xi_{1} \xi_{3}\right] \\
&-\frac{2}{5} \tau^{2} \xi_{1} \xi_{2}+O\left(\varepsilon \tau^{2}+\tau^{3}\right)\left(\xi_{2}^{2}+\xi_{3}^{2}+\xi_{1} \xi_{2}+\xi_{1} \xi_{3}\right),
\end{aligned}
$$

which gives the desired result (B.26). Now we consider the coefficient $a_{22}$. We remark that $\left\langle P^{\perp} \xi, P^{\perp} \xi\right\rangle=\langle\xi, P \xi\rangle$ so that the coefficient $a_{22}$ takes the 
form

$$
\begin{aligned}
a_{22}\left(m_{0}, \nu_{0}, \xi\right)=-\frac{3}{2 \tau^{2}}\left\langle S^{\prime \prime} \xi, \xi\right\rangle & -\frac{3}{2 \tau}\langle\dot{S} \xi, \xi\rangle-\frac{6}{\tau^{2}}\left\langle S^{\prime} \xi, P^{\perp} \xi\right\rangle \\
& -\frac{3}{\tau^{2}}\left\langle\left(S-I_{n}\right) P^{\perp} \xi, P^{\perp} \xi\right\rangle+\frac{3}{\tau^{2}}\left\langle\left(S-I_{n}\right) \xi, P \xi\right\rangle .
\end{aligned}
$$

Plugging (B.9), (B.10), (B.12) and (B.14) into the above expression, we get

$$
\begin{aligned}
a_{22}\left(m_{0}, \nu_{0}, \xi\right)= & \frac{1}{2}\left\langle R^{\prime \prime}(0) \xi, \xi\right\rangle+\frac{\tau}{8}\left\langle\dot{R}^{\prime \prime}(0) \xi, \xi\right\rangle+\langle R(0) \xi, \xi\rangle+\frac{3 \tau}{8}\langle\dot{R}(0) \xi, \xi\rangle \\
& +\frac{2}{15} \tau^{2}\langle A \xi, \xi\rangle+2\left\langle R^{\prime}(0) \xi, P^{\perp} \xi\right\rangle+\frac{\tau}{2}\left\langle\dot{R}^{\prime}(0) \xi, P^{\perp} \xi\right\rangle \\
& +\left\langle R(0) P^{\perp} \xi, P^{\perp} \xi\right\rangle+\frac{\tau}{4}\left\langle\dot{R}(0) P^{\perp} \xi, P^{\perp} \xi\right\rangle+\frac{\tau^{2}}{15}\left\langle A P^{\perp} \xi, P^{\perp} \xi\right\rangle \\
& -\langle R(0) \xi, P \xi\rangle-\frac{\tau}{4}\langle\dot{R}(0) \xi, P \xi\rangle-\frac{\tau^{2}}{15}\langle A \xi, P \xi\rangle+O\left(\varepsilon \tau^{2}+\tau^{4}\right) \\
= & \frac{1}{2}\left\langle R^{\prime \prime}(0) \xi, \xi\right\rangle+2\left\langle R^{\prime}(0) \xi, P^{\perp} \xi\right\rangle+\langle R(0) \xi, \xi\rangle \\
& +\left\langle R(0) P^{\perp} \xi, P^{\perp} \xi\right\rangle-\langle R(0) \xi, P \xi\rangle \\
& +\tau\left[\frac{1}{8}\left\langle\dot{R}^{\prime \prime}(0) \xi, \xi\right\rangle+\frac{1}{2}\left\langle\dot{R}^{\prime}(0) \xi, P^{\perp} \xi\right\rangle+\frac{3}{8}\langle\dot{R}(0) \xi, \xi\rangle\right. \\
& \left.\quad+\frac{1}{4}\left\langle\dot{R}(0) P^{\perp} \xi, P^{\perp} \xi\right\rangle-\frac{1}{4}\langle\dot{R}(0) \xi, P \xi\rangle\right] \\
& +\frac{\tau^{2}}{15}\left[2\langle A \xi, \xi\rangle+\left\langle A P^{\perp} \xi, P^{\perp} \xi\right\rangle-\langle A \xi, P \xi\rangle\right]+O\left(\varepsilon \tau^{2}+\tau^{4}\right) .
\end{aligned}
$$

By Proposition B.3, we obtain

$$
\begin{aligned}
& a_{22}\left(m_{0}, \nu_{0}, \xi\right)=-R_{1213} \xi_{2} \xi_{3}+\left(-R_{1313}+R_{2323}\right) \xi_{3}^{2}-2 R_{1223} \xi_{1} \xi_{3}+R_{1212} \xi_{2}^{2} \\
& +2 R_{1213} \xi_{2} \xi_{3}+R_{1313} \xi_{3}^{2}+R_{1212} \xi_{1}^{2}-\left(R_{1212} \xi_{2}^{2}+R_{1213} \xi_{2} \xi_{3}\right) \\
& +\tau\left\{\frac { 1 } { 8 } \left[-\nabla_{1} R_{1212} \xi_{2}^{2}+4\left(-\nabla_{1} R_{1213}+\nabla_{2} R_{1223}\right) \xi_{2} \xi_{3}\right.\right. \\
& \left.+\left(2 \nabla_{1} R_{2323}-3 \nabla_{1} R_{1313}+4 \nabla_{2} R_{1323}\right) \xi_{3}^{2}\right] \\
& -\frac{1}{2}\left[\nabla_{2} R_{1212} \xi_{1} \xi_{2}+\left(\nabla_{1} R_{1223}+\nabla_{2} R_{1213}\right) \xi_{1} \xi_{3}\right] \\
& +\frac{3}{8}\left(\nabla_{1} R_{1212} \xi_{2}^{2}+2 \nabla_{1} R_{1213} \xi_{2} \xi_{3}+\nabla_{1} R_{1313} \xi_{3}^{2}\right) \\
& \left.+\frac{1}{4} \nabla_{1} R_{1212} \xi_{1}^{2}-\frac{1}{4}\left(\nabla_{1} R_{1212} \xi_{2}^{2}+\nabla_{1} R_{1213} \xi_{2} \xi_{3}\right)\right\} \\
& +\frac{\tau^{2}}{15}\left(2 \xi_{2}^{2}+2 \xi_{3}^{2}-\xi_{2}^{2}+\xi_{1}^{2}\right)+O\left(\varepsilon \tau^{2}+\tau^{4}\right)
\end{aligned}
$$




$$
\begin{aligned}
= & R_{1212} \xi_{1}^{2}-2 R_{1223} \xi_{1} \xi_{3}+R_{2323} \xi_{3}^{2} \\
+ & \tau\left[\frac{1}{4} \nabla_{1} R_{1212} \xi_{1}^{2}-\frac{1}{2} \nabla_{2} R_{1212} \xi_{1} \xi_{2}\right. \\
& \quad-\frac{1}{2}\left(\nabla_{1} R_{1223}+\nabla_{2} R_{1213}\right) \xi_{1} \xi_{3}+\frac{1}{2} \nabla_{2} R_{1223} \xi_{2} \xi_{3} \\
& \left.+\left(\frac{1}{4} \nabla_{1} R_{2323}+\frac{1}{2} \nabla_{2} R_{1323}\right) \xi_{3}^{2}\right] \\
+ & \frac{\tau^{2}}{15}\left(\xi_{1}^{2}+\xi_{2}^{2}+2 \xi_{3}^{2}\right)+O\left(\varepsilon \tau^{2}+\tau^{4}\right),
\end{aligned}
$$

which yields the desired (B.27). Therefore, we prove the result.

\section{B.2. The inverse of the Hessian of the squared distance near the focalization}

In this subsection we consider the approximation of the inverse of the squared distance and the associated derivatives.

Proposition B.8. - Let $(M, g)$ be a closed n-dimensional Riemannian manifold satisfying (1.3) and (1.4) (or (1.5)) with $\varepsilon$ small enough. Set $\left(m_{0}, \nu_{0}\right) \in$ NoCut, $\left|\nu_{0}\right| \geqslant \frac{3 \pi}{4}$. Let $x$ be the Fermi coordinate system associated to the geodesic $\exp _{m_{0}} t \nu_{0}$ for $t \in[0,1]$ and $v$ be the fiber coordinates of $T M \rightarrow M$ naturally associated to $x$. Then there exist positive numbers $\gamma_{1}, C_{8}>0$ such that for all $\varepsilon<\gamma_{1}$, we have

(1) $\left|S^{-1}\left(m_{0}, \nu_{0}, 1\right)-\bar{S}^{-1}\left(m_{0}, \nu_{0}, 1\right)\right| \leqslant C_{8} \varepsilon$;

(2) $\left|\partial_{x} S^{-1}\left(m_{0}, \nu_{0}, 1\right)-\partial_{x} \bar{S}^{-1}\left(m_{0}, \nu_{0}, 1\right)\right| \leqslant C_{8} \varepsilon$,

$\left|D_{v} S^{-1}\left(m_{0}, \nu_{0}, 1\right)-D_{v} \bar{S}^{-1}\left(m_{0}, \nu_{0}, 1\right)\right| \leqslant C_{8} \varepsilon ;$

(3) $\left|\partial_{x x}^{2} S^{-1}\left(m_{0}, \nu_{0}, 1\right)-\partial_{x x}^{2} \bar{S}^{-1}\left(m_{0}, \nu_{0}, 1\right)\right| \leqslant C_{8} \varepsilon$,

$\left|\partial_{x} D_{v} S^{-1}\left(m_{0}, \nu_{0}, 1\right)-\partial_{x} D_{v} \bar{S}^{-1}\left(m_{0}, \nu_{0}, 1\right)\right| \leqslant C_{8} \varepsilon$,

$\left|D_{v v}^{2} S^{-1}\left(m_{0}, \nu_{0}, 1\right)-D_{v v}^{2} \bar{S}^{-1}\left(m_{0}, \nu_{0}, 1\right)\right| \leqslant C_{8} \varepsilon$.

Proof. - Thanks to Lemma A.1 and Lemma A.2, $\partial_{x} X\left(m_{0}, \nu_{0}, 1\right)$ is inversible provided $\left|\nu_{0}\right| \geqslant \frac{3 \pi}{4}$ and $\varepsilon$ small enough. We state

$$
S^{-1}\left(m_{0}, \nu_{0}, 1\right)=\left(\partial_{x} X\left(m_{0}, \nu_{0}, 1\right)\right)^{-1} D_{v} X\left(m_{0}, \nu_{0}, 1\right)
$$

Thus, the desired results yield from Lemmas A.1, A.2, A.4, A.5, A.6 and A.7. Finally, we prove Proposition B.8. 


\section{B.3. Perturbative calculation of the Hessian of the squared dis- tance}

Proposition B.9. - Let $(M, g)$ be a closed n-dimensional Riemannian manifold satisfying (1.3) and (1.4) (or (1.5)) with $\varepsilon<\gamma$. Given any $\delta_{2} \in$ $(3 \pi / 4, \pi)$, let $\left(m_{0}, \nu_{0}\right) \in$ NoCut with $\tau=\left|\nu_{0}\right| \leqslant \delta_{2}$. Assume $\gamma$ is small enough such that $\delta_{2}<t_{F}\left(m_{0}, \nu_{0}\right)$ and

$$
\frac{\left|\nu_{0}\right|}{\sin \left|\nu_{0}\right|} \gamma \leqslant \frac{1}{4 \sqrt{n-1}} \text {. }
$$

Then there exists a positive constant $C \geqslant 1$ independent of $\left(m_{0}, \nu_{0}, \gamma\right)$ such that

$$
\begin{gathered}
|\ddot{S}-\ddot{\bar{S}}| \leqslant C \frac{\tau^{4}}{\sin ^{4} \tau} \gamma, \quad|\dot{S}-\dot{\bar{S}}| \leqslant C \frac{\tau^{4}}{\sin ^{3} \tau} \gamma, \quad|S-\bar{S}| \leqslant C \frac{\tau^{4}}{\sin ^{2} \tau} \gamma, \\
\left|S^{\prime}\right| \leqslant C \frac{\tau^{4}}{\sin ^{2} \tau} \gamma, \quad\left|\dot{S}^{\prime}\right| \leqslant C \frac{\tau^{4}}{\sin ^{3} \tau} \gamma, \quad\left|S^{\prime \prime}\right| \leqslant C \frac{\tau^{5}}{\sin ^{3} \tau} \gamma .
\end{gathered}
$$

Proof. - It is known that $[8$, Section 2$]$

$$
\begin{aligned}
\left|J_{0}^{-1}-\bar{J}_{0}^{-1}\right| & \leqslant 4 \sqrt{n-1}\left(\frac{\tau}{\sin \tau}\right)^{2} \gamma . \\
\left|J_{0}^{-1}\right| & \leqslant 2 \sqrt{n-1} \frac{\tau}{\sin \tau} .
\end{aligned}
$$

We adapt the proof in [8]. We investigate $S-\bar{S}$. From the expression of $\ddot{S}$, we have

$$
\begin{aligned}
\ddot{S}-\ddot{\bar{S}}= & 2\left(J_{0}^{-1}-\bar{J}_{0}^{-1}\right) \dot{J}_{1}+2 \bar{J}_{0}^{-1}\left(\dot{J}_{1}-\dot{\overline{J_{1}}}\right)-2 t\left(J_{0}^{-1}-\bar{J}_{0}^{-1}\right) \dot{J}_{0} J_{0}^{-1} \dot{J}_{1} \\
& -2 t \bar{J}_{0}^{-1}\left(\dot{J}_{0}-\dot{\bar{J}}_{0}\right) J_{0}^{-1} \dot{J}_{1}-2 t \bar{J}_{0}^{-1} \dot{\bar{J}}_{0}\left(J_{0}^{-1}-\bar{J}_{0}^{-1}\right) \dot{J}_{1} \\
& -2 t \bar{J}_{0}^{-1} \dot{\bar{J}}_{0} \bar{J}_{0}^{-1}\left(\dot{J}_{1}-\dot{\bar{J}}_{1}\right)+2 t\left(J_{0}^{-1}-\bar{J}_{0}^{-1}\right) \dot{J}_{0} J_{0}^{-1} \dot{J}_{0} J_{0}^{-1} J_{1} \\
& +2 t \bar{J}_{0}^{-1}\left(\dot{J}_{0}-\dot{\bar{J}}_{0}\right) J_{0}^{-1} \dot{J}_{0} J_{0}^{-1} J_{1}+2 t \bar{J}_{0}^{-1} \dot{\bar{J}}_{0}\left(J_{0}^{-1}-\bar{J}_{0}^{-1}\right) \dot{J}_{0} J_{0}^{-1} J_{1} \\
& +2 t \bar{J}_{0}^{-1} \dot{\bar{J}}_{0} \bar{J}_{0}^{-1}\left(\dot{J}_{0}-\dot{\bar{J}}_{0}\right) J_{0}^{-1} J_{1}+2 t \bar{J}_{0}^{-1} \dot{\bar{J}}_{0} \bar{J}_{0}^{-1} \dot{\bar{J}}_{0}\left(J_{0}^{-1}-\bar{J}_{0}^{-1}\right) J_{1} \\
& +2 t \bar{J}_{0}^{-1} \dot{\bar{J}}_{0} \bar{J}_{0}^{-1} \dot{\bar{J}}_{0} \bar{J}_{0}^{-1}\left(J_{1}-\bar{J}_{1}\right)-2 t\left(J_{0}^{-1}-\bar{J}_{0}^{-1}\right) \dot{J}_{0} J_{0}^{-1} \dot{J}_{1} \\
& -2 t \bar{J}_{0}^{-1}\left(\dot{J}_{0}-\dot{\bar{J}}_{0}\right) J_{0}^{-1} \dot{J}_{1}-2 t \bar{J}_{0}^{-1} \dot{\bar{J}}_{0}\left(J_{0}^{-1}-\bar{J}_{0}^{-1}\right) \dot{J}_{1} \\
& -2 t \bar{J}_{0}^{-1} \dot{\bar{J}}_{0} \bar{J}_{0}^{-1}\left(J_{1}-\bar{J}_{1}\right)+t\left(J_{0}^{-1}-\bar{J}_{0}^{-1}\right) \ddot{J}_{1}+t \bar{J}_{0}^{-1}\left(\ddot{J}_{1}-\ddot{\bar{J}}_{1}\right) .
\end{aligned}
$$

Together with (B.30), (B.31) and Lemmas A.1 and A.2, we infer the first estimate in (B.28). With the same arguments, we get the last two estimates in (B.28). On the other hand, using the representation formula (B.17) and (B.22) and Proposition B.3, we get $\left|J_{a}^{\prime}\right|=\mathcal{B}(\tau \gamma), \quad\left|J_{a}^{\prime \prime}\right|=\mathcal{B}(\tau \gamma)$, 
$\left|\dot{J}_{0}^{\prime}\right|=\mathcal{B}(\tau \gamma)$ for $a=0,1$. From the expression of $S^{\prime}, \dot{S}^{\prime}, S^{\prime \prime}$, we get the desired estimates in (B.29). Therefore, Proposition B.9 is proved.

\section{Bibliography}

[1] Y. Brenier, "Polar factorization and monotone rearrangement of vector-valued functions", Commun. Pure Appl. Math. 44 (1991), no. 4, p. 375-417.

[2] M. DO CARmo, Riemannian Geometry, Mathematics: Theory \& Applications, Birkhäuser, 1992.

[3] I. Chavel, Eigenvalues in Riemannian Geometry, Pure and Applied Mathematics, vol. 115, Academic Press Inc., 1984.

[4] J. Cheeger \& D. G. Ebin, Comparison Theorems in Riemannian Geometry, NorthHolland Mathematical Library, vol. 9, North-Holland, 1975.

[5] D. Cordero-Erausquin, "Sur le transport de mesures périodiques", C. R. Math. Acad. Sci. Paris 329 (1999), no. 3, p. 199-202.

[6] P. Delanoë, private communication.

[7] - "On the smoothness of the potential function in Riemannian optimal transport", Commun. Anal. Geom. 23 (2015), no. 1, p. 11-89.

[8] P. Delanö̈ \& Y. Ge, "Regularity of optimal transportation maps on compact, locally nearly spherical, manifolds", J. Reine Angew. Math. 646 (2010), p. 65-115.

[9] — , "Locally nearly spherical surfaces are almost-positively curved", Methods Appl. Anal. 18 (2011), p. 269-302.

[10] P. Delanoë \& F. Rouvière, "Positively curved riemannian locally symmetric spaces are positively squared distance curved", Can. J. Math. 65 (2013), no. 4, p. 757-767.

[11] A. Figalli, "Existence, uniqueness, and regularity of optimal transport maps", SIAM J. Math. Anal. 39 (2007), no. 1, p. 126-137.

[12] A. Figalli, Y.-H. Kim \& R. J. MCCANN, "Hölder continuity and injectivity of optimal transport maps", Arch. Ration. Mech. Anal. 209 (2013), p. 747-795.

[13] - "Regularity of optimal transport maps on multiple products of spheres", $J$. Eur. Math. Soc. 15 (2013), no. 4, p. 1131-1166.

[14] A. Figalli \& L. Rifford, "Continuity of optimal transport maps on small deformations of $\mathbb{S}^{2} "$, Commun. Pure Appl. Math. 62 (2009), no. 12, p. 1670-1706.

[15] A. Figalli, L. Rifford \& C. Villani, "On the Ma-Trudinger-Wang curvature on surfaces", Calc. Var. Partial Differ. Equ. 39 (2010), no. 3-4, p. 307-332.

[16] - "Necessary and sufficient conditions for continuity of optimal transport maps on Riemannian manifolds", Tôhoku Math. J. 63 (2011), p. 855-876.

[17] — "Nearly round spheres look convex", Am. J. Math. 134 (2012), no. 1, p. 109139.

[18] S. Gallot, D. Hulin \& J. Lafontaine, Riemannian Geometry, 2nd ed., Universitext, Springer, 1990.

[19] D. Gilbarg \& N. S. Trudinger, Elliptic Partial Differential Equations of Second Order, Grundlehren der Mathematischen Wissenschaften, vol. 224, Springer, 1977.

[20] L. V. Kantorovich, "On a problem of Monge", Usp. Mat. Nauk 3 (2006), no. 2, p. 225-226, English translation in J. Math. Sci., New York 133, no. 4, p. 13-83.

[21] Y.-H. Kim, "Counterexamples to continuity of optimal transportation on positively curved Riemannian manifolds", Int. Math. Res. Not. 2008 (2008), article no. rnn120 (15 pages).

[22] Y.-H. Kim \& R. J. MCCANN, "Continuity, curvature, and the general covariance of optimal transportation", J. Eur. Math. Soc. 12 (2010), no. 4, p. 1009-1040. 
[23] - "Towards the smoothness of optimal maps on Riemannian submersions and Riemannian products (of round spheres in particular)", J. Reine Angew. Math. 664 (2012), p. 1-27.

[24] P. W. Y. LeE, "New computable necessary conditions for the regularity theory of optimal transportation", SIAM J. Math. Anal. 42 (2010), no. 6, p. 3054-3075.

[25] P. W. Y. LeE \& J. Li, "New examples satisfying Ma-Trudinger-Wang conditions", SIAM J. Math. Anal. 44 (2012), no. 1, p. 61-73.

[26] J. LıU, "Hölder regularity of optimal mappings in optimal transportation", Calc. Var. Partial Differ. Equ. 34 (2009), no. 4, p. 435-451.

[27] J. Liu, N. S. Trudinger \& X.-J. Wang, "Interior $C^{2, \alpha}$ regularity for potential functions in optimal transportation", Commun. Partial Differ. Equations 35 (2010), no. 1, p. $165-184$.

[28] G. Loeper, "On the regularity of solutions of optimal transportation problems", Acta Math. 202 (2009), no. 2, p. 241-283.

[29] - "Regularity of optimal maps on the sphere: The quadratic cost and the reflector antenna", Arch. Ration. Mech. Anal. 199 (2011), no. 1, p. 269-289.

[30] G. Loeper \& C. Villani, "Regularity of optimal transport in curved geometry: the nonfocal case", Duke Math. J. 151 (2010), no. 3, p. 431-485.

[31] X.-N. Ma, N. S. Trudinger \& X.-J. Wang, "Regularity of potential functions of the optimal transportation problem", Arch. Ration. Mech. Anal. 177 (2005), no. 2, p. 151-183.

[32] R. J. McCann, "Polar factorization of maps on Riemannian manifolds", Geom. Funct. Anal. 11 (2001), no. 3, p. 589-608.

[33] W. Meyer, Toponogov's Theorem and Applications, Lecture Notes, Trieste, 1989.

[34] G. Monge, Mémoire sur la théorie des déblais et remblais, Mémoires de l'Académie Royale des Sciences de Paris, 1781.

[35] N. S. Trudinger \& X.-J. WANG, "On the second boundary value problem for MongeAmpère type equations and optimal transportation", Ann. Sc. Norm. Super. Pisa, Cl. Sci. 8 (2009), no. 1, p. 143-174.

[36] C. Villani, Optimal transport, old and new, Grundlehren der Mathematischen Wissenschaften, vol. 338, Springer, 2009. 